\title{
Topological Invariants of Principal $G$-bundles with Singularities
}

Fabián Antonio Arias Amaya

Thesis submitted to the Departamento de Matemáticas

for the degree of $\mathrm{PhD}$ in Mathematics

Advisor:

Mikhail Malakhaltsev

Universidad de Los Andes

Facultad de Ciencias

Departamento de Matemáticas

Bogotá

2016 



\section{Topological Invariants of Principal $G$-bundles with Singularities}





\section{Dedicatory}

This thesis is dedicated to my wife and my children for their long wait during my absence, and my parents and brothers for their great support at all time. 


\section{Acknowledgement}

I thank ...

God for having joined my family all the time,

my advisor for his great support during the development of this work,

Universidad de los Andes, and specially the Departamento de Matemáticas for the financial support and its teaching,

Universidad Tecnólogica de Bolívar for the financial support. 


\section{Abstract}

Arias Amaya, F. A. Topological Invariants of Principal G-bundles with Singularities. 2016. 88 pages. Thesis of doctorate. Departamento de Matemáticas, Universidad de los Andes, Bogotá Colombia, 2016.

In this work we present an introduction to the theory of principal bundles with singularities, i.e. principal bundles which reduce to a closed subgroup of the structure group outside of a closed subset of the base space.

In the first part we give the definition of these new structures, and we define morphisms between them. Then, we prove that these structures and their morphisms form a category which contains the bundles induced by transversal maps, and we use the obstruction theory to construct characteristic class of principal bundles with singularities.

In the second part we present a version of Gauss-Bonnet-Hopf-Poincaré Formula for locally trivial fiber bundles over 2-dimensional manifolds, and we prove that this result generalizes the classical Gauss-Bonnet theorem. Then, we define branched sections of locally trivial fiber bundles, index of a singular point of a branched section, and give examples of its calculation, in particular for branched sections defined by binary differential equations. We also define a resolution of singularities of a branched section, and prove an analog of Gauss-Bonnet-Hopf-Poincaré formula for the branched sections admitting a resolution in case the manifold $M$ has dimension two.

Keywords: Principal bundles with singularities, obstruction theory, branched section, index of singular point, binary differential equations, curvature. 


\section{Contents}

$\begin{array}{ll}\text { Notations } & \text { ix }\end{array}$

$\begin{array}{lc}\text { Introduction } & \mathbf{x i}\end{array}$

1 Preliminaries. 1

1.1 Homotopy Groups. . . . . . . . . . . . . . . . . . . . . . . . . . . . 1

1.1.1 Definition and examples . . . . . . . . . . . . . . . 1

1.1.2 Action of $\pi_{1}\left(X, x_{0}\right)$ on $\pi_{n}\left(X, x_{0}\right) \ldots \ldots \ldots \ldots$. . . . . . . . . 2

$1.2 \mathrm{CW}$-Complexes . . . . . . . . . . . . . . . . . . . . 2

1.2.1 Homology and Cohomology of $C W$-complexes. . . . . . . . . . . . . . . . 4

1.3 Fiber Bundles . . . . . . . . . . . . . . . . . . . . . . . . . 7

1.3.1 Principal Bundles. . . . . . . . . . . . . . . . . . . . . 9

1.3.2 Associated and induced bundles. . . . . . . . . . . . . . . . . . . 11

1.4 The Bundle of Linear Frames. . . . . . . . . . . . . . . . . . . . . . . . . . . . 12

1.5 The extension problem. . . . . . . . . . . . . . . . . . . 13

1.5.1 The extension problem for maps. . . . . . . . . . . . . . . . . 14

1.5.2 The extension problem for sections of fiber bundles. . . . . . . . . . . . 16

2 Principal G-Bundles with Singularities. $\quad 21$

2.1 The Category of Principal G-bundles with singularities. . . . . . . . . . . . . . . . 21

2.2 Singularities and Indexes. . . . . . . . . . . . . . . . . . . . . 24

2.2.1 Isolated Singularities and Indexes. . . . . . . . . . . . . . . . . . 24

2.2.2 Singularities of higher dimension and indexes. . . . . . . . . . . . . 26

2.2.3 Characteristic classes of principal $G$-bundles with singularities. . . . . . . . . . 28

3 A Generalization of Gauss-Bonnet-Hopf-Poincaré Formula 31 
3.1 Singularity of section and its index $\ldots \ldots \ldots \ldots \ldots$

3.1.1 Sections with singularities. Resolution of singularity . . . . . . . . . . . . . 31

3.1 .2 Index of a singular point $x \in \Sigma \ldots \ldots \ldots \ldots \ldots \ldots$

3.2 Connection and the Gauss-Bonnet theorem . . . . . . . . . . . . . . . . . . 36

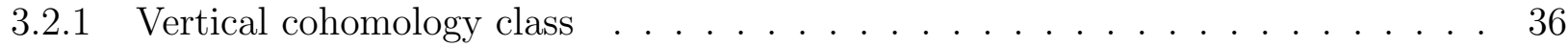

3.2 .2 Linear connection in the vertical bundle $V \rightarrow E \ldots \ldots \ldots$. . . . . . 37

3.2 .3 Structure equations of connection . . . . . . . . . . . . . . 38

3.2.4 Generalization of the Gauss-Bonnet-Hopf-Poincaré Formula . . . . . . . . . 39

3.3 Examples . . . . . . . . . . . . . . . . . . . . . . . . 41

3.3.1 Classical Gauss-Bonnet-Hopf-Poincaré Formula . . . . . . . . . . . . . . . . 41

3.3.2 Gauss-Bonnet-Hopf-Poincaré Formula for Projective Bundles . . . . . . . . . . 44

3.3.3 Section of projective bundle and reduction of the bundle of projective frames . 48

3.4 Gauss-Bonnet-Hopf-Poincaré formula in $G$-bundles with Singularities . . . . . . . . 51

3.4.1 The vertical subbundle of an associated fiber bundle . . . . . . . . . . . . 52

3.4.2 The Gauss-Bonnet-Hopf-Poincaré formula for principal G-bundle with singu-

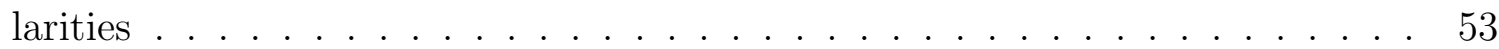

3.4.3 Hopf-Gauss-Bonnet-Hopf-Poincaré formula for the case of the sum of Whitney of vector bundles . . . . . . . . . . . . . . . . . . . 55

3.5 The Gauss-Bonnet-Hopf-Poincaré formula to branched sections. . . . . . . . . . . . . 57

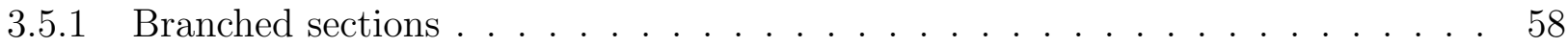

3.6 The index of a singular isolated point $\ldots \ldots \ldots \ldots \ldots$

3.6 .1 Local monodromy group . . . . . . . . . . . . . . . . . . . 60

3.6.2 The index of isolated singular point . . . . . . . . . . . . . . . 62

3.7 Resolution of a branched section . . . . . . . . . . . . . . . . . . . . . . . . 65

3.8 Connection and the Gauss-Bonnet theorem . . . . . . . . . . . . . . . . . . . . 69 


\section{Notations}

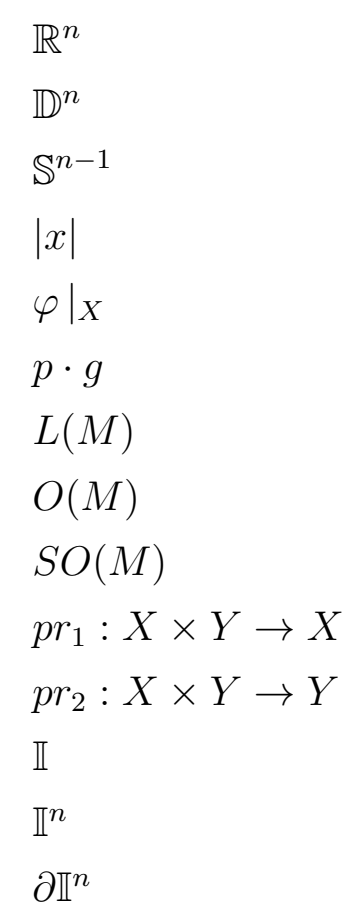

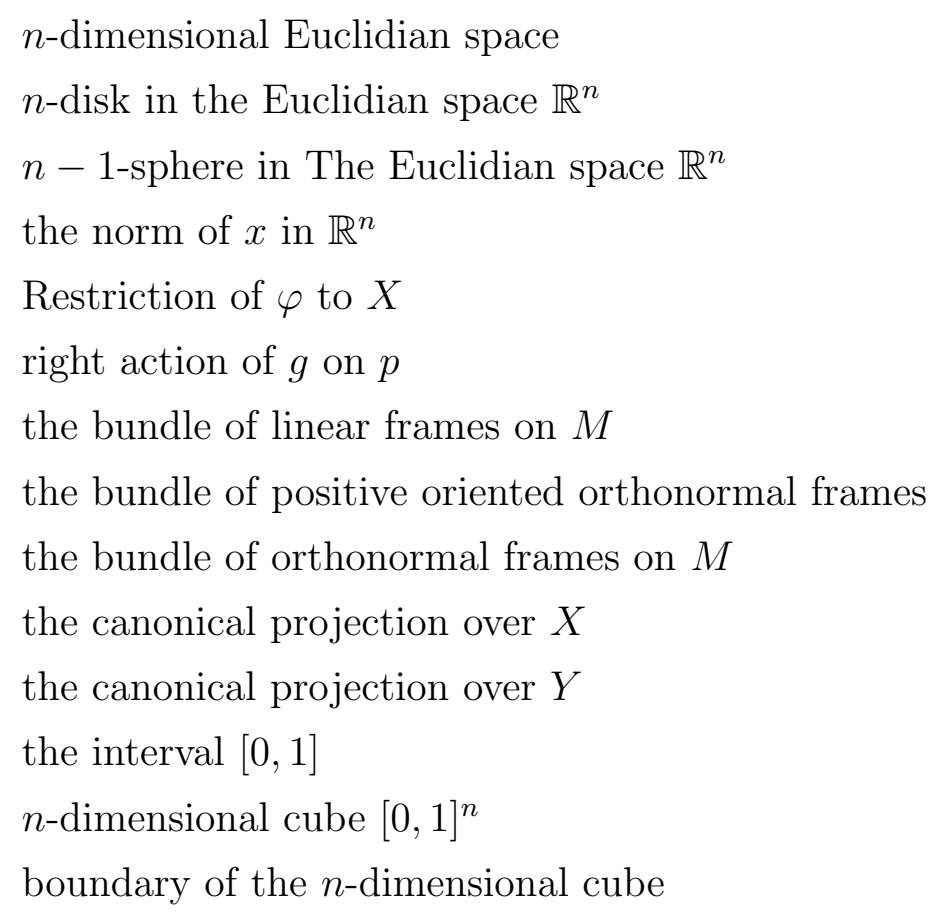

We assume all the manifolds to be smooth, and all the maps between manifolds to be smooth, as well. 


\section{Introduction.}

The classical theory of $G$-structures deals with regular geometrical structures, this means there hold some nondegeneracy conditions, as for example for Riemannian or symplectic structures. However when considering various geometrical problems there naturally arise "singular points", where the regularity conditions fail to be true. For example, a vector field can have zeros, a symplectic form $\omega$ restricted to a submanifold has points where $\operatorname{det}(\omega)=0$, the lift of a Riemannian metric to the bundle of orthonormal frames degenerates at some points, the curvature lines of a surface are not defined in umbilic points, etc., therefore it is natural to study the singularities of geometrical structures and their differential and topological invariants.

In terms of principal bundles, a singularity of a geometrical structure can be described as follows. Assume that $\bar{P} \rightarrow M$ is a $\bar{G}$-structure and $\Sigma \subset M$ is a subset. We say that this bundle is a $G$ structure with singularities $\Sigma$ if $\bar{P}$ reduces to a $G$-principal subbundle, where $G \subset \bar{G}$, over $M \backslash \Sigma$. For example, if $V$ is a vector field on a two-dimensional oriented Riemannian manifold $M$, and $\Sigma$ is the set of zeros of $V$, then the bundle $S O(M)$ of the positively oriented orthonormal frames of $M$ reduces to the trivial subgroup $\{e\} \subset S O(2)$ over $M \backslash \Sigma$. Another natural example is that, if $M$ is a surface in the three-dimensional Euclidean space, the bundle $S O(M)$ reduces to a discrete subgroup over $M \backslash \Sigma$, where $\Sigma$ is the set of umbilic points of $M$.

In this thesis we find topological invariants of singularities of principal bundles and prove some analogs of the Gauss-Bonnet and the Hopf-Poincaré theorems.

The thesis consists of three chapters. In the first chapter we give a brief description of the main definitions and results used in the work. This chapter begins with the definition of the homotopy groups, then we introduce an action of the fundamental group on the higher homotopy groups which allows us to give a definition of simple. The next section deals with $C W$-complexes, cohomology of $C W$-complexes and the extension problem for sections of locally trivial fiber bundles. After that, we give a brief review of principal bundles. At the end we provide a review of the obstruction theory.

In the second chapter we give the definitions of principal $G$-bundles with singularities and mor- 
phisms between them, and show that these objects and morphisms form a category. This chapter also contains a definition of characteristic classes of principal $G$-bundles with singularities. After that, we construct characteristic classes of these structures which allow us to associate an index to each singular point. The definitions and constructions are illustrated with examples. The main result of this chapter is Theorem 2.2.7.

In the third chapter we find a generalization of the Gauss-Bonnet-Hopf-Poincaré formula for sections with singularities of locally trivial bundles, and we prove that this result reduces to the classical one, if the fiber bundle is the tangent bundle of an oriented surface. Then we apply this result to principal bundles with singularities. The chapter finishes with a generalization of the previous result to branched sections of locally trivial fiber bundles, in particular to the branched section determined by a binary differential form. The main results of this chapter are Theorem 3.2.7 and Theorem 3.8.2.

The results of this work we reported at the following scientific events:

1. Congreso Colombiano de Matemáticas, Manizales, Colombia, 2015.

2. XI Encuentro Internacional de Matemáticas, Barranquilla, 2015.

3. Congreso Latinoamericano de Matemáticas, Barranquilla, 2016.

The results of the thesis were partially published in [10], [11]. Also the ideas close to the main lines of the work were used to define topological index of a singular point of 3-web in [12] 


\section{Chapter 1}

\section{Preliminaries.}

\subsection{Homotopy Groups.}

This section contains the results on homotopy theory which will use throughout the work.

\subsubsection{Definition and examples}

In this section we will consider topological spaces and continuous maps. For details we refer the reader to $[21],[1]$.

Definition 1.1.1. Let $X$ and $Y$ be topological spaces, $A \subset X$, and let $f: X \rightarrow Y$ and $g: X \rightarrow Y$ be two continuous maps. We say that $f$ and $g$ are homotopic relative to $A$ if $\left.f\right|_{A}=\left.g\right|_{A}$, and there exists a continuous map $H: X \times I \rightarrow Y$ such that $H(x, 0)=f, H(x, 1)=g$, and $\left.f\right|_{A}=H(., t)=\left.g\right|_{A}$ for all $t \in I$.

The relative homotopy is an equivalence relation in the set $[(X, A),(Y, B)]$ of continuous maps $f: X \rightarrow Y$ such that $f(A) \subset B$, and its equivalence classes are called relative homotopy classes. By $[f]$ we denote the relative homotopy class of $f$, and by $\pi_{n}\left(X, x_{0}\right)$, the set $\left[\left(\mathbb{I}^{n}, \partial \mathbb{I}^{n}\right),\left(X, x_{0}\right)\right]$.

The set $\pi_{n}\left(X, x_{0}\right)$ is endowed by a binary operation defined as follows: For $[f],[g] \in \pi_{n}\left(X, x_{0}\right)$, we set $[f] \cdot[g]=[f \cdot g]$, where the map $f \cdot g \in\left[\left(\mathbb{I}^{n}, \partial \mathbb{I}^{n}\right),\left(X, x_{0}\right)\right]$ is given by

$$
(f \cdot g)\left(t_{1}, \cdots, t_{n}\right)= \begin{cases}f\left(2 t_{1}, t_{2}, \cdots, t_{n}\right) & \text { if } 0 \leq t_{1} \leq 1 / 2 \\ g\left(2 t_{1}-1, t_{2}, \cdots, t_{n}\right) & \text { if } 1 / 2 \leq t_{1} \leq 1\end{cases}
$$

Proposition 1.1.1 ([1], page 340). The pair $\left(\pi_{n}\left(X, x_{0}\right), \cdot\right)$ is a group which is commutative for $n>1$. 


\subsubsection{Action of $\pi_{1}\left(X, x_{0}\right)$ on $\pi_{n}\left(X, x_{0}\right)$.}

Since there exists a canonical homeomorphism between the pairs $\left(\mathbb{D}^{n}, \mathbb{S}^{n-1}\right)$ and $\left(\mathbb{I}^{n}, \partial \mathbb{I}^{n}\right)$, the group $\pi_{n}\left(X, x_{0}\right)$ can be interpreted as the set of homotopy classes relative to $\mathbb{S}^{n-1}$ of maps $\left(\mathbb{D}^{n}, \mathbb{S}^{n-1}\right) \rightarrow$ $\left(X, x_{0}\right)$. By using this interpretation, now we define an action of $\pi_{1}\left(X, x_{0}\right)$ on $\pi_{n}\left(X, x_{0}\right)$.

Let $X$ be a path connected topological space, and $x_{0} \in X$. For $[\gamma] \in \pi_{1}\left(X, x_{0}\right)$, we define a map $\gamma^{*}: \pi_{n}\left(X, x_{0}\right) \rightarrow \pi_{n}\left(X, x_{0}\right)$ as follows. For $[f] \in \pi_{n}\left(X, x_{0}\right)$ we set $\gamma^{*} f: \mathbb{D}^{n} \rightarrow X$ by

$$
\left(\gamma^{*} f\right)(u)= \begin{cases}f(2 u) & \text { if } 0 \leq|u| \leq 1 / 2 \\ \gamma(2-2|u|), & \text { if } 1 / 2 \leq|u| \leq 1,\end{cases}
$$

where $|u|$ denotes the norm of $u$ in $\mathbb{R}^{n+1}$.

The homotopy class $\left[\gamma^{*} f\right]$ is independent of the representatives of $[\gamma]$ and $[f]$. Moreover, for all $[\gamma]$ and $[\eta]$ in $\pi_{1}\left(X, x_{0}\right)$, and $[f] \in \pi_{n}\left(X, x_{0}\right)$ we have

$$
\left[x_{0}^{*} f\right]=[f] \text {, and }\left[(\gamma \cdot \eta)^{*} f\right]=\left[\gamma^{*}\left(\eta^{*} f\right)\right],
$$

where $\left[x_{0}\right]$ represents the identity element of $\pi_{1}\left(X, x_{0}\right)$. Then the map $\pi_{1}\left(X, x_{0}\right) \times \pi_{n}\left(X, x_{0}\right) \rightarrow$ $\pi_{n}\left(X, x_{0}\right)$ given by $([\gamma],[f]) \mapsto\left[\gamma^{*} f\right]$ defines an action of $\pi_{1}\left(X, x_{0}\right)$ on the set $\pi_{n}\left(X, x_{0}\right)$.

Remark 1.1.1. If the action of $\pi_{1}\left(X, x_{0}\right)$ on $\pi_{n}\left(X, x_{0}\right)$ is trivial, we call $X$ an $n$-simple space. When a space $X$ is $n$-simple, each map $\mathbb{S}^{n} \longrightarrow X$ determines an element of $\pi_{n}\left(X, x_{0}\right)$. If a space $X$ is $n$-simple for all $n, X$ is called a simple space. It follows from the definition of this action that if $X$ is a simple space, then $\pi_{1}(X)$ is abelian.

When the topological space $X$ is a Lie group, or a quotient of Lie groups, we have the following results about their homotopy groups.

Theorem 1.1.2 ([21], 16.9). Let $G$ be a Lie group. Then $\pi_{1}(G)$ acts trivially on $\pi_{n}(G)$ for all $n \geq 0$. Therefore, $G$ is a simple space.

Proposition 1.1.3 ([21], 16.11). If $\bar{G}$ is a Lie group, and $G$ is a closed connected Lie subgroup, then $\bar{G} / G$ is simple. Hence, for any $n$, a map $\mathbb{S}^{n} \rightarrow \bar{G} / G$ represents an element of $\pi_{n}(\bar{G} / G)$.

\subsection{CW-Complexes}

In this section we present the definition of $C W$-complex, and some results concerning $C W$-complexes.

Definition 1.2.1. Let $X$ be a topological space. A $q$-cell in $X$ is a continuous map $e^{q}: \mathbb{D}^{q} \rightarrow X$ such that $\left.e^{q}\right|_{\left(\mathbb{D}^{q} \backslash \mathbb{S}^{q-1}\right)}$ is a homeomorphism onto its image. The map $\partial e^{q}=\left.e^{q}\right|_{\mathbb{S}^{q-1}}: \mathbb{S}^{q-1} \rightarrow X$ is called the boundary of $e^{q}$. 
Definition 1.2.2. Let $X$ be a topological space and let $A \subset X$ be a closed subspace. We say $X$ is obtained from $A$ by adjoining $q$-cells $\left\{e_{i}^{q} \mid i \in I\right\}$ in $X$ if the following conditions hold true:

1. $\partial e_{i}^{q}\left(\mathbb{S}^{q-1}\right), i \in I$ is contained in $A$ and $e_{i}^{q}\left(\mathbb{D}^{q} \backslash \mathbb{S}^{q-1}\right) \cap e_{j}^{q}\left(\mathbb{D}^{q} \backslash \mathbb{S}^{q-1}\right), i \neq j$ is empty.

2. A subset $B \subset X$ is closed if and only if $B \cap A$ closed in $A$ and $B \cap e_{i}^{q}\left(\mathbb{D}^{q} \backslash \mathbb{S}^{q-1}\right)$ is closed in $e_{i}^{q}\left(\mathbb{D}^{q}\right)$ for all $q$.

3. $X=A \cup_{i} e_{i}^{q}\left(\mathbb{D}^{q} \backslash \mathbb{S}^{q-1}\right)$.

Definition 1.2.3. Let $X$ be a topological space and $A$ be a closed subspace of $X$. The pair $(X, A)$ is called a relative $C W$-complex if there is a sequence of closed topological subspaces $\left\{X_{q}\right\}_{q \geq-1}$ which satisfy the following properties:

1. $X_{-1}=A$ and $X_{q+1}$ is obtained from $X_{q}$ by adjoining $(q+1)$-cells,

2. $X=\cup_{q \geq-1} X_{q}$.

3. $B \subset X$ is closed in $X$ if and only if $B \cap X_{q}$ is closed in $X_{q}$ for all $q$.

The relative $C W$-complex $(X, \phi)$ is called a $C W$-complex. If $X$ is a $C W$-complex and $X=X_{q}$ for some $q$, we call $q$ the dimension of $X$. A $C W$-complex is called a finite if has only finitely many cells. A subcomplex of a $C W$-complex $X$ is a subspace $A \subset X$ which is a union of cells of $X$. Moreover, we say that the relative $C W$-complex $(X, A)$ is a $C W$-pair if $A$ is a $C W$-subcomplex of $X$.

Remark 1.2.1. Let $B$ and $F$ be finite $C W$-complexes, then the product $B \times F$ is a $C W$-complex. Indeed, if $e^{k}: \mathbb{D}^{k} \rightarrow B$ is a $k$-cell in $B$, and $e^{l}$ is a $l$-cell in $F$, then the product $\mathbb{D}^{k} \times \mathbb{D}^{l} \rightarrow B \times F$ of these maps gives a $(k+l)$-cell $\mathbb{D}^{k+l} \rightarrow B \times F$ in $B \times F$ which we denote by $e^{k} \times e^{l}$.

Proposition 1.2.1. Let $E, B$ and $F C W$-complexes, and let $\pi: E \rightarrow B$ be a fiber bundle with typical fiber $F$. Then $E$ admits a $C W$-complex structure with cells $e^{k} \times e^{l}$, where $e^{k}$ and $e^{l}$ are cells on $B$ and $F$, respectively.

Proof. Let $e^{k}: \mathbb{D}^{k} \rightarrow B$ be a $k$-cell in $B$. Since $\mathbb{D}^{k}$ is contractible, the induced bundle $\left(e^{k}\right)^{*} E \rightarrow \mathbb{D}^{k}$ is trivial. We choose a trivialization $\varphi_{k}: \mathbb{D}^{k} \times F \rightarrow\left(e^{k}\right)^{*} E$ and consider the following commutative diagram

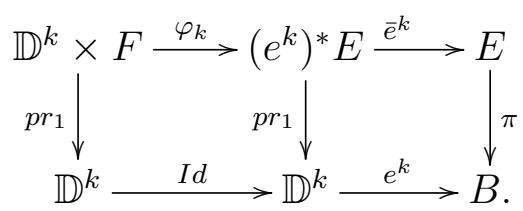

where $\bar{e}^{k}:\left(e^{k}\right)^{*} E \rightarrow E$ is the projection over $E$. Since $\left.e^{k}\right|_{\text {int }\left(\mathbb{D}^{k}\right)}$ is a homeomorphism onto its image, and the restriction $\left.\bar{e}^{k}\right|_{p r_{1}^{-1}\left(\operatorname{int}\left(\mathbb{D}^{k}\right)\right)}$ is also a homeomorphism, for any $l$-cell $e^{l}: \mathbb{D}^{l} \rightarrow F$ of $F$ we obtain 
a map $e^{k+l}: \mathbb{D}^{k} \times \mathbb{D}^{l} \rightarrow E$ given by the composition $\mathbb{D}^{k} \times \mathbb{D}^{l} \stackrel{\left(i d, e^{l}\right)}{\rightarrow} \mathbb{D}^{k} \times F \stackrel{\varphi_{k}}{\rightarrow}\left(e^{k}\right)^{*} E \stackrel{\bar{e}^{k}}{\rightarrow} E$ whose restriction to $\operatorname{int}\left(\mathbb{D}^{k}\right) \times \operatorname{int}\left(\mathbb{D}^{l}\right)$ is a homeomorphism. Therefore $e^{k+l}$ is a $(k+l)$-cell of $E$. We denote it by $e^{k} \times e^{l}$. Now, we show that the collection $\left\{e^{k} \times e^{l}: e^{k}\right.$ is a cell in $B, e^{l}$ is a cell in $\left.F\right\}$ of all $(k+l)$-cells constructed in this way gives a CW-decomposition of $E$. To do this, it is sufficient to show that $E=\cup_{k, l}\left(e^{k} \times e^{l}\right)\left(\operatorname{int}\left(\mathbb{D}^{k}\right) \times \operatorname{int}\left(\mathbb{D}^{l}\right)\right)$. Let $x \in E$, then $\pi(x)$ belongs to the interior of a unique cell in $B$, so there exists a unique $y \in \operatorname{int}\left(\mathbb{D}^{t}\right)$ for some non-negative integer $t$ such that $e^{t}(y)=\pi(x)$. Moreover, since $\bar{e}^{t} \circ \varphi_{t}: \mathbb{D}^{t} \times F \rightarrow E$ is a homeomorphism over $\operatorname{int}\left(\mathbb{D}^{t} \times F\right)$, there exists a unique $z$ in the interior of some cell of $F$ such that $\bar{e}^{t} \circ \varphi_{t}(y, z)=x$. Therefore, there exists a nonnegative integer $s$ and $u \in \mathbb{D}^{s}$ such that $e^{t} \times e^{s}(y, z)=x$. Therefore, $x \in\left(e^{t} \times e^{s}\right)\left(\operatorname{int}\left(\mathbb{D}^{t}\right) \times \operatorname{int}\left(\mathbb{D}^{s}\right)\right)$. Finally, we need to prove that $\partial\left(e^{k} \times e^{l}\right) \subset E^{k+l-1}$, and to do this, it is enough to note that $\partial\left(\mathbb{D}^{k} \times \mathbb{D}^{l}\right)=\partial \mathbb{D}^{k} \times \mathbb{D}^{l} \cup \mathbb{D}^{k} \times \partial \mathbb{D}^{l}$.

Definition 1.2.4. A continuous map $f: X \rightarrow Y$ between $C W$-complexes $X$ and $Y$ is called a cellular map if $f$ takes each $k$-skeleton $X^{k}$ of $X$ onto the $k$-skeleton $Y^{k}$ of $Y$ for each $k$.

Theorem 1.2.2 ([3], page 44). Every continuous map between $C W$-complexes is homotopic to a cellular map.

\subsubsection{Homology and Cohomology of $C W$-complexes.}

In this section we present the definition and results concerning the homology and cohomology of $C W$-complexes. For details we refer the reader to [1] and [16].

\section{Singular homology.}

Definition 1.2.5. The standard $q$-simplex $\Delta^{q}$ is defined by

$$
\Delta^{q}=\left\{\left(t_{0}, \cdots, t_{q}\right) \in \mathbb{R}^{q+1}: t_{i} \geq 0, \sum_{i=0}^{q} t_{i}=1\right\}
$$

The face maps are the functions $f_{q}^{m}: \Delta^{q} \rightarrow \Delta^{q+1}$ defined by

$$
f_{q}^{m}\left(t_{0}, \cdots, t_{q}\right)=\left(t_{0}, \cdots, t_{m}, 0, t_{m+1}, \cdots, t_{q}\right)
$$

A singular q-simplex of a topological space $X$ is a continuous map $\sigma: \Delta^{q} \rightarrow X$.

Let $G$ be an abelian group. We denote by $S_{q}(X ; \mathbb{Z})$ the free abelian group generated by the singular $q$-simplices $\left\{\sigma: \Delta^{q} \rightarrow X\right\}$ and we set $S_{q}(X ; G)=S_{q}(X ; \mathbb{Z}) \otimes G$. Now, we define the 
differential $\partial_{q}: S_{q}(X ; G) \rightarrow S_{q-1}(X ; G)$ to be the homomorphism defined by its action on singular simplices $\sigma$ by

$$
\partial_{q}(\sigma)=\sum_{m=0}^{q}(-1)^{m} \sigma \circ f_{q}^{m}
$$

Thus, on a chain $\sum_{i=1}^{l} r_{i} \sigma_{i} \in S_{q}(X ; G)$, the differential $\partial$ is given by

$$
\partial\left(\sum_{i=1}^{l} r_{i} \sigma_{i}\right)=\sum_{i=1}^{l} r_{i}\left(\sum_{m=0}^{q}(-1)^{m} \sigma_{i} \circ f_{q}^{m}\right) .
$$

From this definition it follows that $\partial^{2}=0$. By $S_{*}(X ; G)$ we denote the chain complex $\left(S_{q}(X ; G), \partial\right)$, and it is called the singular chain complex with coefficients in $G$. Given a continuous map $f: X \rightarrow Y$ one obtains an induced chain map of complexes $f_{*}: S_{*}(X ; G) \rightarrow S_{*}(Y ; G)$ defined on a singular simplex $\sigma$ by $f_{*}(\sigma)=f \circ \sigma$.

Definition 1.2.6. The homology $H_{*}(X ; G)$ of the singular chain complex $S_{*}(X ; G)$ is defined by

$$
H_{q}(X ; G)=\frac{\operatorname{ker}\left(\partial: S_{q}(X ; G) \rightarrow S_{q-1}(X ; G)\right)}{\operatorname{Im}\left(\partial: S_{q+1}(X ; G) \rightarrow S_{q}(X ; G)\right)} .
$$

The group $H_{*}(X, G)$ is called the singular homology of $X$ with coefficients in $G$. When $G=\mathbb{Z}$, we omit the reference to $G$.

Definition 1.2.7. Let $X$ be a topological space and $A \subset X$ be a subspace of $X$. The relative singular chain complex of the pair $(X, A)$ with coefficients in $G$ is defined by

$$
S_{q}(X, A ; G)=\frac{S_{q}(X ; G)}{S_{q}(A ; G)} .
$$

Remark 1.2.2. $S_{q}(X, A ; G)$ is a free $G$-module. A basis element of $S_{q}(X, A ; G)$ is represented by a singular $q$-simplex in $X$ whose image is not contained in $A$. Moreover, one obtains a commutative diagram with exact rows

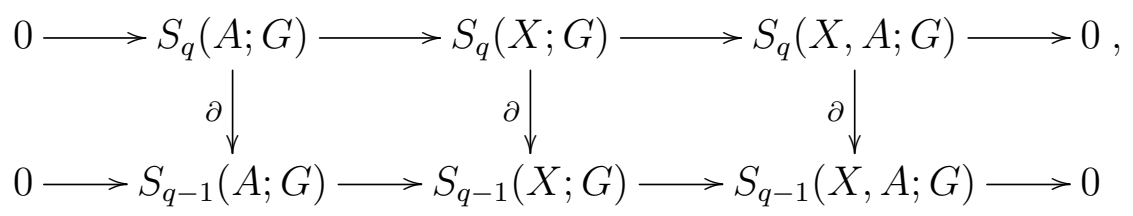

where $\partial: S_{q}(X, A ; G) \rightarrow S_{q-1}(X, A ; G)$ is the map induced by $\partial: S_{q}(X ; G) \rightarrow S_{q-1}(X ; G)$.

The complex $\left(S_{q}(X, A ; G), \partial\right)$ is called the singular chain complex for the pair $(X, A)$ with coefficients in $G$. Its homology is defined by

$$
H_{q}(X, A ; G)=\frac{\operatorname{ker}\left(\partial: S_{q}(X, A ; G) \rightarrow S_{q-1}(X, A ; G)\right)}{\operatorname{Im}\left(\partial: S_{q+1}(X, A ; G) \rightarrow S_{q}(X, A ; G)\right)} .
$$


Lemma 1.2.3 ([1], page 137). If $X$ is a $C W$-complex, then

1. $H_{q}\left(X_{n}, X_{n-1} ; G\right)=0$ for $q \neq n$, and it is free abelian for $q=n$, with a basis in one-to-one correspondence with the $n$-cells of $X$.

2. $H_{q}\left(X_{n} ; G\right)=0$ for $q>n$. In particular, if $X$ is finite-dimensional then $H_{q}(X ; G)=0$ for $q>\operatorname{dim} X$.

3. The inclusion $i: X_{n} \rightarrow X$ induces an isomorphism $i_{*}: H_{q}\left(X_{n} ; G\right) \rightarrow H_{q}(X ; G)$ if $q<n$.

Remark 1.2.3. (see [1]) From the long exact sequences for the pairs $\left(X_{q+1}, X_{q}\right),\left(X_{q}, X_{q-1}\right)$ and $\left(X_{q-1}, X_{q-2}\right)$ one obtains the following sequence

$$
\cdots \rightarrow H_{q+1}\left(X_{q+1}, X_{q} ; G\right) \stackrel{d_{q+1}}{\rightarrow} H_{q}\left(X_{q}, X_{q-1} ; G\right) \stackrel{d_{q}}{\rightarrow} H_{q-1}\left(X_{q-1}, X_{q-2} ; G\right) \stackrel{d_{q-1}}{\rightarrow} \cdots .
$$

Here $d_{q}: H_{q}\left(X_{q}, X_{q-1} ; G\right) \rightarrow H_{q-1}\left(X_{q-1}, X_{q-2} ; G\right)$ is given by the composition

$$
H_{q}\left(X_{q}, X_{q-1} ; G\right) \stackrel{\partial_{q}}{\rightarrow} H_{q-1}\left(X_{q-1}, X_{q-2} ; G\right) \stackrel{i}{\rightarrow} H_{q-1}\left(X_{q-1}, X_{q-2} ; G\right) .
$$

where $\partial_{q}$ is the connection homomorphism in the sequence in singular homology for the pair $\left(X_{q}, X_{q-1}\right)$ and $i$ is induced by the inclusion $\left(X_{q-1}, \phi\right) \rightarrow\left(X_{q-1}, X_{q-2}\right)$

We set $C_{q}(X)=H_{q}\left(X_{q}, X_{q-1} ; G\right)$. Thus, the long exact sequence (1.9) is written as follows

$$
\cdots \rightarrow C_{q+1}(X ; G) \stackrel{\partial_{q+1}}{\rightarrow} C_{q}(X ; G) \stackrel{\partial_{q}}{\rightarrow} C_{q-1}(X ; G) \stackrel{\partial_{q-1}}{\rightarrow} \cdots,
$$

The pair $\left(C_{*}(X ; G), \partial\right)$ is a chain complex. This complex is called the cellular chain complex of $X$, and its homology groups are called the cellular homology groups of $X$. By $H_{q}^{C W}(X ; G)$ we denote the $q$-th cellular homology group of $X$.

Remark 1.2.4. Since $H_{q}\left(X_{q}, X_{q-1} ; G\right)$ is free with a basis in one-to-one correspondence with the $q$ cells of $X$, each of its elements can be thought as a linear combination of $q$-cells of $X$. Thus, the connection map $\partial_{q}: C_{q}(X ; G) \rightarrow C_{q-1}(X ; G)$ is given by the equation

$$
\partial_{q}\left(\sigma_{i}^{q}\right)=\sum_{j} d_{i j} e_{j}^{q-1}
$$

where the sum is over all $(q-1)$-cells $e_{j}^{q-1}$ of $X$ and $d_{i j}$ is the degree of the map $\mathbb{S}_{i}^{q-1} \rightarrow X_{q-1} \rightarrow \mathbb{S}_{j}^{q-1}$, that is the composition of the attaching map of $e_{i}^{q}$ with the quotient map collapsing $X_{q-1} \backslash e_{j}^{q-1}$ to a point.

Theorem 1.2.4 ([1], page 139). $H_{q}^{C W}(X ; G) \cong H_{q}(X ; G)$. 
The cellular cohomology groups of a $C W$-complex $X$ are the algebraic duals of the cellular homology groups of $X$. The cellular cohomology chain complex of $X$ and its cohomology group are given by the following definition.

Definition 1.2.8. Let $X$ be a $C W$-complex and $\left(C_{*}(X ; G), \partial\right)$ be the cellular cochain complex of $X$. Define the cellular cohomology groups with coefficients in the abelian group $G$ by the formula

$$
H^{q}(X ; G)=\frac{\operatorname{ker}\left(\delta: \operatorname{Hom}\left(C_{q}(X ; G), G\right) \rightarrow \operatorname{Hom}\left(C_{q+1}(X ; G), G\right)\right)}{\operatorname{im}\left(\delta: \operatorname{Hom}\left(C_{q-1}(X ; G), G\right) \rightarrow \operatorname{Hom}\left(C_{q}(X ; G), G\right)\right)},
$$

where $\delta$ is defined by $\delta(f)(\sigma)=f(\partial \sigma)$.

Remark 1.2.5. Let $C^{q}(X ; G)=\operatorname{Hom}\left(C_{q}(X ; G), G\right)$. From the definition of $\delta$ follows that $\delta^{2}=0$, and so $\left(C^{*}(X ; G), \delta\right)$ is a complex. Therefore, $H^{q}(X ; G)$ is well defined. This complex is called the cellular cochain complex of $X$.

\subsection{Fiber Bundles}

In this section we will consider fiber bundles and smooth maps. For details we refer the reader to [24], [6], [7], [26].

Definition 1.3.1. Let $E, B, F$ be manifolds. A locally trivial fiber bundle over a manifold $M$ with fiber $F$ is a map $\pi: E \rightarrow M$ with the property that for each point $x \in M$ there exists an open neighborhood $U$ of $x$ in $M$ and a diffeomorphism $\varphi: \pi^{-1}(U) \rightarrow U \times F$ such that the following diagram commutes

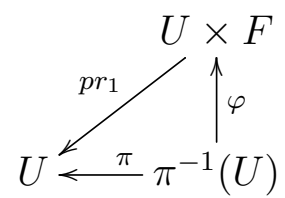

Remark 1.3.1. Terminology and definitions

1. The space $E$ is called the total space, $M$ the base space, $F$ the typical fiber, $\pi$ the projection, and for each $x \in M$, the set $E_{x}=\pi^{-1}(x)$ the fiber over $x$.

2. The map $p r_{1}: M \times F \rightarrow M$ is a fiber bundle with typical fiber $F$ called the trivial bundle.

3. The map $\varphi: \pi^{-1}(U) \rightarrow U \times F$ is called a local trivialization. From the definition 1.3.1 it follows that $\varphi$ has the form $\varphi(p)=(\pi(p), \psi(p))$, for some map $\psi: \pi^{-1}(U) \rightarrow F$. If $\varphi_{\alpha}: \pi^{-1}\left(U_{\alpha}\right) \rightarrow$ $U_{\alpha} \times F$, and $\varphi_{\beta}: \pi^{-1}\left(U_{\beta}\right) \rightarrow U_{\beta} \times F$ are trivializations such that $U_{\alpha} \cap U_{\beta} \neq \phi$, then the composition $\varphi_{\alpha} \circ \varphi_{\beta}^{-1}: U_{\alpha} \cap U_{\beta} \times F \rightarrow U_{\alpha} \cap U_{\beta} \times F$ is given by

$$
\left(\varphi_{\alpha} \circ \varphi_{\beta}^{-1}\right)(x, y)=\left(x, g_{\alpha \beta}(x) y\right),
$$


where $g_{\alpha \beta}: U_{\alpha} \cap U_{\beta} \rightarrow \operatorname{Diff}(F)$ is a smooth map called the transition function. In addition, $\left\{U_{\alpha}\right\}$ is a trivializing open cover of $M$ and $g_{\alpha \beta}$ take values in a subgroup $G \subset \operatorname{Diff}(F)$, then the collection $\left\{g_{\alpha \beta}: U_{\alpha} \cap U_{\beta} \rightarrow G\right\}$ of transition functions satisfies the following relations:

$$
g_{\alpha \beta}(x) g_{\beta \gamma}(x) g_{\gamma \alpha}(x)=e, \text { for all } x \in U_{\alpha} \cap U_{\beta} \cap U_{\gamma} \neq \phi .
$$

The relations (1.14) are called the cocycle conditions.

4. A section of a fiber bundle $\pi: E \rightarrow M$ is a smooth map $s: M \rightarrow E$ such that $\pi \circ s=i d_{M}$. Note that for each $x \in M, s(x) \in \pi^{-1}(x)$.

Definition 1.3.2. A morphism between two fiber bundles $\pi: E \rightarrow M$ and $\pi^{\prime}: E^{\prime} \rightarrow M^{\prime}$ is a pair $(\tilde{f}, f)$, where $\tilde{f}: E \rightarrow E^{\prime}$ and $f: M \rightarrow M^{\prime}$ such that the following diagram commutes

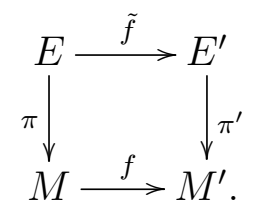

Remark 1.3.2. Other definitions.

1. From the definition it follows that any morphism of fiber bundles maps fibers into fibers, and the map $f: M \rightarrow M^{\prime}$ is determined by $\tilde{f}$.

2. A morphism $(\tilde{f}, f)^{\prime}$ of fiber bundles is called an isomorphism if there exists another morphism $(\tilde{g}, g)$ such that $\tilde{f} \circ \tilde{g}=i d_{E^{\prime}}, \tilde{g} \circ \tilde{f}=i d_{E}$.

3. A bundle $\pi: E \rightarrow M$ with typical fiber $F$ is called trivial if it is isomorphic to the trivial bundle $p r_{1}: M \times F \rightarrow M$.

4. A fiber bundle $p: \tilde{M} \rightarrow M$ with a discrete fiber $\Gamma$ is called a covering. An covering $p: M \times \Gamma \rightarrow$ $M$ is called a trivial covering.

Example 1.3.1. Vector bundles

Definition 1.3.3. A fiber bundle $\pi: E \rightarrow M$ with typical fiber $V$, where $V$ is a finite dimensional vector space, is called a vector bundle if for each $x \in M$ the fiber $\pi^{-1}(x)$ is a vector space, and an atlas exists such that for all trivializations $\varphi: \pi^{-1}(U) \rightarrow U \times V$, the restriction $\varphi: E_{x} \rightarrow\{x\} \times V, x \in U$ is an isomorphism of vector spaces.

Remark 1.3.3. Terminology and examples

1. The dimension of the vector space $V$ is called the rank of the vector bundle. 
2. Let $M$ be a $n$-dimensional manifold and let $T M=\sqcup_{x \in M} T_{x} M$. The map $\pi: T M \rightarrow M$ defined by $\pi(x, v)=x$, where $v \in T_{x} M$, is a vector bundle of rank $n$ called the tangent bundle of $M$. The sections of the tangent bundle are called vectorfields on $M$. When the manifold is a Riemannian manifold, one can consider the subbundle of the tangent bundle $T M$ consisting of all unit tangent vectors. This bundle is called the unit tangent bundle, and we denote it by $\mathbb{S}^{1} T M$.

3. Any vector bundle $\pi: E \rightarrow M$ has the zero section.

\subsubsection{Principal Bundles.}

Another important example of locally trivial fiber bundles is the principal bundle which we study in what follows.

Definition 1.3.4. A locally trivial fiber bundle $\pi: P \rightarrow M$ is called a principal G-bundle if the typical fiber is a Lie group $G$ that acts freely on the right on the total space $P$ in such a way that the base space is the orbit space of this action and each local trivialization from the atlas $\varphi: \pi^{-1}(U) \rightarrow U \times G$ is a $G$-equivariant map, where the action of $G$ on $U \times G$ is given by right multiplication on $G ;\left(x, g_{1}\right) \cdot g_{2}=\left(x, g_{1} g_{2}\right)$.

Remark 1.3.4. 1. For each $x \in M$, the fiber $P_{x}$ is diffeomorphic to the structure group $G$ via the $\operatorname{map} \theta: G \rightarrow P_{x}$ given by $\theta(g)=p \cdot g$, where $\pi(p)=x$.

2. We will also use the notation $P(M, G)$ to refer to a principal $G$-bundle $\pi: P \rightarrow M$ with structure group $G$.

Definition 1.3.5. Let $P_{i}\left(M_{i}, G_{i}\right), i=1,2$, be principal bundles. A morphism $(\tilde{f}, f)$ of fiber bundles joint to a homomorphism of Lie groups $\varphi: G_{1} \rightarrow G_{2}$ is called a morphism of principal bundles if $\tilde{f}(p \cdot g)=\tilde{f}(p) \cdot \varphi(g)$.

Definition 1.3.6. Let $\tilde{f}: P_{1} \rightarrow P_{2}$ be a morphism of two principal bundles $P_{1}\left(M, G_{1}\right)$ and $P_{2}\left(M, G_{2}\right)$ with a common base space $M$. We say that the bundle $P_{1}\left(M_{1}, G_{1}\right)$ is a reduction of the structure group $G_{2}$ of $P_{2}\left(M_{2}, G_{2}\right)$ to $G_{1}$ if $f: P_{1} \rightarrow P_{2}$ is an embedding, and $f: M \rightarrow M$ is the identity map. Proposition 1.3.1. Let $\tilde{f}: P_{1} \rightarrow P_{2}$ be a morphism of two principal bundles $P_{1}(M, G)$ and $P_{2}(M, G)$. If $\varphi=i d_{G}$ and $f=i d_{M}$, then $f$ is an isomorphism.

Proof. Let $\tilde{f}: P_{1} \rightarrow P_{2}$ be a morphism of principal $G$-bundles with the same base space $M$. First of all, we prove that $\tilde{f}$ is a bijection on the fibers. Let $x_{1} \in M$ and $p_{1}, p_{2} \in \pi_{1}^{-1}\left(x_{1}\right)$ such that $\tilde{f}\left(p_{1}\right)=\tilde{f}\left(p_{2}\right)$. Since the action of $G$ is transitive on the fibers, there exists $g \in G$ such that 
$p_{2}=p_{1} \cdot g$. So, $\tilde{f}\left(p_{2}\right)=\tilde{f}\left(p_{1}\right) \cdot g=\tilde{f}\left(p_{2}\right) \cdot g$, and since the action of $G$ on $P_{2}$ is free, we obtain that $g=1$. Thus $p_{1}=p_{2}$, and $\tilde{f}$ is injective. To prove the surjectivity let us $p_{2} \in \pi_{2}^{-1}(x)$ for some $x \in M$. Let $p_{1} \in \pi_{1}^{-1}(x)$ be any element. Since $\pi_{2} \circ f=\pi_{1}$, one obtain that $\tilde{f}\left(p_{1}\right) \in \pi_{2}^{-1}(x)$. Since the action is transitive on the fibers, there exists $g \in G$ such that $\tilde{f}\left(p_{1}\right) \cdot g=p_{2}$, so $\tilde{f}\left(p_{1} \cdot g\right)=p_{2}$, and $\tilde{f}$ is surjective. Thus, we obtain that $\tilde{f}$ is bijective on the fibers, and therefore $\tilde{f}$ is bijective.

On the other hand, since $\pi_{2} \circ \tilde{f}=\pi_{1}$ and $\pi_{1}, \pi_{2}$ are submersions, for each point $p \in P_{1}$, the differential $(d \tilde{f})_{p}$ is injective, and since $P_{1}$ and $P_{2}$ have the same dimension, one obtain that $(d \tilde{f})_{p}$ is an isomorphism. Thus, the inverse function theorem implies that $\tilde{f}$ is a local diffeomorphism. Since $\tilde{f}$ is bijective and a local diffeomorphism, the map $\tilde{f}$ is a diffeomorphism, and hence the morphism $(\tilde{f}, \varphi)$ is an isomorphism of principal $G$-bundles.

Remark 1.3.5. Proposition 1.3.1 holds true for any principal $G_{i}$-bundles $P_{i}\left(M_{i}, G_{i}\right)$ whenever $\varphi$ : $G_{1} \rightarrow G_{2}$ is an isomorphism of groups and the induced map $f: M_{1} \rightarrow M_{2}$ is a diffeomorphism.

Proposition 1.3.2. A principal $G$-bundle $P(M, G)$ is trivial if and only if $P$ admits a section.

Proof. Suppose that $P$ is isomorphic to a trivial bundle as a $G$-bundle, and let $f: P \rightarrow M \times G$ be an isomorphism. We define $s: M \rightarrow P$ by

$$
s(x)=f^{-1}(x, e)
$$

The map $s$ is a section of $P$. In fact, since $\pi=p r_{1} \circ f$, where $p r_{1}: M \times G \rightarrow M$ is the projection on $M$, we have that $(\pi \circ s)(x)=\left(\pi \circ f^{-1}\right)(x, e)=\left(p r_{1} \circ f \circ f^{-1}\right)(x, e)=x$. Moreover, by definition, $s$ is smooth. Conversely, if $s: M \rightarrow P$ is a section of $P$, we set $f: M \times G \rightarrow P$ by the equation

$$
f(x, g)=s(x) \cdot g .
$$

As the action of $G$ on $P$ is smooth, and $s$ is also smooth, we have that $f$ is a smooth map. Moreover, it is easy to prove that $f$ is a $G$-equivariant map. So, by proposition 1.3.1, we only need to prove that $f$ is a morphism of principal $G$-bundles, but it follows from the definition of $f$. In fact, if $\pi: P \rightarrow M$ is the projection map, and $p r_{1}: M \times G \rightarrow M$ is the projection over $M$, then

$$
(\pi \circ f)(x, g)=\pi(s(x) \cdot g)=\pi(s(x))=x=p r_{1}(x, g) .
$$

Therefore, $\pi \circ f=p r_{1}$ and $f$ is a morphism of principal $G$-bundles.

Remark 1.3.6. From the proof of proposition 1.3.2 it follows that any local trivialization $\varphi: \pi^{-1}(U) \rightarrow$ $U \times G$ determines a local section $s:\left.U \rightarrow P\right|_{U}$ which is defined by $s(x)=\varphi^{-1}(x, e)$. Moreover, is $s_{\alpha}:\left.U_{\alpha} \rightarrow P\right|_{U_{\alpha}}$ and $s_{\beta}:\left.U_{\beta} \rightarrow P\right|_{U_{\beta}}$ are local trivializations such that $U_{\alpha} \cap U_{\beta} \neq \phi$, then

$$
s_{\beta}(x)=s_{\alpha}(x) \cdot g_{\alpha \beta}(x),
$$

where $g_{\alpha \beta}: U_{\alpha} \cap U_{\beta} \rightarrow G$ is the corresponding transition function. 


\subsubsection{Associated and induced bundles.}

Let $P(M, G)$ be a principal $G$-bundle. Suppose that $G$ acts on the left on a manifold $Y$. Then $G$ acts on the left on the product manifold $P \times Y$ as follows

$$
g \cdot(p, y)=\left(p \cdot g^{-1}, g \cdot y\right)
$$

The quotient space $(P \times Y) / G=: P \times_{G} Y$ has a structure of differentiable manifold and the projection $\pi_{G}: P \times_{G} Y \rightarrow M$ given by $\pi_{G}([(p, y)])=\pi(p)$ is a fiber bundle with fiber $Y$ ([24], page 54). This bundle is called the fiber bundle with fiber $Y$ associated to $P$. In particular, since $G$ acts on the left on $G / H$, where $H$ is a closed Lie subgroup of $G$, one obtains the bundle $P \times_{G} G / H$.

Proposition 1.3.3 ([24], page 57). Let $P(M, G)$ be a principal bundle and $H$ be a closed Lie subgroup of $G$. Then the space $P / H$ is diffeomorphic to the space $P \times_{G} G / H$.

Proposition 1.3.4 ([24], page 52). Let $M$ be a manifold, $\left\{U_{\alpha}\right\}$ an open covering of $M$ and $G$ a Lie group. Then for each family of functions $\left\{g_{\alpha \beta}: U_{\alpha} \cap U_{\beta} \longrightarrow G\right\}$ which satisfies the relations 1.14 there exists a principal bundle $P(M, G)$ whose transition functions are the functions $g_{\alpha \beta}$.

Let $P(M, G)$ be a principal bundle, $\left\{U_{\alpha}\right\}$ an open covering of $M$ such that $\left.P\right|_{U_{\alpha}}$ is trivial with trivializations $\psi_{\alpha}$ and transition functions $g_{\alpha \beta}$. Let $N$ be a manifold and $f: N \longrightarrow M$ be a smooth map. Then $\left\{f^{-1}\left(U_{\alpha}\right)\right\}$ is an open covering of $N$ and the family of functions $\left\{g_{\alpha \beta} \circ f: f^{-1}\left(U_{\alpha} \cap U_{\beta}\right) \longrightarrow\right.$ $G$ \} satisfies the relations 1.14. Then, by proposition 1.3.4 there exists a principal $G$-bundle over $N$ with transition functions $g_{\alpha \beta} \circ f$. This bundle is called the pullback of $P$ with respect to $f$. We denote it by $f^{*} P$. The total space $f^{*} P$ of the pullback of $P(M, G)$ with respect to $f$ can be written as follows

$$
f^{*} P=\{(x, p) \in N \times P: f(x)=\pi(p)\} .
$$

Moreover, if $\pi_{1}$ and $\pi_{2}$ are the restrictions of the projections of $N \times P$ over $N$ and $P$ to $f^{*} P$, then the following diagram

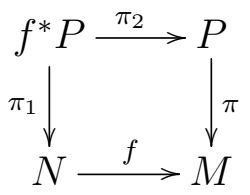

is commutative.

Proposition 1.3.5 ([24], page 60). The projection $\pi_{1}: f^{*} P \rightarrow N$ is a principal G-bundle, and $\pi_{2}: f^{*} P \rightarrow P$ is a morphism of $G$-bundles. Moreover, the bundle $f^{*} P$ is the unique bundle which satisfies the commutative diagram above. 
Remark 1.3.7. Any section $s: M \rightarrow P$ of the principal $G$-bundle $\pi: P \rightarrow M$ determines a section $s^{*}: N \rightarrow f^{*} P$ of the induced bundle $f^{*} P \rightarrow N$. Indeed, this section is given by

$$
s^{*}(x)=(x, s(f(x)))
$$

Theorem 1.3.6 ([24], page 57). Let $P$ be a principal $G$-bundle and let $H$ be a closed Lie subgroup of $G$. Then $G$ has a reduction to a principal $H$-bundle if and only if the associated bundle $P / H$ admits a section.

Proof. Suppose that $P(M, G)$ is reducible to a closed subgroup $H$ and let $Q(M, H)$ be a reduced bundle with injection $f: Q \rightarrow P$. Let $\mu: P \rightarrow P / H$ be the natural projection. First of all, we will prove that the map $\mu \circ f: Q \rightarrow P / H$ is constant on the fibers of $Q$. If $u, v \in \pi_{Q}^{-1}(x)$ for some $x \in M$, we have $v=u \cdot h$ for some $h \in H$. So

$$
(\mu \circ f)(v)=(\mu \circ f)(u \cdot h)=\mu(f(u) \cdot h)=(\mu)(f(u))=(\mu \circ f)(u) .
$$

Since $\pi_{Q}: Q \rightarrow Q / H=M$ is a quotient map, $\mu \circ f$ induces a map $s: M \rightarrow P / H=E$ such that the following diagram is commutative

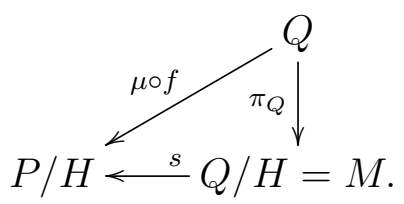

Then $s \circ \pi_{Q}=\mu \circ f$. Now we will show that $s$ is a section of $\pi_{E}: P / H=E \rightarrow M$. Since $\mu \circ f$ and $\pi_{Q}$ are smooth, $s$ is also smooth. Indeed, if $\sigma: U \subset M \rightarrow Q$ is a smooth local section of the bundle $\pi_{Q}: Q \rightarrow Q / H$, then $\left.s\right|_{U}=s \circ i d_{U}=s \circ\left(\pi_{Q} \circ \sigma\right)=\left(s \circ \pi_{Q}\right) \circ \sigma=(\mu \circ f) \circ \sigma$ is smooth. Moreover, since $\pi_{Q}$ is surjective, for any $x \in M$ exists $q \in Q$ such that $\pi_{Q}(q)=x$ we obtain that $s(x)=s\left(\pi_{Q}(q)\right)=\left(s \circ \pi_{Q}\right)(q)=(\mu \circ f)(q)$ and $\left(\pi_{E} \circ s\right)(x)=\pi_{E}((\mu \circ f)(q))=(\pi \circ f)(q)=\pi(f(q))=$ $\pi_{Q}(q)=x$. Conversely, given a section $s: M \rightarrow E$, we consider the bundle $\psi=(\mu: P \rightarrow P / H)$, then the pullback bundle $s^{*} P \rightarrow M$ is a principal $H$-bundle and the map $\tilde{s}: s^{*} P \rightarrow P$ is a morphism of principal bundles. Therefore, $s^{*} P$ is a reduced bundle of $P$.

\subsection{The Bundle of Linear Frames.}

Let $M$ be a $n$-dimensional manifold. Let us consider the set

$$
L(M)=\left\{u: \mathbb{R}^{n} \rightarrow T_{x} M \mid u \text { is a linear isomorphism, } x \in M\right\} .
$$


Recall that for any isomorphism $A: \mathbb{R}^{n} \rightarrow \mathbb{R}^{n}$ the composition $u \circ A: \mathbb{R}^{n} \rightarrow T_{x} M$ is also an isomorphism for all $u \in L(M)$. This defines a right action of $A u t\left(\mathbb{R}^{n}\right)$ on $L(M)$. It is easy to verify that this action is free. Now, since each $u \in L(M)$ is completely determined by its values on a basis of $\mathbb{R}^{n}$, we can identify each element of $L(M)$ with an ordered basis of $T_{x} M$. Therefore, we can write

$$
L(M)=\left\{\left(X_{1}, \cdots, X_{n}\right)_{x} \mid\left(X_{1}, \cdots, X_{n}\right) x \text { is a basis of } T_{x} M \text { at } x, x \in M\right\} .
$$

Now, we have a natural projection $\pi: L(M) \rightarrow M$ given by $\pi\left(\left(X_{1}, \cdots, X_{n}\right)_{x}\right)=x$. By using the canonical isomorphism $A u t\left(\mathbb{R}^{n}\right) \sim G L(n)$ we can see each $A \in \operatorname{Aut}\left(\mathbb{R}^{n}\right)$ as an invertible matrix $\left(a_{i}^{j}\right)$. Therefore, the action above described can be written as follows:

$$
\left(X_{1}, \cdots, X_{n}\right)_{x} \cdot\left(a_{i}^{j}\right)=\left(Y_{1}, \cdots, Y_{n}\right)_{x},
$$

where $Y_{i}=a_{i}^{j} X_{j}$.

Proposition 1.4.1 ([24], page 55). The projection $\pi: L(M) \rightarrow M$ is a principal $G L(n)$-bundle.

The bundle $\pi: L(M) \rightarrow M$ is called the bundle of linear frames on $M$. Some properties of this bundle are the following.

1. The set of reductions of $\pi: L(M) \rightarrow M$ to the orthogonal group $O(n)$ is in one to one correspondence with the set of Riemannian metric on $M$. We denote by $O(M)$ the total space of the reduced bundle, and we call it the bundle of orthogonal frames.

2. If $M$ is an oriented Riemannian $n$-dimensional manifold, the bundle $\pi: L(M) \rightarrow M$ reduces to the special orthogonal group $S O(n)$. In this case the reduced bundle is called the bundle of positively oriented orthonormal frames, and it is denoted by $S O(M)$.

3. Let us consider the standard left action of the group $G L(n)$ on the manifold $\mathbb{R}^{n}$. The bundle with fiber $\mathbb{R}^{n}$ associated to the bundle $L(M)$ is isomorphic to the tangent bundle of $M$. This isomorphism $\tilde{f}: L(M) \times_{G L(n)} T M$ is given by

$$
\tilde{f}\left(\left(X_{1}, \cdots, X_{n}\right)_{x},\left(a_{1}, \cdots, a_{n}\right)\right)=a_{1} X_{1}+\cdots+a_{n} X_{n} .
$$

\subsection{The extension problem.}

This section deals with the extension problem for sections of locally trivial fiber bundles over a finite $C W$-complex $B$, and whose fiber is a manifold $F$ which is simple as a topological space in the sense of observation 1.1.1. 


\subsubsection{The extension problem for maps.}

Let $X$ and $Y$ be topological spaces, $A \subset X$, and $f: A \rightarrow Y$ be a continuous map. The extension problem consists in determine if there exists a continuous map $\tilde{f}: X \rightarrow Y$ such that the following diagram

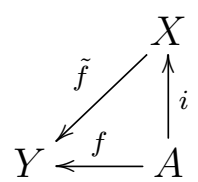

is commutative. The map $\tilde{f}$ is called an extension of $f$.

This problem is closely related to the higher homotopy groups of topological spaces. An important result about this relation is the following

Proposition 1.5.1. Let $X$ be arc connected space, and $h: \mathbb{S}^{n-1} \rightarrow X$ be a continuous map. Then the following conditions are equivalent:

1. $h$ is homotopic to a constant map, i.e, the class $[f]$ is the zero element of the group $\pi_{n-1}(X)$.

2. $h$ extends to a continuous map $k: \mathbb{D}^{n} \rightarrow X$.

Proof. Suppose that $h$ is homotopic to a constant map. Let $c: \mathbb{S}^{n-1} \rightarrow X$ be a constant map homotopic to $h$ and let $H: \mathbb{S}^{n-1} \times I \rightarrow X$ be a homotopy between $h$ and $c$. Consider the map $\pi: \mathbb{S}^{n-1} \times I \rightarrow \mathbb{D}^{n}$ defined by

$$
\pi(x, t)=(1-t) x .
$$

Then $\pi$ is a continuous map, and $\pi$ is a closed map because $\mathbb{S}^{n-1} \times I$ is compact and $\mathbb{D}^{n}$ is Hausdorff. Moreover, $\pi$ is surjective because $\pi(x /|x|, 1-|x|)=x$ for every $x \neq 0$ and $\pi(x, 1)=0$. Therefore, $\pi$ is a quotient map. Note that $\pi^{-1}(0)=\mathbb{S}^{n-1} \times 1$, and $\pi$ is otherwise injective. Since $\left.H\right|_{\mathbb{S}^{n-1} \times 1}$ is constant, then $H$ is constant on the fibers of $\pi$, so $H$ induces a continuous map $k: \mathbb{D}^{n} \rightarrow X$ such that $k \circ \pi=H$ [see [17], page 142]. Hence,

$$
\left.k\right|_{\mathbb{S}^{n-1}}=\left.(k \circ \pi)\right|_{\mathbb{S}^{n-1} \times 0}=\left.H\right|_{\mathbb{S}^{n-1} \times 0}=h .
$$

then $k$ is a continuous extension of $h$.

Conversely, suppose that $h$ extends to a map $k: \mathbb{D}^{n} \rightarrow X$, that is $k \circ i=h$, where $i: \mathbb{S}^{n-1} \rightarrow \mathbb{D}^{n}$ is the inclusion, and let $\pi$ be the map defined by the equation (1.21). We consider the map $H=k \circ \pi$ : $\mathbb{S}^{n-1} \times I \rightarrow X$. Then

$$
\left.H\right|_{\mathbb{S}^{n-1} \times\{1\}}=\left.(k \circ \pi)\right|_{\mathbb{S}^{n-1} \times\{1\}}=k \circ i=h \text { and }\left.H\right|_{\mathbb{S}^{n-1} \times\{0\}}=\left.(k \circ \pi)\right|_{\mathbb{S}^{n-1} \times\{1\}}=\left.k\right|_{\{0\}},
$$

hence $H$ is a homotopy between $h$ and the constant map $x \mapsto k(0)$. 
If the pair $(X, A)$ is a relative $C W$-complex, and $Y$ is a simple path-connected space there exists an algorithm that allows us to solve the extension problem. To solve this problem one can use induction on the dimension of cells of $X$. Since the 0-skeleton is $X^{0}=A \cup\left\{e_{i}^{0}\right\}$, the map $f$ can be extended from $A$ to $X^{0}$ because any function is continuous at isolated points. Now we suppose that $f: A \rightarrow Y$ has been extended to a map $f_{k-1}: X^{k-1} \rightarrow Y$ for some $k \geq 1$, and we wish to extend it to a map $f_{k}: X^{k} \rightarrow Y$. Let $e^{k}:\left(\mathbb{D}^{k}, \mathbb{S}^{k-1}\right) \rightarrow\left(X^{k}, X^{k-1}\right)$ be a $k$-cell of $X$. Note that $f$ is defined over $e^{k}\left(\mathbb{S}^{k-1}\right) \subset X^{k-1}$, so the problem reduces to extend $f$ to $e^{k}\left(\mathbb{D}^{k} \backslash \mathbb{S}^{k-1}\right)$. To visualize this problem consider the following diagram

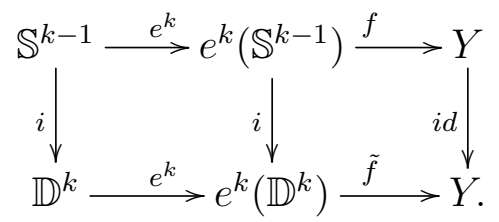

From this diagram we can deduce that such an extension $\tilde{f}: e^{k}\left(\mathbb{D}^{k}\right) \rightarrow Y$ exists if and only if the composition $\left.f \circ e^{k}\right|_{\mathbb{S}^{k-1}}: \mathbb{S}^{k-1} \stackrel{e^{k}}{\rightarrow} e^{k}\left(\mathbb{S}^{k-1}\right) \stackrel{f}{\rightarrow} Y$ extends to a map $\mathbb{D}^{k} \stackrel{e^{k}}{\rightarrow} e^{k}\left(\mathbb{D}^{k}\right) \stackrel{\bar{f}}{\rightarrow} Y$, for each $k$-cell $e^{k}$. Since $Y$ is a simple space, the composition $\left.f \circ e^{k}\right|_{\mathbb{S}^{k-1}}$ defines an element of $\pi_{k-1}(Y)$ (see remark 1.1.1) that we denote by $\alpha\left(e^{k}, f\right)$. Note that $\alpha\left(e^{k}, f\right)$ is zero if and only if the map $f$ can be extended to a map $f_{k}: X^{k} \rightarrow Y$. The elements $\alpha\left(e^{k}, f\right)$ allow us to define a cellular cochain which is an obstruction to the extension of $f$.

Definition 1.5.1. The cellular cochain $\alpha_{f} \in C^{k}\left(X, A, \pi_{k-1}(Y)\right)$ defined by the equation

$$
\alpha_{f}\left(e_{i}^{k}\right)=\alpha\left(e_{i}^{k}, f\right)
$$

is called an obstruction cochain.

The most important properties of the cochain $\alpha_{f}$ defined by the equation (1.23) are given in the following theorem.

Theorem 1.5.2 ([16], page 169). Let $(X, A)$ be a relative $C W$-complex, let $k \geq 1$, and let $Y$ be $a$ connected simple space. Let $f: X^{k-1} \rightarrow Y$ be a continuous map. Then

1. The cochain $\alpha_{f}$ is a cocycle.

2. The cellular cocycle $\alpha_{f} \in C^{k}\left(X, A, \pi_{k-1}(Y)\right)$ vanishes if and only if $f_{k-1}$ extends to a map $f_{k}: X^{k} \rightarrow Y$.

3. The cohomology class $\left[\alpha_{f}\right] \in H^{k}\left(X, A ; \pi_{k-1}(Y)\right)$ vanishes if and only if the restriction $\left.f\right|_{X^{k-2}}$ : $X^{k-2} \rightarrow Y$ extends to a map $f_{k}: X^{k} \rightarrow Y$. 


\subsubsection{The extension problem for sections of fiber bundles.}

In this subsection we apply the mean results of the section 1.5.1 to sections of locally trivial fiber bundles. A most detailled treatment about this topic can be found in [21].

Let $B$ a manifold with a structure of $C W$-complex, $G$ be a connected Lie group, and let $\pi: E \rightarrow B$ be a fiber bundle with structure group $G$, and fiber $F$, where $F$ is a manifold which is simple as a topological space. Let $L \subset B$ be a subcomplex of $B$ such that $(B, L)$ is a relative $C W$-complex, and $s: L \rightarrow E$ be a section of $E$ over $L$. The extension problem consists in determine if $s$ can be extended to a section on $B$. As we did in section 1.5.1, $s$ can be extended to a map $s_{0}: B^{0} \rightarrow E$ by defining $s(x), x \in B^{0} \backslash L$ to be an arbitrary element of the fiber $E_{x}$. This map is a section that extends $s$. Now we suppose that $s$ have been extended to a section $s_{k-1}: B^{k-1} \rightarrow E$, and let $e^{k}:\left(\mathbb{D}^{k}, \mathbb{S}^{k-1}\right) \rightarrow\left(B^{k}, B^{k-1}\right)$ be a $k$-cell of $B$. Since $s_{k-1}: B^{k-1} \rightarrow E$ is defined on $e^{k}\left(\mathbb{S}^{k-1}\right)$, the

problem reduces to extend $s_{k-1}$ from $e^{k}\left(\mathbb{S}^{k-1}\right)$ to $e^{k}\left(\mathbb{D}^{k}\right)$. Let us consider the following commutative diagram

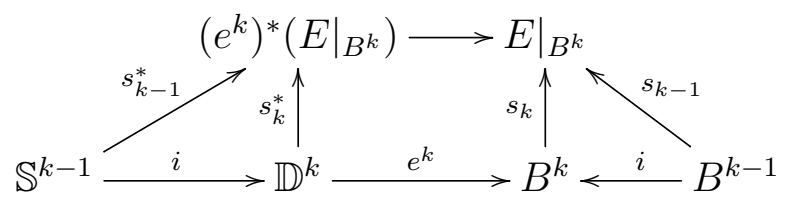

where $s_{k-1}^{*}: \mathbb{S}^{k-1} \rightarrow\left(e^{k}\right)^{*}\left(\left.E\right|_{B^{k}}\right)$ is the section of the pullback bundle $\left(e^{k}\right)^{*} E$ induced by $s_{k-1}$.

Proposition 1.5.3. Let $\pi, s_{k-1}, e^{k}, s_{k-1}^{*}$ be as above. Then $s_{k-1}^{*}$ can be extended to a section $s_{k}^{*}$ : $\mathbb{D}^{k} \rightarrow\left(e^{k}\right)^{*}(E)$ for each cell $e^{k}$ if and only if there exists a section $s_{k}: B^{k} \rightarrow E$ which extends $s_{k-1}: B^{k-1} \rightarrow E$.

Proof. For a section $s_{k}$ of $E$ which extends $s_{k-1}$, we can define the section $s_{k}^{*}: \mathbb{D}^{k} \rightarrow\left(e^{k}\right)^{*}(E)$ (see the equation (1.20)) by

$$
s_{k}^{*}(y)=\left[y, s_{k}\left(e^{k}(y)\right)\right],
$$

and $s_{k}^{*}$ clearly extends $s_{k-1}^{*}$. Conversely, if for all the $k$-cells $e^{k}, s_{k}^{*}: \mathbb{D}^{k} \rightarrow\left(e^{k}\right)^{*}(E)$ is a section of $\left(e^{k}\right)^{*}(E)$ which extends $s_{k}^{*}$, then we define a map $s_{k}: B^{k} \rightarrow E$ by

$$
s_{k}(x)= \begin{cases}s_{k-1}(x) & \text { if } x \in B^{k-1} \\ \left(\pi_{2} \circ s_{k}^{*} \circ\left(e^{k}\right)^{-1}\right)(x) & \text { if } x \in e^{k}\left(\mathbb{D}^{k} \backslash \mathbb{S}^{k-1}\right) .\end{cases}
$$

where $\pi_{2}:\left(e^{k}\right)^{*} E \subset \mathbb{D}^{k} \times E \rightarrow E$ is the restriction of the projection of $\mathbb{D}^{k} \times E$ onto $E$ to $\left(e^{k}\right)^{*} E$. By using the equality $\pi \circ \pi_{2}=e^{k} \circ \pi_{1}$, where $\pi_{1}:\left(e^{k}\right)^{*} E \rightarrow \mathbb{D}^{k}$ is the restriction of the projection of $\mathbb{D}^{k} \times E$ onto $\mathbb{D}^{k}$ to $\left(e^{k}\right)^{*} E$, it is easy to prove that $s_{k}$ is a section of $E$ which extends $s_{k-1}$. 
Let $\varphi:\left(e^{k}\right)^{*}(E) \rightarrow \mathbb{D}^{k} \times F$ be a trivialization of $\left(e^{k}\right)^{*}(E)$, and $p r_{2}: \mathbb{D}^{k} \times F \rightarrow F$ the projection over $F$. The composition map

$$
S: \mathbb{S}^{k-1} \stackrel{s_{k-1}^{*}}{\longrightarrow}\left(e^{k}\right)^{*}(E) \stackrel{\varphi}{\longrightarrow} \mathbb{D}^{k} \times F \stackrel{p r_{2}}{\longrightarrow} F
$$

is a representative of an element in $\pi_{k-1}(F)$ for each $k$-cell $e^{k}$. By proposition 1.5.1, this element is zero in $\pi_{k-1}(F)$ if and only if the section $s_{k-1}^{*}$ extends to a section $s_{k}^{*}$.

Remark 1.5.1. We denote by $\alpha\left(s, e^{k}\right)$ the element of $\pi_{k-1}(F)$ defined by equation (1.26).

Lemma 1.5.4. For each $k$-cell $e^{k}$, the local section $s_{k-1}^{*}$ extends from $\mathbb{S}^{k-1}$ to $\mathbb{D}^{k}$ if and only if $\alpha\left(e^{k}, s\right)=0$.

Proof. Suppose that $s_{k-1}^{*}$ extends to a map $s_{k}^{*}: \mathbb{D}^{k} \rightarrow\left(e^{k}\right)^{*} E$. By proposition 1.5.1, $s_{k-1}^{*}$ is nullhomotopic, and so the composition $S:=\pi_{2} \circ \varphi \circ s_{k-1}^{*}$ (see equation (1.26)) is also nullhomotopic. Hence $\alpha\left(e^{k}, s\right)=0$. Conversely, if $\alpha\left(e^{k}, s\right)=0$, then the map $S: \mathbb{S}^{k-1} \rightarrow F$ given by the equation (1.26) is nullhomotopic, and by proposition 1.5.1 it can be extended to a map $\tilde{S}: \mathbb{D}^{k} \rightarrow F$. Since $S=\pi_{2} \circ \varphi \circ s_{k-1}^{*}$, the map $s_{k-1}^{*}$ can be extended to $\mathbb{D}^{k}$.

Remark 1.5.2. Let $\pi: E \rightarrow B$ be a fiber bundle, $B$ be a path-connected manifold with typical fiber $F$, and $x_{0}, x_{1} \in B$. Any path $\gamma: I \rightarrow B$ from $x_{0}$ to $x_{1}$ determines a homotopy equivalence $\gamma_{*}: E_{x_{0}} \rightarrow E_{x_{1}}$. In particular, we have a homomorphism between $\pi_{1}\left(B, x_{0}\right)$ and the group of homotopy classes of homotopy equivalences of the fiber $E_{x_{0}}$ [see [16], page 117]. Now, since each homotopy equivalence $\gamma_{*}: F \rightarrow F$ induces a bijection $\left[\mathbb{S}^{n}, F\right] \rightarrow\left[\mathbb{S}^{n}, F\right]$, and $\left[\mathbb{S}^{n}, F\right]$ is isomorphic to $\pi_{n}(F)$ because $F$ is simple, we obtain a homomorphism $\pi_{1}(B) \rightarrow \operatorname{Aut}\left(\pi_{n}(F)\right)$. In other words, we have an action of $\pi_{1}(B)$ on $\pi_{n}(F)$ for all $n$ [see [16], page 159]. When this action is trivial, there exists a canonical isomorphism between $\pi_{n}(F)$ and $\pi_{n}\left(F_{x_{0}}\right)$ for all $n$, and all $x_{0} \in B$. Under this condition we have that $\alpha_{s}\left(e_{i}^{k}\right) \in \pi_{k-1}(F)$ for any $k$-cell $e_{i}^{k}$, and so we have a well defined map $\alpha_{s}: C_{k}(B, \mathbb{Z}) \rightarrow \pi_{k-1}(F)$.

In what follows we suppose that this action is trivial. Thus we have the following definition

Definition 1.5.2. We define a cellular $k$-cochain $\alpha_{s} \in C^{k}\left(B^{k}, B^{k-1}, \pi_{k-1}(F)\right)$ by the equation

$$
\alpha_{s}\left(e^{k}\right)=\alpha\left(s, e^{k}\right) .
$$

Proposition 1.5.5. Let $B$ be a manifold with a structure of a finite $C W$-complex and let $\pi: E \rightarrow B$ be a fiber bundle with fiber $F$, where $F$ is a manifold which is simple as a topological space. A local section $s: B^{k-1} \rightarrow E$ of $E$ can be extended to a section over $B^{k}$ if and only if the cellular $k$-cochain $\alpha_{s}$ is zero. 
Proof. It follows from Lemma 1.5.4 and Proposition 1.5.3.

Proposition 1.5.6 ([21], page 167). The cochain $\alpha_{s}$ is a cocycle.

Remark 1.5.3. The cohomology class $\left[\alpha_{s}\right]$ is an obstruction to the extension of $s_{k-1}^{*}$ from $B^{k-1}$ to $B^{k}$. i.e., $\left[\alpha_{s}\right]=0$ if and only if $s_{k-1}^{*}$ can be extended.

Remark 1.5.4. Proposition 1.5.3 and observation 1.5.3 imply that the cohomology class $\left[\alpha_{s}\right]$ is also an obstruction to the extension of $s$ from $B^{k-1}$ to $B^{k}$.

In what follows we will show that $\left[\alpha_{s}\right]$ is independent of the chosen trivializations, and the homotopy class of the section $s$.

Proposition 1.5.7. Let $X$ be a topological space with a left action of a connected Lie group $G$, and let $f_{1}, f_{2}: \mathbb{S}^{k-1} \rightarrow X$ and $g: \mathbb{D}^{k} \rightarrow G$ be continuous maps. If for all $x \in \mathbb{S}^{k-1}$ holds

$$
f_{2}(x)=g(x) \cdot f_{1}(x), \quad x \in \mathbb{S}^{k-1}
$$

then $f_{1}$ and $f_{2}$ are homotopic.

Proof. First of all, we note that $\mathbb{D}^{k}$ is contractible, so the map $g: \mathbb{D}^{k} \rightarrow G$ is nullhomotopic. Let $g_{t}: \mathbb{D}^{k} \rightarrow G$ be a homotopy between the constant map $c: \mathbb{D}^{k} \rightarrow G, x \mapsto e$ and $g$. Since $\left.g\right|_{\mathbb{S}^{k-1}}$ is also homotopic to $c$, the map $H_{t}: \mathbb{S}^{k-1} \rightarrow X$ defined by

$$
H_{t}(x)=g_{t}(x) \cdot f_{1}(x)
$$

is a homotopy between $f_{1}$ and $f_{2}$.

Lemma 1.5.8. The cohomology class $\left[\alpha_{s}\right]$ in $H^{k}\left(B^{k}, B^{k-1}, \pi_{k-1}(F)\right)$ is independent of the chosen trivialization.

Proof. Let $e^{k}:\left(\mathbb{D}^{k}, \mathbb{S}^{k-1}\right) \rightarrow\left(B^{k}, B^{k-1}\right)$ be a $k$-cell on $B$, and $\varphi_{i}:\left(e^{k}\right)^{*}(E) \rightarrow \mathbb{D}^{k} \times F, i=1,2$ be trivializations of the bundle $\mathbb{D}^{k} \rightarrow\left(e^{k}\right)^{*}(E)$. Let $\left[\alpha_{s, 1}\right]$ and $\left[\alpha_{s, 2}\right]$ be the obstruction cohomology classes corresponding to these trivializations. We will show that $\left[\alpha_{s, 1}\right]=\left[\alpha_{s, 2}\right]$. To do it, by the construction of the obstruction cochain is sufficient to show that the maps $S_{i}=\pi_{2} \circ \varphi_{i} \circ s_{k-1}^{*}: \mathbb{S}^{k-1} \rightarrow F, i=1,2$ are homotopic.

Now, if $g: \mathbb{D}^{k} \rightarrow G$ is the transition function of $E$ from $\varphi_{1}$ to $\varphi_{2}$, then $S_{1}$ and $S_{2}$ are related by the equation

$$
S_{2}(x)=g(x) \cdot S_{1}(x), x \in \mathbb{S}^{k-1} .
$$

Since $\mathbb{D}^{k}$ is contractible, by proposition $1.5 .7, S_{1}$ and $S_{2}$ are homotopic.

Proposition 1.5.9. If $s_{1}, s_{2}: B^{k-1} \rightarrow E$ are homotopic local sections, then $\left[\alpha_{s_{1}}\right]=\left[\alpha_{s_{2}}\right]$. 
Proof. Suppose that $s_{1}$ and $s_{2}$ are homotopic. To show that $\left[\alpha_{s_{1}}\right]=\left[\alpha_{s_{2}}\right]$ it is sufficient to show that for each cell $e^{k}$ the sections $s_{1 k}^{*}$ and $s_{2 k}^{*}$ of $\left(e^{k}\right)^{*} E$ are homotopic. Let $H_{t}: B^{k-1} \rightarrow E$ be a homotopy between $s_{1}$ and $s_{2}$, we define a map $\tilde{H}_{t}: \mathbb{S}^{k-1} \rightarrow\left(e^{k}\right)^{*} E$ by

$$
\tilde{H}_{t}(x)=\left(x, H_{t}\left(e^{k}(x)\right)\right) \text {. }
$$

Since $\tilde{H}_{0}(x)=\left(x, H_{0}\left(e^{k}(x)\right)\right)=\left(x, s_{1}\left(e^{k}(x)\right)\right)=s_{1 k}^{*}$ and $\tilde{H}_{1}(x)=\left(x, H_{1}\left(e^{k}(x)\right)\right)=\left(x, s_{2}\left(e^{k}(x)\right)\right)=$ $s_{2 k}^{*}$, the map $\tilde{H}_{t}$ is a homotopy between $s_{1 k}^{*}$ and $s_{2 k}^{*}$. Then for any local trivialization $\varphi$ of $E$ we have that $S_{i}=\pi_{2} \circ \varphi \circ s_{i k}^{*}, i=1,2$ are homotopic for each $k$-cell $e^{k}$, and so $\left[\alpha_{s_{1}}\right]=\left[\alpha_{s_{2}}\right]$.

The principal results of this section are summarized in the following theorem.

Theorem 1.5.10. Let $B$ be a $C W$-complex, $F$ be a manifold which is simple as a topological space and $\pi: E \rightarrow B$ be a fiber bundle with fiber $F$, and let $s: B^{k-1} \rightarrow E$ be a local section of $E$ over the $(k-1)$-skeleton of $B$. Then there exists a cellular cocycle $\alpha_{s} \in C^{k}\left(B^{k}, B^{k-1}, \pi_{k-1}(F)\right)$ which vanishes if and only if $s$ extends to a section $\tilde{s}: B^{k} \rightarrow E$.

Remark 1.5.5. The cohomology class $\left[\alpha_{s}\right] \in H^{k}\left(B^{k}, B^{k-1}, \pi_{k-1}(F)\right)$ vanishes if and only if the restriction of $s$ to $B^{k-2}$ extends to a section $\tilde{s}: B^{k} \rightarrow E$. 


\section{Chapter 2}

\section{Principal $G$-Bundles with Singularities.}

In this chapter we give the definition of principal $G$-bundle with singularities and show that the collection of all principal $G$-bundles with singularities over a base space $M$ and their morphisms form a category. In addition, we prove that the pullback of a principal $G$-bundle with singularities under a map transversal to singularity submanifold is a principal $G$-bundle with singularities. Next, by using the obstruction theory for fiber bundles, we construct a collection of characteristic classes for principal $G$-bundles with singularities.

\subsection{The Category of Principal $G$-bundles with singularities.}

In this section we define principal $G$-bundles with singularities and their morphisms and prove that they form a category.

Definition 2.1.1. Let $\bar{G}$ be a Lie group, let $G \subset \bar{G}$ a Lie subgroup of $G$. Let $M$ be a manifold, and $\Sigma$ be a closed subset of $M$. A principal $\bar{G}$-bundle $\bar{P}(M, \bar{G})$ is called a a principal $G$-bundle with singularities $\Sigma$ if the structure group $\bar{G}$ of $\left.\bar{P}\right|_{M \backslash \Sigma}$ reduces to $G$.

Remark 2.1.1. If $\bar{P}(M, \bar{G})$ is a principal $G$-bundle with singularities and $\Sigma$ is the set of singularities, then the reduction of $\left.\bar{P}\right|_{M \backslash \Sigma}$ to $G$ induces a section $s: M \backslash \Sigma \rightarrow \bar{P} / G$. When $\Sigma$ consists of isolated points, we call the elements of $\Sigma$ isolated singularities of the section $s$ and the principal bundle $\bar{P}$ a principal G-bundle with isolated singularities.

Remark 2.1.2. If $\bar{P}(M, \bar{G})$ is a principal $\{e\}$-bundle with singularities $\Sigma$, then $\left.\bar{P}\right|_{M \backslash \Sigma}$ reduces to a principal bundle $P(M \backslash \Sigma,\{e\})$. From remark 2.1.1 it follows that the bundle $\bar{P} /\{e\}$, which is isomorphic to the principal bundle $\left.\bar{P}\right|_{M \backslash \Sigma}$, admits a section. Hence, $\left.\bar{P}\right|_{M \backslash \Sigma}$ is trivial.

Example 2.1.1. Let $X$ be a vector field on a manifold $M$ of dimension $n, \Sigma \subset M$ be the set of zeros of $X$, and $G$ be the group of affine transformations on $\mathbb{R}^{n-1}$. Then this vector field determines a 
structure of principal $G$-bundle with singularities on the frame bundle $L(M)$ on $M$ : over $M \backslash \Sigma$ the bundle $L(M)$ reduces to the principal $G$-bundle consisting of frames with the first vector equal to the value of $X$ at the corresponding point.

Example 2.1.2. Let $M$ be a Riemannian manifold of dimension $n$. The principal $G L(n)$-bundle of frames $L(M)$ is a principal $O(n)$-bundle with an empty set of singularities.

Example 2.1.3. Let $M$ be a Riemannian two-dimensional manifold, $X$ be a vector field on $M$, and $\Sigma \subset M$ be the set of zeros of $X$. Then $X$ induces a trivialization of the bundle of orthonormal frames $O(M)$ on $M \backslash \Sigma$ to the subgroup $\mathbb{Z}_{2}=\{-I, I\}$ of $O(2)$. Therefore, $X$ determines a structure of principal $\mathbb{Z}_{2}$-bundle with singularities on $O(M)$, where $\Sigma$ is the set of singularities. If, in addition, the manifold $M$ is oriented, then $X$ determines a structure of principal $\{e\}$-bundle with singularities. Example 2.1.4.

Let $M$ be a two dimensional connected Riemannian manifold, and let $\Delta_{1}, \Delta_{2}$ be one dimensional singular distributions on $M$. Suppose that these distributions have the same singular set $\Sigma$, and at each regular point $x \in M \backslash \Sigma$ the lines $\Delta_{1}(x)$ and $\Delta_{2}(x)$ are perpendicular. Let us consider the following set

$$
P=\left\{\left(e_{1}, e_{2}\right)_{x} \in O(M): e_{i} \text { is a generator of } \Delta_{j}(x), x \in M \backslash \Sigma, 1 \leq i, j \leq 2\right\} .
$$

The projection $\pi: P \rightarrow M \backslash \Sigma$ gives a reduction of the bundle of orthonormal frames on $M \backslash \Sigma$ to the subgroup $G=\mathbb{Z}_{2} \ltimes \mathbb{Z}_{4}$. Therefore, $S O(M) \rightarrow M$ is a principal $\mathbb{Z}_{2} \ltimes \mathbb{Z}_{4}$-bundle with singularities. If, in addition, the manifold $M$ is oriented, the bundle $S O(M) \rightarrow M$ is a principal $\mathbb{Z}_{4}$-bundle with singularities.

Example 2.1.5. Let $S$ be an oriented surface in 3-dimensional Euclidean space $\mathbb{R}^{3}$ with isolated umbilical points, and $h$ be the shape operator of $S$. Since $h$ is a symmetric operator with respect to the first fundamental form, for each point $p \in S$ the tangent space $T_{p} S$ has an orthonormal basis consisting of eigenvectors of $h$. These eigenvectors are called principal directions of $S$ at $p$. The points $p$ in $S$ where the eigenvalues of $h$ corresponding to the principal directions coincide are called umbilical points. Let $\Sigma$ be the set of umbilical points on $S$. Let us consider the space

$$
P=\left\{\left(e_{1}, e_{2}\right)_{x} \in S O(S): h\left(e_{i}\right)=k_{i} e_{i}, \text { where } k_{1}>k_{2}, x \in S \backslash \Sigma\right\}
$$

The bundle $\pi_{P}: P \backslash \Sigma \rightarrow S$ given by $\pi_{P}\left(\left(e_{1}, e_{2}\right)_{x}\right)=x$ is a principal $\mathbb{Z}_{2}$-bundle which is a reduction of the bundle of positively oriented orthonormal frames over $S \backslash \Sigma$. Therefore, $S O(S)$ is a principal $\mathbb{Z}_{2}$-bundle with singularities $\Sigma$. 
Definition 2.1.2. Let $\bar{P}_{i}\left(M_{i}, \bar{G}_{i}\right), i=1,2$ be principal $G_{i}$-bundles with singularities, and $\Sigma_{1}$ and $\Sigma_{2}$ be the corresponding sets of singularities. A morphism between $\bar{P}_{1}$ and $\bar{P}_{2}$ is a morphism $\tilde{f}: \bar{P}_{1} \rightarrow \bar{P}_{2}$ (see definition 1.3.5) of principal bundles such that the induced map $f: M_{1} \rightarrow M_{2}$ maps $\Sigma_{1}$ onto $\Sigma_{2}$.

Proposition 2.1.1. The collection of all principal G-bundles with singularities and morphisms between them form a category.

Proof. Let $\bar{P}_{i}\left(M_{i}, \bar{G}_{i}\right), i=1,2,3$ be principal $G_{i}$-bundles with singularities $\Sigma_{i}, i=1,2,3, \tilde{f}: P_{1} \rightarrow P_{2}$ and $\tilde{g}: P_{2} \rightarrow P_{3}$ be morphisms of principal bundles with singularities such that $\tilde{g} \circ \tilde{f}: P_{1} \rightarrow P_{3}$ is defined. Since $\tilde{f}$ and $\tilde{g}$ are morphisms of principal bundles, $\tilde{g} \circ \tilde{f}$ is also a morphism of principal bundles. Moreover, $f\left(\Sigma_{1}\right)=\Sigma_{2}$ and $g\left(\Sigma_{2}\right)=\Sigma_{3}$ imply $(g \circ f)\left(\Sigma_{1}\right)=\Sigma_{3}$. Therefore, $\tilde{g} \circ \tilde{f}$ is a morphism of principal bundles with singularities. It is clear that the class of principal $G$-bundles with singularities and morphisms satisfy all the axioms of category.

Definition 2.1.3. Let $f: X \rightarrow Y$ be a smooth map and $W$ be a submanifold of $Y$. The map $f$ is called transversal to $W$ at the point $p \in X$ if it satisfies one of the following properties:

1. $f(p) \notin W$.

2. $f(p) \in W$ and $T_{f(p)} Y=(d f)_{p}\left(T_{p} X\right)+T_{f(p)} W$.

If $f$ is transversal to $W$ at $p$ for all $p \in X$, we say that $f$ is transversal to $W$.

Proposition 2.1.2 ([2], page 174). Let $f: X \rightarrow Y$ be a smooth map and $W$ be a submanifold of $Y$. If $f$ is transversal to $W$, then $f^{-1}(W)$ is a submanifold of $X$ and $\operatorname{codim}\left(f^{-1}(W)\right)=\operatorname{codim}(W)$.

Proposition 2.1.3. Let $M$ be a compact manifold of dimension n, and let $\bar{P}$ be a principal $G$-bundle over $M$ with singularities $\Sigma$. If $f: N \rightarrow M$ is a map transversal to $\Sigma$, then the pullback bundle $f^{*}(\bar{P})$ is a principal $G$-bundle over $N$ with singularities $f^{-1}(\Sigma)$.

Proof. Let $\bar{P}$ be a principal $G$-bundle with singularities $\Sigma$. We need to prove that the associated fiber bundle $f^{*}(\bar{P}) / G$ admits a section $f^{*} s: N \backslash f^{-1}(\Sigma) \rightarrow f^{*}(\bar{P}) / G$. First of all, note that $f^{-1}(\Sigma)$ is a closed submanifold of $N$ ( see proposition 2.1.2). Now, the following commutative diagram

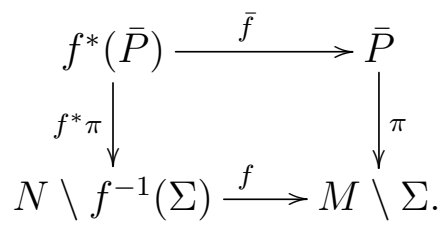

induces a commutative diagram 


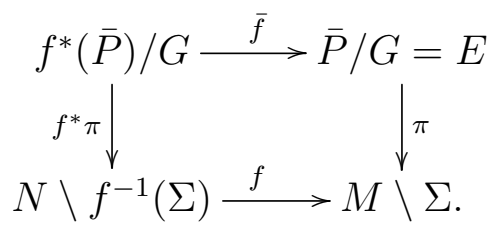

where we denote by $\pi, f^{*} \pi, \bar{f}$ the quotient maps. Now, since $\left.\bar{P}\right|_{M \backslash \Sigma}$ reduces to $G$, the associated fiber bundle $\pi: E \rightarrow M \backslash \Sigma$ admits a section $s: M \backslash \Sigma \rightarrow E$, then the pullback bundle $f^{*} \pi: f^{*}(\bar{P} / G) \rightarrow$ $N \backslash f^{-1}(\Sigma)$ also admits a section $f^{*} s: N \backslash f^{-1}(\Sigma)$. Furthermore, since the bundles $f^{*}(\bar{P} / G)$ and $f^{*}(\bar{P}) / G$ are isomorphic, the bundle $f^{*}(\bar{P}) / G$ admits a section $\tilde{s}: N \backslash f^{-1}(\Sigma)$. More exactly, if $\Psi: f^{*}(\bar{P} / G) \rightarrow f^{*}(\bar{P}) / G$ is such an isomorphism, then we can define a section $\tilde{s}$ by $\tilde{s}=\Psi \circ f^{*} s$. Hence, $f^{*}(\bar{P})$ is a principal $G$-bundle with singularities $f^{-1}(\Sigma)$.

Remark 2.1.3. When the manifolds $M$ and $N$ have the same dimension, and $\bar{P}$ is a principal bundle with isolated singularities, the induced bundle $f^{*} \bar{P}$ is also a principal bundle with isolated singularities.

\subsection{Singularities and Indexes.}

In this section we apply the obstruction theory for sections of fiber bundles to principal $G$-bundles with singularities in order to construct characteristic classes which take their values in homotopy groups of certain quotient of Lie groups. An important fact for the given construction is that the quotient $\bar{G} / G$, where $\bar{G}$ is a Lie group and $G$ is a closed connected Lie subgroup of $\bar{G}$, is simple as topological space ( proposition 1.1.3). In what follows, we assume that $\pi_{1}(M)$ acts trivially on all homotopy groups $\pi_{i}(\bar{G} / G)$, so all of them are canonically identified in the sense of Remark 1.5.2.

\subsubsection{Isolated Singularities and Indexes.}

Let $\bar{G}$ be a connected Lie group, $G$ a closed connected Lie subgroup of $\bar{G}$, let $\pi: P \rightarrow M$ be a principal $G$-bundle with singularities over an $n$-dimensional manifold $M$, and let $T$ be a closed tubular neighborhood of $\Sigma$ in $M$. We choose a $C W$-structure on $T$, and we extend it to a $C W$-structure on $M$ such that the pair $(M, M \backslash \stackrel{\circ}{T})$ is a relative $C W$-complex. From section 1.5.2 it follows that there exists a cohomology class $\left[\alpha_{s}\right] \in H^{*}\left(M, M \backslash \stackrel{\circ}{T}, \pi_{*-1}(\bar{G} / G)\right)$, which is an obstruction to the extension of the section $s: M \backslash \Sigma \rightarrow \bar{P} / G$ corresponding to the reduction of the bundle $\left.\bar{P}\right|_{M \backslash \Sigma}$ ( see Theorem 1.3.6). 
In what follows we assume that $\bar{P}$ is a principal $G$-bundle with isolated singularities and that each $n$-cell $e_{i}^{n}$ of $M$ contains at most one singular point $x_{i} \in \Sigma$. In this case we can assume that the closed tubular neighborhood $T$ of $\Sigma$ is the disjoint union of $n$-cells of $M$ such that each of its cells contains exactly one singular point. In these conditions, the obstruction cohomology class $\left[\alpha_{s}\right]$ is an element of the group $H^{n}\left(M, M \backslash \stackrel{\circ}{T}, \pi_{n-1}(\bar{G} / G)\right)$.

Definition 2.2.1. Let $x_{i} \in \Sigma$ and $e_{i}^{n}$ be the unique $n$-cell of $M$ whose image contains $x_{i}$. The element $\alpha\left(s, e_{i}^{n}\right)$ (see remark 1.5.1) in $\pi_{n-1}(\bar{G} / G)$ is called the index of the section $s$ at the point $x_{i} \in \Sigma$.

Remark 2.2.1. Since the cohomology class $\left[\alpha_{s}\right]$ is independent of the chosen $C W$-complex structure on $M$, the index is well defined.

Example 2.2.1. Let $X$ be a vector field on a two-dimensional oriented compact manifold $M, \Sigma$ the set of zeros of $X$, and let $\bar{P}$ be the principal $\{e\}$-bundle with singularities $\Sigma$ introduced in the example 2.1.3. Since $S O(2) /\{e\}$ is isomorphic to $S O(2), S O(2)$ is homeomorphic to $\mathbb{S}^{1}, \pi_{1}\left(\mathbb{S}^{1}\right)=\mathbb{Z}$, and $\pi_{1}(S O(2) /\{e\})=\mathbb{Z}$. Therefore, the index of each singular point $x \in \Sigma$ is represented by an integer number. This index coincides with the Poincaré's index of zeros of vector fields.

Example 2.2.2. Let $S$ be an oriented surface in the 3-dimensional Euclidean space $\mathbb{R}^{3}, \Sigma$ the set of umbilical points of $S$, and $\bar{P}$ be the principal $\mathbb{Z}_{2}$-bundle with singularities $\Sigma$ given in the example 2.1.5. Then the index of each singular point $x \in \Sigma$ is an element of the fundamental group $\pi_{1}\left(S O(2) / \mathbb{Z}_{2}\right)=$ $\mathbb{Z}$. This index is twice the index of each one dimensional distribution on $S$ generated by principal vectors of $T_{p} S$ on $U \backslash\{x\}$, where $U$ is an open neighborhood of $x$ such that $U \cap \Sigma=\{x\}$.

Proposition 2.2.1. Let $M$ be an oriented compact manifold of dimension n, let $\bar{P}$ be a principal $G$-bundle with singularities $\Sigma$ and let $s: M \backslash \Sigma \rightarrow \bar{P} / G$ be the section induced by the reduction of $\bar{P}_{M \backslash \Sigma}$ to $G$. Then the following assumption are equivalent

1. the sum of the indices of $s$ at the singular points is zero;

2. the obstruction class $\left[\alpha_{s}\right]$ is zero.

Proof. We suppose that $\left[\alpha_{s}\right]=0$. Then by evaluation at the fundamental class $[M]=\sum_{i} e_{i}^{n}$ we obtain

$$
\sum_{i} \alpha_{s}\left(e_{i}^{n}\right)=\alpha_{s}([M])=0 .
$$

Conversely, suppose that the sum of indices of singular points is zero. That is, $\sum_{i} \alpha_{s}\left(e_{i}^{n}\right)=0$. Since the map $\rho_{[M]}: H^{n}\left(M, \pi_{n-1}(\bar{G} / G)\right) \rightarrow H_{0}\left(M, \pi_{n-1}(\bar{G} / G)\right)$ given by $\rho_{[M]}(\varphi)=\varphi([M])$ is an isomorphism, one obtains that $\left[\alpha_{s}\right] \in \operatorname{ker}\left(\rho_{[M]}\right)=\{0\}$. This completes the proof. 


\subsubsection{Singularities of higher dimension and indexes.}

In this section $M$ denotes an $n$-dimensional oriented compact manifold, $\Sigma$ is a $k$-dimensional closed submanifold of $M, \bar{P}$ is a principal $G$-bundle with singularities $\Sigma$, and $s: M \backslash \Sigma \rightarrow \bar{P} / G$ is the section of $\bar{P} / G$ corresponding to the reduction of $\left.\bar{P}\right|_{M \backslash \Sigma}$ to $G$. Let us consider the bundle $\pi: T \rightarrow \Sigma$ with fiber $\mathbb{D}^{n-k}$, where $T \subset M$ is a closed tubular neighborhood. It follows from proposition 1.2.1 that $C W$-complex structures on $\Sigma$ and $\mathbb{D}^{n-k}$ determine a $C W$-complex structure on $T$. In particular, using a $C W$-decomposition of $\Sigma$ and the $C W$-decomposition of $\mathbb{D}^{n-k}$ consisting of a 0-cell $e^{0}$, a $(n-k-1)$-cell $e^{n-k}$ and one $(n-k-1)$-cell $e^{n-k}$, we see the $C W$-decomposition of $T$ contains only cells of the following types:

$$
e^{j}(\Sigma) \times e^{0}, \quad e^{j}(\Sigma) \times e^{n-k-1}, \quad e^{j}(\Sigma) \times e^{n-k}, \quad j=0, \cdots, k,
$$

where by $e^{j}(\Sigma)$ we denote a $j$-cell in $\Sigma$.

Lemma 2.2.2. Let $\bar{P}$ be a principal $G$-bundle with singularities $\Sigma$, and $\pi: T \rightarrow \Sigma$ be a bundle over $\Sigma$, where $T$ is a closed tubular neighborhood of $\Sigma$. Suppose that $T$ has the $C W$-complex structure constructed above. Then first obstruction to the extension of the section $s: M \backslash \operatorname{int}(T) \rightarrow \bar{P} / G$ to $M$ appears in the dimension $n-k$.

Proof. Let us consider the $C W$-decomposition of $T$ given by (2.5). Since the cells $e^{0}$ and $e^{n-k-1}$ belong to the boundary of $\mathbb{D}^{n-k}$, the cells $e^{j}(\Sigma) \times e^{0}$ and $e^{j}(\Sigma) \times e^{n-k-1}$ belong to the boundary of $T$. Moreover, since the section $s: M \backslash \operatorname{int}(T) \rightarrow \bar{P} / G$ is defined on the boundary of $T$, then $\left[\alpha_{s}\right]\left(e^{j}(\Sigma) \times e^{0}\right)=0$, and $\left[\alpha_{s}\right]\left(e^{j}(\Sigma) \times e^{n-k-1}\right)=0$, the possible first obstruction to the extension of $s$ from $M \backslash \operatorname{int}(T)$ to $M$ appears in dimension $n-k$.

Proposition 2.2.3. Let $\Sigma$ be a k-dimensional closed submanifold of $M, \pi: \bar{P} \rightarrow M$ be a principal $G$-bundle with singularities $\Sigma, s: M \backslash \Sigma \rightarrow \bar{P} / G$ the section of $\bar{P} / G$ corresponding to the reduction of $\left.\bar{P}\right|_{M \backslash \Sigma}$ to $G$ and $T$ a closed tubular neighborhood of $\Sigma$. If $s$ is defined over $(M \backslash \operatorname{int}(T)) \cup T^{n-k-1}$, then $(n-k+i)$-cocycle $\alpha_{s}^{(i)}$ which is an obstruction to the extension of $s$ to $(M \backslash T) \cup T^{n-k+i}$ satisfies the following equality

$$
\alpha_{s}^{(i)}\left(\partial e^{i+1}(\Sigma) \times e^{n-k}\right)=0,
$$

for every $(i+1)$-cell $e^{i+1}(\Sigma)$ of $\Sigma$.

Proof. For any $(n-k+i+1)$-cell $e^{i+1}(\Sigma) \times e^{n-k}$ of $T$ we have

$$
\partial\left(e^{i+1}(\Sigma) \times e^{n-k}\right)=\left(\partial e^{i+1}(\Sigma) \times e^{n-k}+(-1)^{i+1} e^{i+1}(\Sigma) \times \partial e^{n-k} .\right.
$$


Since $\alpha_{s}^{(i)}$ is a cocycle, we have that $\alpha_{s}^{(i)}\left(\partial\left(e^{i+1}(\Sigma) \times e^{n-k}\right)\right)=0$. Furthermore, by construction, the cocycle $\alpha_{s}^{(i)}$ also vanishes on $e^{i+1}(\Sigma) \times \partial e^{n-k}$ because this cell lies on the boundary of $T$. Thus, from the equation (2.6) follows the result.

Corollary 2.2.4. Suppose that $\Sigma$ is connected. Then, in the dimension $n-k$, the cocycle $\alpha_{s}$ is constant on the set of all $(n-k)$-cells $e^{0}(\Sigma) \times e^{n-k}$ of $T$, where $e^{0}(\Sigma)$ is any vertex on $\Sigma$.

Proof. Let $e_{i}^{0}(\Sigma)$ and $e_{j}^{0}(\Sigma)$ be two vertices on $\Sigma$. First of all, we suppose that these points are the vertices of an edge $\left[e_{i}^{0}(\Sigma), e_{j}^{0}(\Sigma)\right]$ of $\Sigma$, and we consider the $(n-k+1)$-cell $\left[e_{i}^{0}(\Sigma), e_{j}^{0}(\Sigma)\right] \times e^{n-k}$. Then

$$
\left.\partial\left(\left[e_{i}^{0}(\Sigma), e_{j}^{0}(\Sigma)\right] \times e^{n-k}\right)=-\left[e_{i}^{0}(\Sigma), e_{j}^{0}(\Sigma)\right] \times \partial e^{n-k}+\left(e_{j}^{0}(\Sigma)-e_{i}^{0}\right)(\Sigma)\right) \times e^{n-k} .
$$

Since $\alpha_{s}$ is a cocycle, $\alpha_{s}$ vanishes on $\partial\left(\left[e_{i}^{0}(\Sigma), e_{i+1}^{0}(\Sigma)\right] \times e^{n-k}\right)$. Furthermore, $\alpha_{s}$ also vanishes on $\left[e_{i}^{0}(\Sigma), e_{j}^{0}(\Sigma)\right] \times \partial e^{n-k}$ because this cell belongs to the boundary of $T$, and $s$ is defined over it. Therefore, $\alpha_{s}\left(e_{i}^{0}(\Sigma) \times e^{n-k}\right)=\alpha_{s}\left(e_{j}^{0}(\Sigma) \times e^{n-k}\right)$.

Now suppose that $e_{i}^{0}(\Sigma)$ and $e_{j}^{0}(\Sigma)$ do not belong to the same edge. Since $\Sigma$ is connected, there are vertices $e_{i}^{0}(\Sigma)=e_{1}^{0}(\Sigma), e_{2}^{0}(\Sigma), \cdots, e_{r}^{0}(\Sigma)=e_{j}^{0}(\Sigma)$ and edges $\left[e_{i}^{0}(\Sigma), e_{2}^{0}(\Sigma)\right],\left[e_{2}^{0}(\Sigma), e_{3}^{0}(\Sigma)\right], \cdots,\left[e_{r-1}^{0}(\Sigma), e_{j}^{0}(\Sigma)\right]$ which connect $e_{i}^{0}(\Sigma)$ with $e_{j}^{0}(\Sigma)$. Applying the above result to each edge we complete the proof.

Definition 2.2.2. We call the constant value $\alpha_{s}\left(e^{0}(\Sigma) \times e^{n-k}\right)$ the index of the singularity $\Sigma$, and denote it by $\gamma(\Sigma)$.

Remark 2.2.2. Let us assume that $\Sigma=\Sigma_{1} \cup \cdots \cup \Sigma_{r}$, where each $\Sigma_{i}$ is a closed compact connected submanifold of $M$ of dimension $k$, and $\Sigma_{i}$ are pairwise disjoint, and let $T_{i}$ be a closed tubular neighborhood of $\Sigma_{i}$. Let $T=T_{1} \cup \cdots \cup T_{r}$, and let $\left[\alpha_{s}\right]$ be the obstruction class to the extension of the section $s: M \backslash \operatorname{int}(T) \rightarrow \bar{P} / G$ from $(M \backslash \operatorname{int}(T)) \cup T^{n-k-1}$ to $(M \backslash \operatorname{int}(T)) \cup T^{n-k}$, then $\left[\alpha_{s}\right]$ is constant on the set $\left\{e_{i j_{i}}^{0} \times e^{n-k} \mid e_{i j_{i}}^{0}\right.$ is a vertex of $\left.\Sigma_{i}\right\}$ of $(n-k)$-cells of $T_{i}$. This constant value $\gamma\left(\Sigma_{i}\right)$ is the index of $\Sigma_{i}$.

Example 2.2.3.

Proposition 2.2.5. Let $M$ be an n-dimensional compact connected manifold, and let $\bar{P}=L(M)$ be the principal $G L(n)$-bundle of frames over $M$. If $G=S L(n)$, then the fiber bundle $\bar{P} / G$ is isomorphic to the bundle $\left(\bigwedge^{n}(M)\right)^{o}$, where $\left(\bigwedge^{n}(M)\right)^{o}=\bigwedge^{n}(M) \backslash 0(M)$ and $0: M \rightarrow \bigwedge^{n}(M)$ is the zero section.

Proof. Let $\bar{G}=G L(n), G=S L(n)$ and we note that the map $\bar{G}=G L(n) \rightarrow \mathbb{R}^{*}$ given by $A \mapsto \operatorname{det} A$ induces an isomorphism $\bar{G} / G \cong \mathbb{R}^{*}$. Therefore, we can represent each element of the space $\bar{G} / G$ by a nonzero real number. Now, we define a map $F: \bar{P} / G \cong \bar{P} \times_{\bar{G}} \mathbb{R}^{*} \rightarrow\left(\bigwedge^{n}(M)\right)^{o}$ by the equation

$$
F\left(\left[\left(e_{1}, e_{2}, \cdots, e_{n}\right)_{x}, t\right]\right)=t e^{1} \wedge e^{2} \wedge \cdots \wedge e^{n},
$$


where $\left(e_{1}, e_{2}, \cdots, e_{n}\right)_{x}$ is a frame in $x \in M$, and $\left(e^{1}, e^{2}, \cdots, e^{n}\right)_{x}$ is the dual frame to $\left(e_{1}, e_{2}, \cdots, e_{n}\right)_{x}$. Then $F$ is a bundle isomorphism.

Proposition 2.2.6. Let $M$ be an $n$-dimensional compact connected manifold, let $\bar{P}=L(M)$, and let $w$ be a $n$-form on $M$. Suppose that $\Sigma=\left\{x \in M: w_{x}=0\right\}$ is a $(n-1)$-dimensional compact connected submanifold of $M$, and $P=\left\{\left(e_{1}, e_{2}, \cdots, e_{n}\right) \in \bar{P}_{x}: x \in M, w_{x}\left(e_{1}, e_{2}, \cdots, e_{n}\right)=1\right\}$, then $\bar{P}$ is a principal $S L(n)$-bundle with singularities $\Sigma$.

Proof. We need to prove that the structure group $G L(n)$ of $\left.\bar{P}\right|_{M \backslash \Sigma}$ reduces to $S L(n)$. By proposition 2.2.5, the bundle $\bar{P} / G$ is isomorphic to $\bigwedge^{n}(M) \backslash\{0\}$. Then $w: M \backslash \Sigma \rightarrow \bar{P} / G$ is a section, and the proposition 1.3.6 implies that the structure group of $\bar{P}_{M \backslash \Sigma}$ reduces to $S L(n)$.

Under the conditions of the propositions 2.2.5 and 2.2.6, we consider the problem of extensions of the section $w$ to a non-vanishing section from $M \backslash \Sigma$ to $M$. By obstruction theory, this problem can be solved positively if and only if $w$ can be extended from $(M \backslash T) \cup T^{i-1}$ to $(M \backslash T) \cup T^{i}$ for $i=1,2, \cdots, n$. Since in each step the corresponding cohomology class $\left[\alpha_{w}\right] \in H^{i}\left(M, M \backslash \operatorname{int}(T), \pi_{i-1}(\bar{G} / G)\right)$ is represented by a cochain $\alpha_{w}$ which takes values in $\pi_{i-1}(\bar{G} / G)$, and by obstruction theory, the possible first obstruction can be found in dimension 1 , and also we have that $\pi_{0}(\bar{G} / G)=\mathbb{Z}_{2}$, then $w$ cannot be extended from $(M \backslash T) \cup T^{0}$ to $(M \backslash T) \cup T^{1}$ if and only if $\alpha_{w}$ takes value 1 on at least one 1-cell $e_{i}^{0}(\Sigma) \times e^{1}$, where $e_{i}^{0}(\Sigma)$ is a vertex in $\Sigma$, and $e^{1}$ is the unique 1-cell in the standard $C W$-decomposition of $\mathbb{D}^{1}$. If $\left[\alpha_{w}\right] \in H^{1}(M, M \backslash \operatorname{int}(T)), \pi_{0}(\bar{G} / G)$ is zero, then such an extension exists, and therefore $M$ is orientable. In other dimensions the extension is always possible because $\pi_{i}(\bar{G} / G)=0$ for all $i>0$.

Example 2.2.4. Let $M$ be a two-dimensional compact connected manifold and let $\omega$ be symmetric 2-form. Suppose that $\omega$ is positive definite at all point $x \in M$ where $\omega_{x}$ is not zero. Then $\bar{P}=L(M)$ is a principal $O(2)$-bundle with singularities $\Sigma=\left\{x \in M: \omega_{x}=0\right.$. In this case, the quotient $\bar{G} / G$ is homeomorphic to the vector space of the symmetric matrices, so $\pi_{i}(\bar{G} / G)=0$ for all $i \geq 1$. Therefore, there exists an extension of the section $\omega: M \backslash \Sigma \rightarrow \bar{P} / G$ to a Riemannian metric on $M$. In this case, the index of the singularities is zero.

\subsubsection{Characteristic classes of principal $G$-bundles with singularities.}

Here we give a definition of characteristic classes of principal $G$-bundles with singularities, and we show that the obstruction cohomology class defines a characteristic class on the category of principal bundles with isolated singularities.

Remark 2.2.3. In what follows we denote by $\operatorname{Prin}_{G}^{\Sigma}(M)$ the set of all principal $G$-bundles with singularities $\Sigma$ over $M$. When $\Sigma$ consists only of isolated points we simply write $\operatorname{Prin}_{G}^{0}(M)$. 
Definition 2.2.3. Let $M$ be a manifold. A map $c: \operatorname{Prin}_{G}(M) \rightarrow H^{n}(M)$ is called a characteristic class of principal $G$-bundles with singularities if for any principal $G$-bundle $\bar{P}$ with singularities $\Sigma$ and any smooth map $f: N \rightarrow M$ transversal to $\Sigma$ holds true

$$
f^{*}(c(\bar{P}))=c\left(f^{*}(\bar{P})\right) .
$$

Remark 2.2.4. In the next proposition we denote the section $\tilde{s}$ of $f^{*}(\bar{P}) / G$ defined in the proof of Lemma 2.1 .3 by $f^{*} s$.

Proposition 2.2.7. Let $M$ be a compact manifold of dimension $n, \Sigma$ be a closed submanifold of dimension $k$, and $T$ be a closed tubular neighborhood of $\Sigma$ in $M$. Then, the map $c: \operatorname{Prin}_{G}^{\Sigma}(M) \rightarrow$ $\left.H^{n-k}(M, M \backslash \operatorname{int}(T)) \cup T^{n-k-1}, \pi_{n-k-1}(\bar{G} / G)\right)$ given by $c(P)=\left[\alpha_{s}\right]$ is a characteristic class of principal G-bundles with singularities.

Proof. Let $M$ be an $n$-dimensional manifold, $\Sigma$ be a closed submanifold of $M$ of dimension $k, \bar{P}$ be a principal $G$-bundle on $M$ with singularities $\Sigma, s: M \backslash \Sigma \rightarrow \bar{P} / G$ be the section of $\bar{P} / G$ corresponding to the reduction of $\left.\bar{P}\right|_{M \backslash \Sigma}, f: N \rightarrow M$ be a smooth map transversal to $\Sigma$, and $f^{*} s: N \backslash f^{-1}(\Sigma) \rightarrow$ $f^{*}(\bar{P})$ be the section of $f^{*}(\bar{P})$ induced by $s$ and $f$. We assume that $f$ is a cellular map (Proposition 1.2.2). Recall that $\Sigma^{\prime}:=f^{-1}(\Sigma)$ is the set of singularities of the bundle $f^{*} \bar{P}$ (Proposition 2.1.3). Let $n^{\prime}=\operatorname{dim} N$ and $k^{\prime}=\operatorname{dim} \Sigma^{\prime}$. Let $\alpha_{s}$, and $\alpha_{f^{*} s}$ be the cocycle obstructions to the extension of the section $s$ and $f^{*} s$, respectively. From Proposition 2.1.2 it follows that $n^{\prime}-k^{\prime}=n-k$, so the first obstruction cohomology classes $\left[\alpha_{s}\right]$ and $\left[\alpha_{f^{*}{ }_{s}}\right]$ have degree $n-k$ (Lemma 2.2.2). Let $\Sigma_{1}$ be a connected component of $\Sigma^{\prime}$, and let $T_{1}, T$ be tubular neighborhoods of $\Sigma_{1}, \Sigma$, respectively such that $f\left(T_{1}\right)=T$. Let us consider $C W$-decompositions of $T_{1}$ and $T$ of the type given by the equation (2.5). From the definitions of $\alpha_{s}, \alpha_{f^{*} s}$, and $f^{*} \alpha_{s}$, and Corollary 2.2.4 it follows that in order to show the equality $f^{*}\left[\alpha_{s}\right]=\left[\alpha_{f^{*} s}\right]$ it is sufficient to consider $(n-k)$-cells $e^{\prime}$ in $T_{1}$ such that $e=f\left(e^{\prime}\right)$ is an $(n-k)$-cell in $T$. For such a cell $e$ we have that $\alpha_{f^{*} s_{s}}\left(e^{\prime}\right)$, and $f^{*} \alpha_{s}\left(e^{\prime}\right)$ are represented, respectively, by the maps

$$
\alpha_{f^{*} s}\left(e^{\prime}\right): \mathbb{S}^{n-k-1} \stackrel{\left(e^{\prime}\right)^{*}\left(f^{*} s\right)}{\longrightarrow}\left(e^{\prime}\right)^{*}\left(f^{*}(\bar{P} / G)\right) \stackrel{\varphi}{\longrightarrow} \mathbb{S}^{n-k-1} \times \bar{G} / G \stackrel{p r_{2}}{\longrightarrow} \bar{G} / G,
$$

where $\varphi$ is a trivialization of $\left(e^{\prime}\right)^{*}\left(f^{*}(\bar{P} / G)\right)$, and

$$
\alpha_{s}\left(f\left(e^{\prime}\right)\right): \mathbb{S}^{n-k-1} \stackrel{\left(f \circ e^{\prime}\right)^{*}(s)}{\longrightarrow}\left(f \circ e^{\prime}\right)^{*}(\bar{P} / G) \stackrel{\psi}{\longrightarrow} \mathbb{S}^{n-k-1} \times \bar{G} / G \stackrel{p r_{2}}{\longrightarrow} \bar{G} / G,
$$

where $\psi$ is a trivialization of $\left(f \circ e^{\prime}\right)^{*}(\bar{P} / G)$. Thus, to complete the proof, we need to show that these maps are homotopic. Since the bundles $\left(e^{\prime}\right)^{*}\left(f^{*}(\bar{P} / G)\right) \rightarrow \mathbb{D}^{n-k}$ and $\left(f \circ e^{\prime}\right)^{*}(\bar{P} / G) \rightarrow \mathbb{D}^{n-k}$ are isomorphic, we choose an isomorphism $\tilde{F}:\left(e^{\prime}\right)^{*}\left(f^{*}(\bar{P} / G)\right) \rightarrow\left(f \circ e^{\prime}\right)^{*}(\bar{P} / G)$ such that the induced 
map $F: \mathbb{D}^{n-k} \rightarrow \mathbb{D}^{n-k}$ is the identity map. Thus, we have that $\tilde{F} \circ e^{*} s=\left(e^{\prime}\right)^{*}\left(f^{*} s\right)$, also we can take $\psi=\varphi \circ F$. Therefore, from (2.7) and (2.8) we obtain the following equality:

$$
\alpha_{s}\left(f\left(e^{\prime}\right)\right)=p r_{2} \circ \psi\left(f \circ e^{\prime}\right)^{*} s=p r_{2} \circ \varphi \circ \tilde{F} \circ e^{*} s=p r_{2} \circ \varphi \circ\left(e^{\prime}\right)^{*}\left(f^{*} s\right)=\alpha_{f^{*} s}\left(e^{\prime}\right) .
$$

Thus, we conclude that $f^{*}\left[\alpha_{s}\right]=\left[\alpha_{f^{*} s}\right]$.

Example 2.2.5. Let $M$ be a manifold of dimension $n, \pi: E \rightarrow M$ be an oriented Riemannian vector bundle of rank $m, \Sigma \subset M$ be a closed subset, $\bar{P}=\sqcup_{x \in M} S O\left(\pi^{-1}(x)\right)$, where $S O\left(\pi^{-1}(x)\right)$ is the set of all orthonormal basis of the vector space $\pi^{-1}(x)$, and $s: M \backslash \Sigma \rightarrow E$ be a nonvanishing section of $E$. This section determines a reduction of the bundle $\bar{P} \rightarrow M$ on $M \backslash \Sigma$ to $S O(m-1)$, therefore $\bar{P} \rightarrow M$ is a principal bundle with singularities $\Sigma$. The reduced bundle is given by

$$
P=\left\{\left(e_{1}, \cdots, e_{m}\right)_{y} \in S O(E):\left.e_{1}\right|_{y}=s(x), y \in \pi^{-1}(x), x \in M \backslash \Sigma\right\} .
$$

Since $S O(m) / S O(m-1) \cong \mathbb{S}^{m-1}$, and $\pi_{i}\left(\mathbb{S}^{m-1}\right)=0$ for all $0 \leq i<m-1$, the class $\left[\alpha_{s}\right] \in H^{m}(M, \mathbb{Z})$ is the unique cohomology class which is an obstruction to the existence of a nonvanishing global section of the bundle $E$. This class is the Euler class of the principal bundle with singularities $S O(E)$. 


\section{Chapter 3}

\section{A Generalization of \\ Gauss-Bonnet-Hopf-Poincaré Formula}

In this chapter we give a generalization of the Gauss-Bonnet-Hopf-Poincaré formula Theorem in the context of locally trivial fiber bundles over a two dimensional compact oriented connected manifold. In the first part we define sections with singularities, present a resolution of singularities of such sections, and we also construct an index of singularity point for such sections which generalizes in a natural way the index of zero of a vector field. We prove a generalized version of the Gauss-Bonnet theorem in this context. In the second part, we generalize this result to branched sections of fiber bundles, and we also give a version of the Gauss-Bonnet theorem for these sections.

\subsection{Singularity of section and its index}

\subsubsection{Sections with singularities. Resolution of singularity}

Let $M$ be a 2-dimensional oriented closed manifold, and $\xi=(\pi: E \rightarrow M)$ a locally trivial fiber bundle with typical fiber $F$ and structure group $G$. We will assume that $G$ is a connected Lie group. For each $x \in M$, by $F_{x}$ we will denote the fiber of $\xi$ over $x$, and by $i_{x}: F_{x} \rightarrow E$ the inclusion. Let $\Sigma \subset M$ be a discrete subset. A section with singularities of the bundle $\xi$ is a section $s: M \backslash \Sigma \rightarrow E$. The set $\Sigma$ is called the singularity set of $s$

We assume that there exist a 2-dimensional oriented compact manifold $S$ with boundary $\partial S$ and a map $q: S \rightarrow$ E such that the map q restricted to $\stackrel{\circ}{S}=S \backslash \partial S$ is a diffeomorphism between $S \backslash \partial S$ and $s(M \backslash \Sigma)$. We will call the map $q$ a resolution of the singularities of the section $s$.

Theorem 3.1.1. a) $\pi \circ q: S \backslash \partial S \rightarrow M \backslash \Sigma$ is a diffeomorphism. 


\section{CHAPTER 3. A GENERALIZATION OF GAUSS-BONNET-HOPF-POINCARÉ FORMULA}

b) The map $\pi \circ q: S \rightarrow M$ is surjective.

c) $\pi \circ q(\partial S)=\Sigma$.

d) Let $\partial S=\bigcup_{i=1, k} \partial S_{i}$, where $\partial S_{i}$ are the boundary components. Then for any $x \in \Sigma$, there exist one and only one boundary component $\partial S_{i}$ such that $\pi^{-1}(x)=q\left(\partial S_{i}\right)$.

e) For any $x \in \Sigma$ exists a neighborhood $U$ of $x$ such that

1. $U$ is diffeomorphic to a disk;

2. There exists a closed set $V \subset S$ diffeomorphic to a ring such that the boundary of $V$ consists of two connected components $C$ and $C^{\prime}$, where the connected component $C$ is a closed curve in $S \backslash \partial S$ and the other connected component $C^{\prime}$ is the connected component $\partial S_{i}$ of the boundary $\partial S$ which corresponds to $x \in \Sigma$ by $d$ ), and

$$
\pi \circ q(C)=\partial U, \quad \pi \circ q(V \backslash \partial V)=U \backslash x, \quad \pi \circ q\left(\partial S_{i}\right)=x
$$

Remark 3.1.1. In what follows we will label each point $x \in \Sigma$ such that $\pi^{-1}(x)=q\left(\partial S_{i}\right)$ by the corresponding index $i$.

Proof. a) $s: M \backslash \Sigma \rightarrow s(M \backslash \Sigma)$ is a diffeomorphism and $\left.\pi\right|_{s(M \backslash \Sigma)}: s(M \backslash \Sigma) \rightarrow M \backslash \Sigma$ is the diffeomorphism inverse to $s$. Therefore $\pi \circ q: S \backslash \partial S \rightarrow M \backslash \Sigma$ is a diffeomorphism

b) For each point $x \in M \backslash \Sigma$, the point $y=q^{-1}(s(x)) \in S$ has the property that $\pi \circ q(y)=x$. Let us take a point $x \in \Sigma$ and a sequence of points $\left\{x_{n} \in M \backslash \Sigma\right\}$ converging to $x$. The sequence of points $y_{n}=q^{-1}\left(s\left(x_{n}\right)\right) \in S$ has a convergent subsequence $\left\{y_{n_{k}}\right\}$ because $S$ is compact. If $y=\lim _{n_{k} \rightarrow \infty} y_{n_{k}}$, then

$$
\pi \circ q(y)=\lim _{n_{k} \rightarrow \infty} \pi q\left(y_{n_{k}}\right)=\lim _{n_{k} \rightarrow \infty} x_{n_{k}}=x .
$$

c) As $\pi \circ q$ is surjective and $\pi \circ q(S \backslash \partial S)=M \backslash \Sigma$ because $q(S \backslash \partial S)=s(M \backslash \Sigma)$, we have that $\pi \circ q(\partial S)=\Sigma$.

d) and e) For a point $x \in \Sigma$ let us take a neighborhood $U$ diffeomorphic to an open disk such that $U_{0}=U \backslash\{x\} \subset M \backslash \Sigma$. The closure $\overline{U_{0}}=\Gamma \sqcup U_{0} \sqcup\{x\}$, where $\Gamma$ is the boundary of $U$.

Let $V_{0}=q^{-1} s\left(U_{0}\right)$. Then $\pi \circ q\left(\overline{V_{0}}\right)=\overline{U_{0}}$ because $M$ and $S$ are compact. As $\pi \circ q: S \backslash \partial S \rightarrow M \backslash \Sigma$ is a diffeomorphism, and $\Gamma \subset M \backslash \Sigma$, we have a curve $C \subset\left(\overline{V_{0}} \backslash V_{0}\right) \cap(S \backslash \partial S)$ such that $\pi \circ q(C)=\Gamma$. This means that $C$ is a part of the boundary $\partial V_{0}$ which lies in the interior part of $S$. Now, by b) we know that $\pi^{-1}(x) \subset \sqcup_{i \in I} \partial S_{i}, I \subset\{1, \cdots, n\}$. Finally we have that $\overline{V_{0}}=C \sqcup V_{0} \sqcup\left(\sqcup_{i \in I} \partial S_{i}\right)$, and we know that $V_{0}$ is diffeomorphic to $U_{0}$, and $C \subset S \backslash \partial S$ is diffeomorphic to $\Gamma$, that is $C$ is diffeomorphic to a circle $\mathbb{S}^{1}$. Thus $V_{0}$ is an open set in $S$ diffeomorphic to an open ring, and the boundary of $V_{0}$ consists of a closed curve in the interior part of $S$ and some components of the 
boundary of $S$. However, this means that the boundary of $V_{0}$ contains one and only one component $\partial S_{i}$ of $\partial S$, otherwise the fundamental group of $\pi_{1}\left(V_{0}\right) \neq \mathbb{Z}$. Thus we get the affirmation d) and the decomposition $V=\overline{V_{0}}=C \sqcup V_{0} \sqcup \partial S_{i}$ with the properties given by (2). Therefore, we have proved e).

Remark 3.1.2. From the proof of items d) and e) of Theorem 3.1.1 it follows that, for each boundary component $\partial S_{i}$, we have a closed neighborhood $V_{i}$ of $\partial S_{i}$ and a diffeomorphism of manifolds with boundary $u_{i}: \mathbb{S}^{1} \times[0,1] \rightarrow V_{i}$ such that $u_{i}\left(\mathbb{S}^{1} \times\{1\}\right)=\partial S_{i}, U=\pi \circ q \circ u_{i}\left(\mathbb{S}^{1} \times[0,1]\right)$ is a closed neighborhood of $x_{i} \in \Sigma$ diffeomorphic to a disk, $\pi \circ q \circ u_{i}\left(\mathbb{S}^{1} \times\{0\}\right)$ is the boundary of $U$, and $\pi \circ q \circ u_{i}\left(\mathbb{S}^{1} \times\{1\}\right)=x($ Figure 3.1).

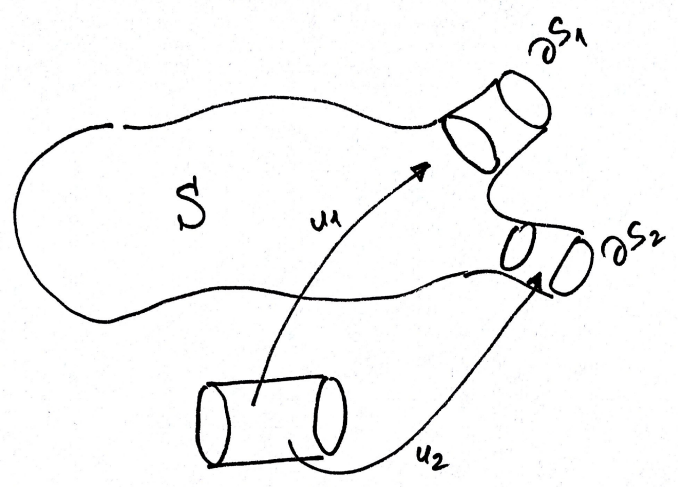

Figure 3.1: The surface $S$

Example 3.1.1. Let $\xi$ be the unit vector bundle of $\mathbb{R}^{2}, \pi: \mathbb{R}^{2} \times \mathbb{S}^{1} \rightarrow \mathbb{R}^{2}$, and consider a section $s: \mathbb{R}^{2} \rightarrow \mathbb{R}^{2} \times \mathbb{S}^{1}, s(\rho, \varphi)=((\rho, \varphi), \cos n \varphi, \sin n \varphi)$ where $(\rho, \varphi)$ is the polar coordinate system on $\mathbb{R}^{2} \backslash(0,0)$. The point $(0,0)$ is the singular point of this section. If we take the coordinate system $(\rho, \varphi, \psi)$ on $\pi^{-1}\left(\mathbb{R}^{2} \backslash(0,0)\right)$, where $\psi$ is the coordinate on $\mathbb{S}^{1}$, then $s(\rho, \varphi)=(\rho, \varphi, n \varphi)$. Now the map $u: \mathbb{S}^{1} \times[0,1] \rightarrow E$ is given with respect to coordinates $(x, y, \psi)$ on $E=\mathbb{R}^{2} \times \mathbb{S}^{1}$ by $u(\alpha, t)=((1-t) \cos \alpha,(1-t) \sin \alpha, n \alpha)$. Therefore $u\left(\mathbb{S}^{1} \times\{0\}\right)$ is a curve consisting of helices, and $u\left(\mathbb{S}^{1} \times\{1\}\right)$ is the fiber $(0,0) \times \mathbb{S}^{1} \subset E$ (fig. 3.2). 


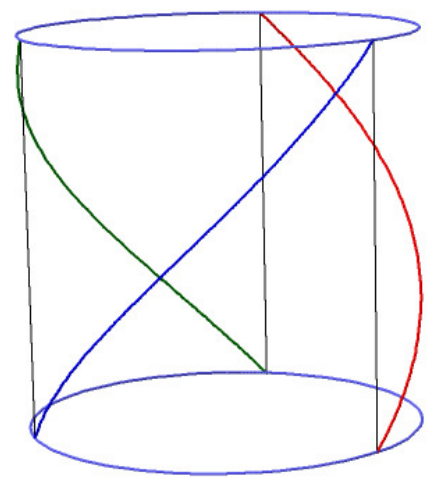

Figure 3.2: Example 3.1.1

\subsubsection{Index of a singular point $x \in \Sigma$.}

First let us observe that the bundle $\xi=(\pi: E \rightarrow M)$ is trivial on the homotopy level. Let $\left(U, \psi: \pi^{-1}(U) \rightarrow U \times F\right)$ be a chart of the atlas of $\xi$. Let

$$
\eta=p r_{2} \circ \psi: \pi^{-1}(U) \rightarrow F,
$$

where $\mathrm{pr}_{2}: U \times F \rightarrow F$ is the canonical projection onto $F$. For each $x \in U$ the map $\eta$ restricted to $F_{x}=\pi^{-1}(x)$ induces a diffeomorphism $\eta_{x}: F_{x} \rightarrow F$. Note that, if we take another chart $\left(U^{\prime}, \psi^{\prime}: \pi^{-1}\left(U^{\prime}\right) \rightarrow U^{\prime} \times F\right)$, and let $\eta^{\prime}: \pi^{-1}\left(U^{\prime}\right) \rightarrow F$ be the corresponding map, then on $\pi^{-1}\left(U \cap U^{\prime}\right)$ we have that

$$
\psi^{\prime} \circ \psi^{-1}:\left(U \cap U^{\prime}\right) \times F \rightarrow\left(U \cap U^{\prime}\right) \times F, \quad(x, y) \mapsto(x, g(x) y),
$$

where $g: U \cap U^{\prime} \rightarrow G$ is the gluing map of the charts. So, for any $x \in U \cap U^{\prime}$, we have $\eta_{x}^{\prime} \circ \eta_{x}^{-1}(y)=$ $g(x) y$, and, as $G$ is connected, $\eta_{x}^{\prime} \circ \eta_{x}^{-1}: F \rightarrow F$ is homotopic to the identity map. This means that for any $x \in M$ we have well defined isomorphisms of the homotopy and (co)homology groups:

$$
\begin{aligned}
& \pi_{*}\left(\eta_{x}\right): \pi_{*}\left(F_{x}\right) \rightarrow \pi_{*}(F), \\
& H_{*}\left(\eta_{x}\right): H_{*}\left(F_{x}\right) \rightarrow H_{*}(F), \quad H^{*}\left(\eta_{x}\right): H^{*}(F) \rightarrow H^{*}\left(F_{x}\right),
\end{aligned}
$$

which do not depend on the chart. In particular, the following diagram is commutative:

$$
\pi_{1}\left(F_{x}\right) \times H^{1}\left(F_{x}\right) \stackrel{\pi_{1}\left(\eta_{x}\right) \times H^{1}\left(\eta_{x}^{-1}\right)}{\longrightarrow} \pi_{1}(F) \times H^{1}(F)
$$




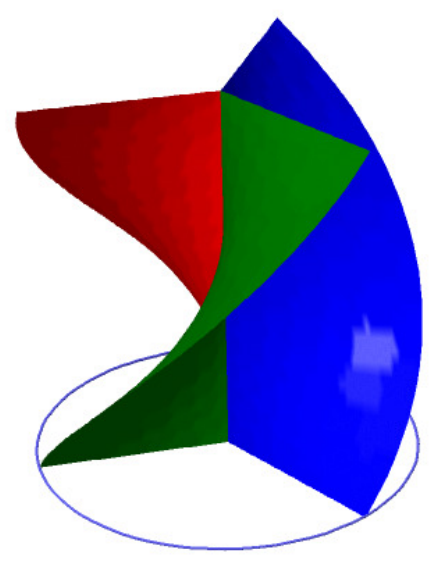

Figure 3.3: Example 3.1.1

where $\langle\cdot, \cdot\rangle$ is the natural pairing through the integration. This means that, for $[\gamma] \in \pi_{1}\left(F_{x}\right)$ and $[\alpha] \in H^{1}(F)$, we have

$$
\left\langle[\gamma], H^{1}\left(\eta_{x}\right)[\alpha]\right\rangle=\int_{\gamma} \eta_{x}^{*} \alpha=\int_{\eta_{x} \gamma} \alpha=\left\langle\pi_{1}\left(\eta_{x}\right)[\gamma],[\alpha]\right\rangle,
$$

and this equality neither depends on the chart, nor on the choice of representatives $\gamma$ or $\alpha$ of their equivalence classes.

Definition 3.1.1. For $x_{i} \in \Sigma$ the map $q \circ u_{i}: \mathbb{S}^{1} \times[0,1] \rightarrow E$ restricted to $\mathbb{S}^{1} \times\{1\}$ gives a map $\gamma_{i}: \mathbb{S}^{1} \rightarrow F_{x_{i}}$, and therefore an element $i n d_{x_{i}}(s) \in \pi_{1}(F)$ via the isomorphism $\pi_{1}\left(\eta_{x}\right)$ (see (3.2)). This element $\operatorname{ind}_{x_{i}}(s)$ will be called the index of the section $s$ at the point $x_{i} \in \Sigma$.

Remark 3.1.3. The index does not depend on the choice of the maps $u_{i}$.

Example 3.1.2. The index of the section $s$ at the point $(0,0)$ in Example 3.1.1 is equal to $n \gamma$, where $\gamma$ is a generator of the group $\pi_{1}\left(\mathbb{S}^{1}\right)$.

Definition 3.1.2. For $a \in H^{1}(F)$ we define the index $\operatorname{ind}_{x_{i}}(s ; a)$ of the section $s$ at the point $x_{i} \in \Sigma$ with respect to $a$ as

$$
\operatorname{ind}_{x_{i}}(s ; a)=\left\langle a, i n d_{x_{i}}(s)\right\rangle=\int_{\gamma} \alpha=\int_{\mathbb{S}^{1}} \gamma^{*} \alpha,
$$

where $\gamma: \mathbb{S}^{1} \rightarrow F$ is the curve representing the element $\operatorname{ind}_{x_{i}}(s) \in \pi_{1}(F)$ and $\alpha \in \Omega^{1}(F)$ is a closed form representing the element $a \in H^{1}(F)$. The index of a section $s$ with respect to $a$ is the number

$$
\operatorname{ind}(s ; a)=\sum_{x_{i} \in \Sigma} \operatorname{ind}_{x_{i}}(s ; a) .
$$


Remark 3.1.4. By (3.5),

$$
\operatorname{ind}_{x_{i}}(s ; a)=\int_{\gamma_{i}} \eta_{x}^{*} \alpha
$$

where $[\alpha]=a$ and $\gamma_{i}$ is defined in Definition 3.1.1.

Example 3.1.3. Let $a=\left[\frac{1}{2 \pi} d \phi\right] \in H^{1}\left(\mathbb{S}^{1} ; \mathbb{R}\right)$, where $d \phi$ is the angular form on $\mathbb{S}^{1}$, then $a$ is a base of $H^{1}\left(\mathbb{S}^{1} ; \mathbb{R}\right)$. Then the index of the section $s$ at the point $(0,0)$ in Example 3.1 .1 with respect to $a$ is equal to $n$.

\subsection{Connection and the Gauss-Bonnet theorem}

Let $\xi=(\pi: E \rightarrow M)$ be a locally trivial fiber bundle over an $n$-dimensional compact manifold $M$, and $F$ be the typical fiber of $\xi$.

\subsubsection{Vertical cohomology class}

Proposition 3.2.1. Let $a \in H^{1}(F)$ and $H$ be a connection in E. There exists a 1 -form $\alpha \in \Omega^{1}(E)$ such that

1. $\left.\alpha\right|_{H}=0$

2. for each $x \in M, d i_{x}^{*} \alpha=0$ and $\left[i_{x}^{*} \alpha\right]=H^{1}\left(\eta_{x}\right) a$.

Proof. Let us take an atlas $\left(U_{i}, \psi_{i}\right)$ of the bundle $\xi$, and fix a 1 -form $\bar{\alpha} \in \Omega^{1}(F)$ such that $[\bar{\alpha}]=a$. Let $\rho_{i}$ be the partition of unity subordinate to the covering $\left\{U_{i}\right\}$, and $\eta_{i}: \pi^{-1}\left(U_{i}\right) \rightarrow F$ (see (3.1)). Then take the collection of 1 -forms $\bar{\alpha}_{i}=\eta_{i}^{*} \bar{\alpha} \in \Omega^{1}\left(\pi^{-1}\left(U_{i}\right)\right)$, and then construct $\widetilde{\alpha}=\sum_{i} \pi^{*} \rho_{i} \bar{\alpha}_{i} \in \Omega^{1}(V)$. Then

$$
i_{x}^{*} \widetilde{\alpha}=\sum_{i} \rho_{i}(x) i_{x}^{*} \bar{\alpha}_{i}=\sum_{i} \rho_{i}(x) i_{x}^{*} \eta_{i}^{*} \bar{\alpha} \in \Omega^{1}\left(F_{x}\right)
$$

Therefore,

$$
d i_{x}^{*} \widetilde{\alpha}=\sum_{i} \rho_{i}(x) i_{x}^{*} \eta_{i}^{*} d \bar{\alpha}=0
$$

and

$$
\left[i_{x}^{*} \widetilde{\alpha}\right]=\sum_{i} \rho_{i}(x)\left[i_{x}^{*} \eta_{i}^{*} \bar{\alpha}\right]=\sum_{i} \rho_{i}(x)\left[\left(\eta_{i}\right)_{x}^{*} \bar{\alpha}\right]=\sum_{i} \rho_{i}(x) H^{1}\left(\eta_{x}\right) a=H^{1}\left(\eta_{x}\right) a,
$$

because $\sum_{i} \rho_{i}(x)=1$.

Since we have the decomposition $T E=H \oplus V$, we can define the desired 1-form $\alpha \in \Omega^{1}(E)$ by $\left.\alpha\right|_{H}=0$ and $\left.\alpha\right|_{V}=\widetilde{\alpha}$. 


\subsubsection{Linear connection in the vertical bundle $V \rightarrow E$}

Let $\Delta$ be a distribution on a manifold $B$. A partial $\Delta$-connection in a vector bundle $\pi: E \rightarrow B$ is given by a covariant derivative:

$$
\nabla: \Delta \times \Gamma(\pi) \rightarrow \Gamma(\pi)
$$

such that

$$
\begin{aligned}
& \nabla_{\lambda X+Y} s=\lambda \nabla_{X} s+\nabla_{Y} s, \quad \lambda \in C^{\infty}(B), X, Y \in \mathfrak{X}(\Delta), s \in \Gamma(\pi) ; \\
& \nabla_{X}\left(s_{1}+s_{2}\right)=\nabla_{X} s_{1}+\nabla_{Y} s_{2} ; X \in \mathfrak{X}(\Delta), s_{1}, s_{2} \in \Gamma(\pi) ; \\
& \nabla_{X}(f s)=X f s+f \nabla_{X} s ; \quad f \in C^{\infty}(B), X \in \mathfrak{X}(\Delta), s \in \Gamma(\pi) .
\end{aligned}
$$

Linear connection in the vector bundle $V \rightarrow E$ induced by the connection $H$.

The connection $H$ induces a partial linear $H$-connection $\nabla^{H}$ in the vector bundle $V \rightarrow E$ :

$$
\nabla_{X}^{H} Y=\omega([X, Y]), \quad X \in \mathfrak{X}^{h}(E), Y \in \mathfrak{X}^{v}(E)
$$

in the following way.

One can easily check that the properties (3.8) hold true. If the connection $H$ satisfies the path lifting property, then the partial $H$-connection has the following geometrical sense. The connection $H$ induces a parallel translation of the fibers of the bundle $E$ along the paths on the base $M$, namely, for $\gamma:[0,1] \rightarrow M$, and for each point $p \in F_{\gamma(0)}$, there exists a unique horizontal lift $\gamma^{h}:[0,1] \rightarrow E$ of $\gamma$ such that $\gamma^{h}(0)=p$, thus we get a diffeomorphism $\Pi_{\gamma}: F_{\gamma(0)} \rightarrow F_{\gamma(1)}, \gamma^{h}(0) \mapsto \gamma^{h}(1)$. Therefore, if a path $\widetilde{\gamma}:[0,1] \rightarrow E$ is tangent to $H$, then $\widetilde{\gamma}$ is a horizontal lift of the path $\gamma=\pi \widetilde{\gamma}:[0,1] \rightarrow M$, so we can define the parallel translation of fibers of the vector bundle $V \rightarrow E$ along the curve $\widetilde{\gamma}$ as follows: $\Pi_{\widetilde{\gamma}}^{H}=\left.d \Pi_{\gamma}\right|_{\gamma^{h}(0)}: V_{\widetilde{\gamma}(0)} \rightarrow V_{\widetilde{\gamma}(1)}$. As $\Pi^{H}$ is linear, from this parallel translation we get a linear partial $H$-connection $\nabla^{H}$ in the bundle $V \rightarrow E$, which is exactly the connection (3.9).

\section{Linear connection in the vector bundle $V \rightarrow E$}

Let us fix a linear partial $V$-connection $\nabla^{V}$ in the bundle $V \rightarrow E$, then the connection $\nabla^{V}$ induces a liner connection in each fiber. We will assume that this connection does not have torsion. Then let us consider the linear connection $\nabla=\nabla^{H}+\nabla^{V}$ in the bundle $V \rightarrow E$ :

$$
\nabla_{X} Y=\nabla_{X^{h}}^{H} Y+\nabla_{X^{v}}^{V} Y
$$

where $X=X^{h}+X^{v}$ corresponds to the decomposition $T E=H \oplus V$, and $Y \in \mathfrak{X}^{v}(E)$. 


\subsubsection{Structure equations of connection}

Proposition 3.2.2. Let $\xi=(\pi: E \rightarrow B)$ be a vector bundle of rank $r$ over a manifold $B$, and $\nabla$ be a linear connection in this bundle. Then we can extend the exterior differential $d: \Omega^{q}(B) \rightarrow \Omega^{q+1}(B)$ to a differential operator $D: \Omega^{q}(B) \otimes \Gamma(\xi) \rightarrow \Omega^{q+1}(B) \otimes \Gamma(\xi)$, where $\Gamma(\xi)$ is the module of sections of $\xi$ so that

$$
D(\omega \otimes s)=d \omega \otimes s+(-1)^{|\omega|} \omega \wedge \nabla s
$$

Proof. Let $\left\{e_{\alpha}\right\}_{\alpha=\overline{1, r}}$ be a local frame field of $\xi$ over $U \subset B$. Then any $\omega \in \Omega^{q}(B) \otimes \Gamma(\xi)$ restricted to $U$ can be written in the form $\omega=\omega^{\alpha} e_{\alpha}$. If we change the frame field to $\left\{e_{\alpha^{\prime}}\right\}_{\alpha^{\prime}=\overline{1, r}}$, then $e_{\alpha^{\prime}}=A_{\alpha^{\prime}}^{\alpha} e_{\alpha}$ and $\omega^{\alpha^{\prime}}=A_{\alpha}^{\alpha^{\prime}} \omega^{\alpha}$, where $A_{\alpha^{\prime}}^{\alpha}$ are functions on $U$. Then,

$$
\begin{aligned}
D\left(\omega^{\alpha^{\prime}} e_{\alpha^{\prime}}\right)=d \omega^{\alpha^{\prime}} e_{\alpha^{\prime}}+(-1)^{q} \omega^{\alpha^{\prime}} \wedge \nabla e_{\alpha^{\prime}}=d\left(A_{\beta}^{\alpha^{\prime}} \omega^{\beta}\right) A_{\alpha^{\prime}}^{\alpha} e_{\alpha}+(-1)^{q} \omega^{\alpha^{\prime}} \wedge \nabla\left(A_{\alpha^{\prime}}^{\alpha} e_{\alpha}\right) \\
=d A_{\beta}^{\alpha^{\prime}} \wedge \omega^{\beta} A_{\alpha^{\prime}}^{\alpha} e_{\alpha}+A_{\beta}^{\alpha^{\prime}} d \omega^{\beta} A_{\alpha^{\prime}}^{\alpha} e_{\alpha}+(-1)^{q} \omega^{\alpha^{\prime}} \wedge d A_{\alpha^{\prime}}^{\alpha} e_{\alpha}+(-1)^{q} \omega^{\alpha^{\prime}} \wedge A_{\alpha^{\prime}}^{\alpha} \nabla e_{\alpha} \\
=\left(A_{\alpha^{\prime}}^{\alpha} d A_{\beta}^{\alpha^{\prime}} \wedge \omega^{\beta}+(-1)^{q} \omega^{\beta} \wedge A_{\beta}^{\alpha^{\prime}} d A_{\alpha^{\prime}}^{\alpha}\right) e_{\alpha}+d \omega^{\alpha} e_{\alpha}+(-1)^{q} \omega^{\alpha} \wedge \nabla e_{\alpha} \\
=\left(A_{\alpha^{\prime}}^{\alpha} d A_{\beta}^{\alpha^{\prime}}+A_{\beta}^{\alpha^{\prime}} d A_{\alpha^{\prime}}^{\alpha}\right) \wedge \omega^{\beta} e_{\alpha}+D\left(\omega^{\alpha} e_{\alpha}\right)=D\left(\omega^{\alpha} e_{\alpha}\right)
\end{aligned}
$$

because $A_{\alpha^{\prime}}^{\alpha} d A_{\beta}^{\alpha^{\prime}}+A_{\beta}^{\alpha^{\prime}} d A_{\alpha^{\prime}}^{\alpha}=d\left(A_{\alpha^{\prime}}^{\alpha} A_{\beta}^{\alpha^{\prime}}\right)=d \delta_{\beta}^{\alpha}=0$.

Remark 3.2.1. $D^{2}(\omega \otimes s)=\omega \wedge R(s)$, where $R$ is the curvature tensor of the linear connection $\nabla$.

Proposition 3.2.3. For $\omega \in \Omega^{1}(B) \otimes \Gamma(E)$, and $X, Y \in \mathfrak{X}(B)$,

$$
D \omega(X, Y)=\nabla_{X}(\omega(Y))-\nabla_{Y}(\omega(X))-\omega([X, Y])
$$

Proof. Let $\left\{e_{\alpha}\right\}$ be a local frame field of $\xi$, and $\omega=\omega^{\alpha} e_{\alpha}$. Then

$$
\nabla_{X}(\omega(Y))=\nabla_{X}\left(\omega^{\alpha}(Y) e_{\alpha}\right)=X\left(\omega^{\alpha}(Y)\right) e_{\alpha}+\omega^{\alpha}(Y) \nabla_{X} e_{\alpha}
$$

therefore

$$
\begin{aligned}
& \nabla_{X}(\omega(Y))-\nabla_{Y}(\omega(X))-\omega([X, Y])= \\
& \begin{aligned}
X\left(\omega^{\alpha}(Y)\right) e_{\alpha}+\omega^{\alpha}(Y) \nabla_{X} e_{\alpha}-Y\left(\omega^{\alpha}(X)\right) e_{\alpha}-\omega^{\alpha}(X) \nabla_{Y} e_{\alpha}-\omega^{\alpha}([X, Y]) e_{\alpha} & = \\
d \omega^{\alpha}(X, Y) e_{\alpha}-\omega^{\alpha} \wedge \nabla e_{\alpha}(X, Y) & =D \omega(X, Y) .
\end{aligned}
\end{aligned}
$$


Now let us assume that we have chosen the linear connection $\nabla$ in the vector bundle $V \rightarrow E$ given by (3.10), and therefore we have the corresponding differential operator $D: \Omega^{q}(B) \otimes \mathfrak{X}^{V}(E) \rightarrow$ $\Omega^{q+1}(B) \otimes \mathfrak{X}^{V}(E)$, where $\mathfrak{X}^{V}(E)$ is the Lie algebra of vertical vector fields.

Proposition 3.2.4 (Structure equations). Let $\omega: T E \rightarrow V$ be the connection form of the connection $H$. Then $\Omega=D \omega \in \Omega^{2}(E) \otimes \mathfrak{X}^{V}(E)$ is a horizontal 2-form on Ewith values on $V E$ given by $\Omega(X, Y)=D \omega\left(X^{h}, Y^{h}\right)$

Proof. It is sufficient to prove that $D \omega(X, Y)=0$ if $Y \in \mathfrak{X}^{v}(E)$. We will apply (3.12). If $X \in \mathfrak{X}^{h}(E)$, then

$$
D \omega(X, Y)=\nabla_{X} \omega(Y)-\nabla_{Y} \omega(X)-\omega([X, Y])=\nabla_{X} Y-\omega([X, Y])=0
$$

by the definition of $\nabla$ (see (3.9) and (3.10)). If $X \in \mathfrak{X}^{v}(E)$, then

$$
D \omega(X, Y)=\nabla_{X} \omega(Y)-\nabla_{Y} \omega(X)-\omega([X, Y])=0
$$

again by the definition of $\nabla$ and because the connection $\nabla^{V}$ is torsion free.

The tensor $\Omega \in \Omega^{2}(E) \otimes \mathfrak{X}^{V}(E)$ is called the curvature form of the connection $H$.

Corollary 3.2.5. The curvature form $\Omega$ vanishes if and only if $H$ is integrable.

Proof. Indeed, by Proposition 3.2.3, we have that, for any horizontal vector fields $X$ and $Y$,

$$
D \omega(X, Y)=-\omega([X, Y]),
$$

therefore $D \omega\left(X^{h}, Y^{h}\right)=0$, for any $X^{h}$ and $Y^{h}$ if and only if the Lie bracket of horizontal vector fields is again a horizontal vector field, this is equivalent to integrability of $H$.

\subsubsection{Generalization of the Gauss-Bonnet-Hopf-Poincaré Formula}

Let $M$ be a 2-dimensional oriented closed manifold, $\Sigma$ be a closed subset of $M, \xi=(\pi: E \rightarrow M)$ a locally trivial fiber bundle with typical fiber $F$ and structure group $G$, and $s: M \backslash \Sigma \rightarrow E$ a secion with singularities

The decomposition $T E=H \oplus V$ induces the bigrading on the space of differential forms on $E$ : $\Omega^{k}(E)=\oplus_{l+m=k} \Omega^{(l, m)}(E)$, and $d: \Omega^{(0, l)}(E) \rightarrow \Omega^{(0, l+1)}(E)$ because the distribution $V$ is integrable.

For $a \in H^{1} F$, let and $\widetilde{\alpha} \in \Omega^{1}(V)$ be a 1-form such that $\eta^{*}\left(\left[\left.\widetilde{\alpha}\right|_{F_{x}}\right]\right)=a$. In Proposition 3.2.1 we have constructed a 1-form $\left.\alpha \in \Omega^{(0,1)} E\right)$ such that $d \alpha$ vanishes on each fiber. Note that the form $\alpha(X)=\widetilde{\alpha}(\omega(X))$ satisfies conditions (1) and (2) of Proposition 3.2.1. 
Let us calculate $d \alpha$. As $X \widetilde{\alpha}(\omega(Y))=\nabla_{X} \widetilde{\alpha}(\omega(Y))+\widetilde{\alpha}\left(\nabla_{X}(\omega(Y))\right.$, we have

$$
\begin{array}{r}
d \alpha(X, Y)=X \alpha(Y)-Y \alpha(X)-\alpha([X, Y])=X \widetilde{\alpha}(\omega(Y))-Y \widetilde{\alpha}(\omega(X))-\widetilde{\alpha}(\omega([X, Y])= \\
\nabla_{X} \widetilde{\alpha}(\omega(Y))+\widetilde{\alpha}\left(\nabla_{X}(\omega(Y))-\nabla_{Y} \widetilde{\alpha}(\omega(X))+\widetilde{\alpha}\left(\nabla_{Y}(\omega(X))-\widetilde{\alpha}(\omega([X, Y])=\right.\right. \\
\nabla_{X} \widetilde{\alpha}(\omega(Y))-\nabla_{Y} \widetilde{\alpha}(\omega(X))+\widetilde{\alpha}(D \omega) .
\end{array}
$$

Here we use Proposition 3.2.3. Let us consider the 2-form

$$
P \in \Omega^{2}(E), \quad P(X, Y)=\nabla_{X} \widetilde{\alpha}(\omega(Y))-\nabla_{Y} \widetilde{\alpha}(\omega(X))
$$

If $X, Y \in \mathfrak{X}^{h}(E)$, then $P(X, Y)=0$. Now if $X, Y \in \mathfrak{X}^{v}(E)$, then $P(X, Y)=d \widetilde{\alpha}(X, Y)=0$. Therefore, the form $P \in \Omega^{(1,1)}(E)$, and for $X \in \mathfrak{X}^{h}(E)$ and $Y \in \mathfrak{X}^{v}(E)$, by (3.15), we get that

$$
P(X, Y)=\nabla_{X} \widetilde{\alpha}(\omega(Y))=X(\widetilde{\alpha}(Y))-\widetilde{\alpha}\left(\nabla_{X} Y\right)=X \alpha(Y)-\alpha([X, Y])=L_{X} \alpha
$$

Now, using Proposition 3.2.3, we arrive at the following proposition.

Proposition 3.2.6. The form $\alpha$ constructed in Proposition 3.2.1 lies in $\Omega^{(0,1)}(E)$ and $d \alpha=\theta_{(1,1)}+$ $\theta_{(2,0)}$, where $\theta_{(1,1)} \in \Omega^{(1,1)}(E)$ and $\theta_{(2,0)} \in \Omega^{(2,0)}(E)$, and

$$
\theta_{(1,1)}(X, Y)=\left(L_{X} \alpha\right)(Y), \quad \theta_{(2,0)}=\widetilde{\alpha}(\Omega)
$$

Note that the forms $\theta_{(1,1)}$ and $\theta_{(2,0)}$ in (3.17) depends only on the connection $H$ and the form $\alpha$. Now we apply the Stokes theorem to the surface $S$ and arrive at a generalization of the Gauss-Bonnet theorem:

Theorem 3.2.7. Let $s$ be a section with singularities of the bundle $\xi=(\pi: E \rightarrow M)$, and $q: S \rightarrow E$ a resolution of the singularities of $s$. For $a \in H^{1}(F)$, let $\alpha \in \Omega^{1}(E)$ be the corresponding vertical form constructed in Proposition 3.2.1. Also let $d \alpha=\theta_{(1,1)}+\theta_{(2,0)}$ as in Proposition 3.2.6. Then

$$
\int_{S} q^{*} \theta_{(1,1)}+q^{*} \alpha(\Omega)=\sum_{i=\overline{1, k}} \operatorname{index}_{x_{i}}(s ; a) .
$$

Proof. By the Stokes theorem,

$$
\int_{S} d q^{*} \alpha=\int_{\partial S} q^{*} \alpha=\sum_{i=1}^{k} \int_{\partial S_{i}} q^{*} \alpha
$$


We have that

$$
\int_{\partial S_{i}} q^{*} \alpha=\int_{\mathbb{S}^{1} \times\{1\}} u_{i}^{*} q^{*} \alpha=\int_{\mathbb{S}^{1}} \gamma_{i}^{*} \alpha=\int_{\gamma_{i}} \alpha=i n d_{x_{i}}(s ; a),
$$

Therefore,

$$
\sum_{i=1}^{k} \int_{\partial S_{i}} q^{*} \alpha=i n d_{x_{i}}(s ; a)
$$

On the other hand, by Proposition 3.2.6,

$$
\int_{S} d q^{*} \alpha=\int_{S} q^{*} d \alpha=\int_{S} q^{*} \theta_{(1,1)}+q^{*} \theta_{(2,0)}
$$

and thus we get the required proposition.

\subsection{Examples}

\subsubsection{Classical Gauss-Bonnet-Hopf-Poincaré Formula}

Let $(M, g)$ be a compact oriented two-dimensional Riemannian manifold, and let

$$
\mathbb{S}^{1} M=\left(\pi: E=T^{1}(M, g) \rightarrow M\right)
$$

be the bundle of unit tangent vectors of $M$, this is a subbundle of the tangent bundle a $M$

Let $\bar{V}(V)$ be the vertical bundle of $T M\left(\mathbb{S}^{1} M\right)$. Let $\iota: \mathbb{S}^{1} M \hookrightarrow T M$ be the inclusion, then $d \iota: T\left(\mathbb{S}^{1} M\right) \rightarrow T(T M)$ induces the vector bundle morphism $\iota_{*}: V \rightarrow \bar{V}$.

Let $H$ be the Levi-Civita connection on the bundle $T M$, and as this connection preserves the metric $g$, the distribution $H$ is tangent to $T^{1} M$, so defines a connection in the bundle $T^{1} M$. Let $\bar{\omega}(\omega)$ be the corresponding connection form on $T M\left(T^{1} M\right)$. Then the following diagram is commutative:

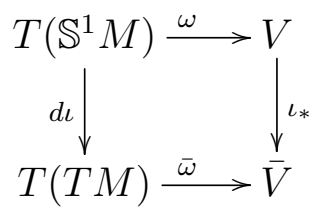

Let us recall that, for each $x \in M$ and $v \in T_{x} M$, we have natural isomorphism $v l: T_{x} M \rightarrow \bar{V}_{v}$ (the vertical lift), $v l(w)=\left.\frac{d}{d t}\right|_{t=0}(v+t w)$ (see [14], 6.11). Let $G$ be the metric on the fibers of $\bar{V}$ defined by $G_{v}\left(w_{1}, w_{2}\right)=g_{\bar{\pi}(v)}\left(v l^{-1}\left(w_{1}\right), v l^{-1}\left(w_{2}\right)\right)$, then using the orientation of $\bar{V}$ lifted from the orientation of $\mathcal{T}_{M}$, we can define the cross-product $\times$ on the fibers of $\bar{V}$. Let us consider the section of $\bar{V}$, for $v \in T M$ we set $E(v)=v l(v) \in \bar{V}_{v}$ (the Liouville vector field). Take the form

$$
\bar{\alpha} \in \Omega^{1}(T M), \quad \bar{\alpha}_{v}(X)=E(v) \times \bar{\omega}_{v}(X), \quad v \in T M, X \in T_{v}(T M),
$$


then $\alpha=\iota^{*} \bar{\alpha} \in \Omega^{1}\left(T^{1} M\right)$ is a form which satisfies the conditions of Statement 3.2.3. Indeed, for each $X \in H, \bar{\omega}(X)=0$, therefore $\alpha(X)=0$. At the same time, for each $x \in M$, we have an isometry $\eta$ of $\mathbb{R}^{2}$ with the standard metric to the fiber $T_{x} M$ with the metric $g_{x}$. This isometry maps $\mathbb{S}^{1} \subset \mathbb{R}^{2}$ to the fiber $T_{x}^{1} M$, and it is easy to see that $\eta^{*} \alpha$ is the standard angle form on $\mathbb{S}^{1}$, and this fact does not depend on the choice of the isometry $\eta$. Now let us find the expression of $\alpha$ is terms of local trivializations.

The bundle $\mathbb{S}^{1} M$ is a locally trivial bundle with structure group $S O(2)$ and typical fiber $\mathbb{S}^{1}$. The bundles $T M$ and $\mathbb{S}^{1} M$ trivialize simultaneously over an open subset $U \subset M$ if and only if there exists an orthonormal frame field $\left\{e_{1}, e_{2}\right\}$ on $U$, in this case the trivialization of $T M$ given by the diffeomorphism:

$$
\bar{f}: U \times \mathbb{R}^{2} \rightarrow \bar{\pi}^{-1}(U), \quad\left(x,\left(v^{1}, v^{2}\right)\right) \mapsto v^{1} e_{1}(x)+v^{2} e_{2}(x)
$$

induces the trivialization $f: U \times \mathbb{S}^{1} \rightarrow \bar{\pi}^{-1}(U)$ of $\mathcal{T}_{M}^{1}$ such that the following diagram

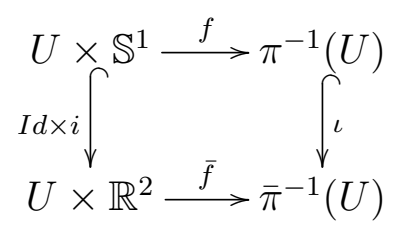

where $i: \mathbb{S}^{1} \hookrightarrow \mathbb{R}^{2}$ is the standard inclusion, is commutative. The trivialization $\bar{f}$ determines the projection $\bar{p} r: \bar{\pi}^{-1}(U) \rightarrow \mathbb{R}^{2}$, and $f$ determines the projection $p r: \pi^{-1}(U) \rightarrow \mathbb{S}^{1}$.

Now on $U$, for an orthonormal frame field $e_{a}, a=1,2$, we have

$$
\nabla e_{1}=G e_{2}, \quad \nabla e_{2}=-G e_{1}
$$

where $G$ is a 1 -form on $U$. Note that $d G=K e^{1} \wedge e^{2}$, where $K$ is the Gauss curvature, and $\left\{e^{a}\right\}$ is the coframe dual to the frame $\left\{e_{a}\right\}$.

In terms of the trivialization $\bar{f}$ the connection form $\omega$ on $\bar{\pi}^{-1}(U)$ is written as follows: $\bar{\omega}=\bar{\omega}^{a} E_{a}$, where $\bar{\omega}^{1}=d v^{1}-v^{2} \bar{\pi}^{*} G, \bar{\omega}^{2}=d v^{2}+v^{1} \pi^{*} G$, and $E_{a}=v l\left(e_{a}\right)$.

From (3.20) it follows that $\bar{\alpha}=-v^{2} \omega^{1}+v^{1} \omega^{2}$, and simple calculation shows that

$$
\left.d \bar{\alpha}=2 d v^{1} \wedge d v^{2}+\left(v^{1} d v^{1}+v^{2} d v^{2}\right) \wedge \bar{\pi}^{*} G+\left(\left(v^{1}\right)^{2}+\left(v^{2}\right)^{2}\right)\right) \bar{\pi}^{*} d G
$$

As $\pi^{-1}(U)=\left\{\bar{f}\left(x,\left(v^{1}, v^{2}\right)\right) \mid\left(v^{1}\right)^{2}+\left(v^{2}\right)^{2}=1\right\}$, we have that $d \alpha=i^{*} d \bar{\alpha}=i^{*} \bar{\pi}^{*} d G=\pi^{*} d G=$ $\pi^{*}\left(K e^{1} \wedge e^{2}\right)$.

Now let us take a vector field $W \in \mathfrak{X}(M)$. Then $W$ defines a section $s=\frac{1}{\|W\|} W: M \backslash \Sigma \rightarrow E$, where $\Sigma$ is the set of zeros of $W$. Assume that the set $\Sigma$ is discrete and therefore finite, so $\Sigma=$ $\left\{x_{1}, \cdots, x_{k}\right\}$. For each $x_{i} \in \Sigma$ we fix an orientation preserving diffeomorphism $c_{i}:(D, 0) \rightarrow\left(U_{i}, x_{i}\right)$, 
where $D \subset \mathbb{R}^{2}$ is a disk centered at the origin 0 . Then the bundle $T M$ is trivial over $U_{i}$, and let $\left\{e_{a}^{i}\right\}, a=1,2$, be the corresponding orthonormal frame field over $U_{i}$, and $f_{i}: U_{i} \times \mathbb{S}^{1} \rightarrow \pi^{-1}\left(U_{i}\right)$ the trivialization (see (3.21) and (3.22)). Now let us consider the map

$$
t_{i}: \mathbb{S}^{1} \times[0,1] \rightarrow U_{i}, \quad\left(\left(y^{1}, y^{2}\right), \rho\right) \mapsto c_{i}\left((1-\rho) y^{1},(1-\rho) y^{2}\right),
$$

and let $\psi_{i}: U_{i} \backslash\left\{x_{i}\right\} \rightarrow \mathbb{S}^{1}, \psi_{i}(x)=\left(y_{i}^{1}(x), y_{i}^{2}(x)\right)$, where $\left(y^{1}\right)^{2}+\left(y^{2}\right)^{2}=1$, be the local representation of the section $s$ with respect to the trivialization $f_{i}$, this means that

$$
s(x)=y_{i}^{1}(x) e_{1}^{i}(x)+y_{i}^{2}(x) e_{2}^{i}(x)=f_{i}\left(x,\left(y_{i}^{1}(x), y_{i}^{2}(x)\right)\right) \quad \forall x \in U_{i} .
$$

Now $t_{i}$ induces a diffeomorphism $\mathbb{S}^{1} \times[0,1) \rightarrow U_{i} \backslash\left\{x_{i}\right\}$ and therefore we get a map $\psi_{i} \circ t_{i}: \mathbb{S}^{1} \times[0,1) \rightarrow$ $\mathbb{S}^{1}$. Now assume that the map $\psi_{i} \circ t_{i}$ can be prolonged to a map $\Psi_{i}: \mathbb{S}^{1} \times[0,1] \rightarrow \mathbb{S}^{1}$. Under this assumption we can construct a resolution of the singularities of the section $s$, this means a compact 2dimensional manifold $S$ with boundary $\partial S$ and a map $q: S \rightarrow E$ such that $q$ restricted to $S^{\prime}=S \backslash \partial S$ is a diffeomorphism between $S^{\prime}$ and $s\left(M^{\prime}\right)$, where $M^{\prime}=M \backslash \Sigma$ (see Section 3.1).

Remark 3.3.1. In geometric terms, our assumption means that the angle $\psi$ of the unit vector field $s$ with the vector field $e_{1}$ (see (3.26)) has limit when we approach to the point $x_{i}$ by some curve, but the limit depends on the curve.

In order to construct $S$, first we take $S^{\prime}=M \backslash \cup_{i=1}^{k} U_{i}$, this is a submanifold of $M$ whose boundary consists of the curves $\Gamma_{i}=\partial U_{i}=c_{i}(\partial D)$. Then take the disjoint union $S^{\prime \prime}$ of $k$ copies of the cylinder $\mathbb{S}^{1} \times[0,1] \cong \partial D \times[0,1]$, and attach $S^{\prime \prime}$ to $S^{\prime}$ using the map $C: \sqcup_{i=1}^{k} \partial D \times 0 \rightarrow \sqcup_{i=1}^{k} \Gamma_{i}$ determined by the diffeomorphisms

$$
\left.c_{i}\right|_{\partial D}: \mathbb{S}^{1} \times\{0\} \cong \partial D \times\{0\} \rightarrow \Gamma_{i} .
$$

Thus we get the manifold $S=S^{\prime \prime} \cup_{C} S^{\prime}$. Now the map $q: S \rightarrow E$ is defined as follows: for each $p \in S^{\prime}$, we set $q(p)=s(p)$, and for each $p \in S^{\prime \prime}=\sqcup_{i=1}^{k} \partial D \times[0,1]$, if $p$ belongs to $i$-th copy of $\mathbb{S}^{1} \times[0,1] \cong \partial D \times[0,1]$, we set $q\left(\left(\left(z^{1}, z^{2}\right), a\right)\right)=f_{i}\left(t_{i}\left(\left(z^{1}, z^{2}\right), a\right), \Psi_{i}\left(\left(z^{1}, z^{2}\right), a\right)\right)$, where $\left(z^{1}, z^{2}\right) \in \mathbb{S}^{1}$, $a \in[0,1]$. From the construction it is clear that $q$ is well defined on $S^{\prime} \cap S^{\prime \prime}=\sqcup_{i=1}^{k} \Gamma_{i}$.

Denote by $\Psi_{i}^{a}: \mathbb{S}^{1} \rightarrow \mathbb{S}^{1}$ the map $\Psi_{i}: \mathbb{S}^{1} \times[0,1] \rightarrow \mathbb{S}^{1}$ restricted to $\mathbb{S}^{1} \times\{a\}$. Then $\Psi_{i}^{0}$ is homotopic to $\Psi_{i}^{1}$, therefore $\operatorname{deg} \Psi_{i}^{0}=\operatorname{deg} \Psi_{i}^{1}$. However, $\Psi_{i}^{0}=\psi_{i} \circ t_{i}$ restricted to $\mathbb{S}^{1} \times\{0\}$ and its degree coincides with the degree of $\psi_{i}$ restricted to $\Gamma_{i}=\partial U_{i}$, therefore the $\operatorname{deg} \Psi_{i}^{0}$ is equal to the classical index $\operatorname{Ind}_{x_{i}}(s)$ of the vector field $s$ at the point $x_{i}$. Therefore,

$$
\operatorname{ind}_{x_{i}}(s, a)=\int_{\gamma_{i}} \alpha=\int_{\mathbb{S}^{1}}\left(\Psi_{i}^{1}\right)^{*} \theta=\operatorname{deg} \Psi_{i}^{1}=\operatorname{deg} \Psi_{i}^{0}=\operatorname{Ind}_{x_{i}}(s) .
$$


This means that the classical index $\operatorname{Ind}_{x_{i}}(s)$ of vector field $s$ at the singular point $x_{i}$ coincides with the index $\operatorname{ind}_{x_{i}}(s, a)$. On the other hand $d \alpha=\pi^{*} d G=\pi^{*} K e^{1} \wedge e^{2}$, where $K$ is the curvature of the metric $g$. Therefore, in Statement 3.2.4 $\theta_{(1,1)}=0$ and $\alpha(\Omega)=\pi^{*}\left(K e^{1} \wedge e^{2}\right)$. Thus, Statement 3.2.4 and (3.28) gives us

$$
\int_{S} q^{*} \pi^{*}\left(K e^{1} \wedge e^{2}\right)=\sum_{i=\overline{1, k}} \operatorname{Ind}_{x_{i}}(s) .
$$

At the same time, by the construction, $\pi \circ q: S \backslash \partial S \rightarrow M \backslash \Sigma$ is a diffeomorphism, $\Sigma$ is a set of measure zero, and $K e^{1} \wedge e^{2}$ is a 2-form defined globally on $M$, therefore

$$
\int_{S} q^{*} \pi^{*}\left(K e^{1} \wedge e^{2}\right)=\int_{M} K e^{1} \wedge e^{2}
$$

and we get the classical result

$$
\int_{M} K e^{1} \wedge e^{2}=\sum_{i=\overline{1, k}} \operatorname{Ind}_{x_{i}}(s) .
$$

\subsubsection{Gauss-Bonnet-Hopf-Poincaré Formula for Projective Bundles}

\section{Projective bundles}

Definition 3.3.1. A projective bundle is a locally trivial bundle $Q \rightarrow M$ with typical fiber $\mathbb{R} P^{n}$ and structure group $P G L(n)$. The number $n$ is called the rank of $Q$.

Example 3.3.1. Let $E \rightarrow M$ be a vector bundle. Then the bundle $P(E)$ of 1-dimensional subspaces of the fibers of $E$ is called the projectivization of $E$ and it is a projective bundle.

Remark 3.3.2. Projective bundles and projective connections were consider by B. N. Shapukov in [5]

\section{Projective bundle as projectivization of a vector bundle}

If we have a projective bundle $Q \rightarrow M$, can we find a vector bundle $E$ such that $Q=P(E)$ ? Note that for $A \in G L(n+1)$, we have $\operatorname{det}(\lambda A)=\lambda^{n+1} \operatorname{det} A$. Now for an even $n$, the group $P G L(n)=G L(n+1) / A \sim \lambda A, \lambda \in \mathbb{R}$, is isomorphic to $S L(n+1)$ because for each equivalence class $[A] \in P G L(n)$ one can find a representative $B=(\operatorname{det} A)^{-1 /(n+1)} A \in[A]$ such that $\operatorname{det} B=1$. This $B$ is unique because for $B, B^{\prime} \in[A] \operatorname{such}$ that $\operatorname{det} B=\operatorname{det} B^{\prime}=1$, we have that $B^{\prime}=\lambda B$ and $\lambda^{n+1}=1$, this implies that $\lambda=1$. Therefore in this case $P G L(n) \cong S L(n+1)$. For an odd $n$, the group $P G L(n)=P G L_{+}(n) \sqcup P G L_{-}(n)$, where

$$
P G L_{+}(n)=\{[A] \mid \operatorname{det} A>0\}, \quad P G L_{-}(n)=\{[A] \mid \operatorname{det} A<0\} .
$$


By the same arguments as for case of even $n$ we get that $P G L_{+}(n) \cong S L(n) / \pm I$. Note that $P G L_{-}(n)$ is diffeomorphic to $P G L_{+}(n)$. If the structure group $P G L(n)$ of a projective bundle $Q$ reduces to $P G L_{+}(n)$, then $Q$ is called orientable, on the contrary $Q$ is called non orientable.

Example 3.3.2. If a vector bundle $E$ of even rank is non orientable, then $P(E)$ is non orientable.

Now let consider the following situation. Let $\widetilde{G}$ be a Lie group, $Z=Z(\widetilde{G})$ be the center of $\widetilde{G}$, and $G=\widetilde{G} / Z$. So we have the exact sequence

$$
0 \longrightarrow Z \stackrel{i}{\longrightarrow} \widetilde{G} \stackrel{p}{\longrightarrow} G \longrightarrow 0 \text {. }
$$

Let $\pi: P \rightarrow M$ be a principal $G$-bundle, $\mathcal{U}=\left\{U_{i}\right\}$ be a good covering of a manifold $M$, and $g_{i j}: U_{i j} \rightarrow M$ be the transition functions of $P$. As the covering $\mathcal{U}$ is good, we can lift $g_{i j}$ up to $\widetilde{g}_{i j}: U_{i j} \rightarrow \widetilde{G}$ of $g_{i j}$, this means that the following diagram is commutative

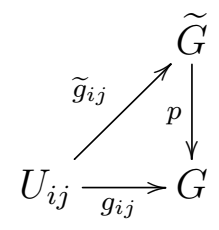

Consider the Cech cochain $z=\left\{z_{i j k}: U_{i j k} \rightarrow \widetilde{G}\right\}$ over the covering $\mathcal{U}$, where

$$
z_{i j k}=\widetilde{g}_{i j} \widetilde{g}_{j k} \widetilde{g}_{k i} \text {. }
$$

As $p\left(z_{i j k}\right)=g_{i j} g_{j k} g_{k i}=1$, the functions $z_{i j k}$ take values in $Z$, therefore in fact $z \in \check{C}^{2}(\mathcal{U} ; Z)$.

Proposition 3.3.1. 1. The Čech cochain $z$ is a normalized cocycle.

2. The $\check{C}$ ech cohomology class $[z] \in \check{H}^{2}(M ; Z)$ is trivial if and only if there exists a $\widetilde{G}$-principal bundle $\widetilde{P}$ such that $P \cong \widetilde{P} / Z$.

Proof. Let us prove that $\left\{z_{i j k}\right\}$ is a Čhech cocycle on $\mathcal{U}$. First of all note that we have

$$
\widetilde{g}_{i j} \widetilde{g}_{j k}=z_{i j k} \widetilde{g}_{i k}=\widetilde{g}_{i k} z_{i j k} .
$$

Then

$$
\left(\widetilde{g}_{i j} \widetilde{g}_{j k}\right) \widetilde{g}_{k i}=z_{i j k} \widetilde{g}_{i k} \widetilde{g}_{k i}=z_{i j k} \text { and } \widetilde{g}_{i j}\left(\widetilde{g}_{j k} \widetilde{g}_{k i}\right)=\widetilde{g}_{i j} \widetilde{g}_{j i} z_{j k i}=z_{j k i},
$$

therefore $z_{i j k}=z_{k i j}=z_{j k i}$. At the same time,

$$
z_{i j k}^{-1} \widetilde{g}_{i k}^{-1}=\left(z_{i j k} \widetilde{g}_{i k}\right)^{-1}=\left(\widetilde{g}_{i j} \widetilde{g}_{j k}\right)^{-1}=\widetilde{g}_{k j} \widetilde{g}_{j i}=z_{k j i} \widetilde{g}_{k i},
$$


hence follows that $z_{i j k}^{-1}=z_{k j i}=z_{j i k}$. Moreover $z_{i j k}=1$ if at least two indices coincide. Thus $z=\left\{z_{i j k}\right\}$ is a normalized cochain. Now we have

$$
\left(\widetilde{g}_{i j} \widetilde{g}_{j k}\right)\left(\widetilde{g}_{k j} \widetilde{g}_{j l}\right)=z_{i j k} \widetilde{g}_{i k} z_{k j l} \widetilde{g}_{k l}=z_{i j k} z_{k j l} z_{i k l} g_{i l}
$$

and

$$
\left(\widetilde{g}_{i j} \widetilde{g}_{j k}\right)\left(\widetilde{g}_{k j} \widetilde{g}_{j l}\right)=\left(\widetilde { g } _ { i j } \left(\widetilde{g}_{j k}\left(\widetilde{g}_{k j}\right) \widetilde{g}_{j l}=\widetilde{g}_{i j} \widetilde{g}_{j l}=z_{i j l} \widetilde{g}_{i l},\right.\right.
$$

thus $z_{i j k} z_{k j l} z_{i k l}=z_{i j l}$, this implies

$$
\delta z=z_{j k l} z_{i k l}^{-1} z_{i j l} z_{i j k}^{-1}=0 .
$$

If $z=\delta u$, where $u \in \check{C}^{1}(\mathcal{U} ; Z)$, then

$$
z_{i j k}=u_{i j} u_{j k} u_{k i}
$$

Therefore we can construct the $\widetilde{G}$-principal bundle $\widetilde{P} \rightarrow M$ with the transition functions $\widetilde{g}_{i j}^{\prime}=\widetilde{g}_{i j} u_{j i}$ because

$$
\widetilde{g}_{i j}^{\prime} \widetilde{g}_{j k}^{\prime} \widetilde{g}_{k i}^{\prime}=\widetilde{g}_{i j} u_{j i} \widetilde{g}_{j k} u_{k j} \widetilde{g}_{k i} u_{i k}=\widetilde{g}_{i j} \widetilde{g}_{j k} \widetilde{g}_{k i} u_{j i} u_{k j} u_{i k}=z_{i j k} u_{j i} u_{k j} u_{i k}=1 .
$$

Now we have that $p\left(\widetilde{g}_{i j}^{\prime}\right)=g_{i j}$ and from the following lemma it follows that there exists a principal bundle morphism $\pi: \widetilde{P} \rightarrow P$ written with respect to the trivializations as $(x, \widetilde{g}) \rightarrow(x, p(\widetilde{g}))$.

Lemma 3.3.2. Let $G_{a}, a=1,2$ be Lie groups. Let $\pi_{a}: P_{a} \rightarrow M, a=1,2$ be a $G_{a}$-principal bundle and $f: G_{1} \rightarrow G_{2}$ be a Lie group homomorphism. Assume that $\mathcal{U}=\left\{U_{i}\right\}$ is a trivializing covering for $P_{1}$ and $P_{2}$ at the same time. If the transition functions $g_{i j}^{a}$ of $P_{a}$ with respect to the covering $\mathcal{U}$ are related by $f\left(g_{i j}^{1}\right)=g_{i j}^{2}$, then there exists a principal fiber bundle homomorphism $F: P_{1} \rightarrow P_{2}$ corresponding to $f$, i. e. $F\left(p_{1} g_{1}\right)=F\left(p_{1}\right) f\left(g_{1}\right)$.

Proof. We define $F_{i}: \pi_{1}^{-1}\left(U_{i}\right) \rightarrow \pi_{2}^{-1}\left(U_{i}\right)$ by the commutative diagram

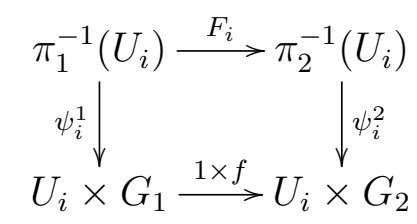

where $\psi_{a}$ are the trivializations which are principal bundle isomorphisms, therefore $\psi_{a}\left(p_{a} g_{a}\right)=$ $\psi_{a}\left(p_{a}\right) g_{a}$, where the action of $G_{a}$ on $U_{i} \times G_{a}$ is given by $\left(x, g_{a}\right) g_{a}^{\prime}=\left(x, g_{a} g_{a}^{\prime}\right)$. From this follows that $F_{i}\left(p_{1} g\right)=F_{i}\left(p_{1}\right) f(g)$. On the other hand, for $p_{1} \in \pi^{-1}\left(U_{i j}\right)$ we have $F_{i}\left(p_{1}\right)=F_{j}\left(p_{1}\right)$. Indeed, we have the commutative diagram for $a=1,2$ :

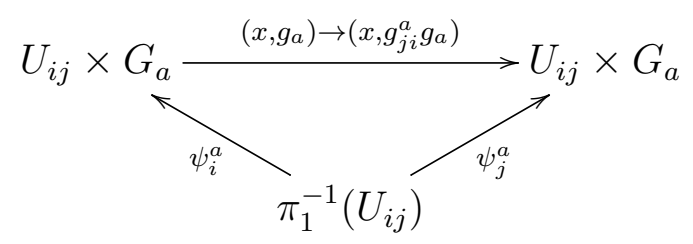


Let $p_{1} \in \pi^{-1}\left(U_{i j}\right)$ and $\psi_{i}^{1}\left(p_{1}\right)=\left(x, g_{1}\right)$, then $\psi_{j}^{1}\left(p_{1}\right)=\left(x, g_{j i}^{1} g_{1}\right)$. Also $\psi_{i}^{2} F_{i}\left(p_{1}\right)=\left(x, f\left(g_{1}\right)\right)$, hence $\psi_{j}^{2} F_{i}\left(p_{1}\right)=\left(x, g_{j i}^{2} f\left(g_{1}\right)\right)$. Therefore,

$$
\psi_{j}^{2} F_{j}\left(p_{1}\right)=\left(x, f\left(g_{j i}^{1} g_{1}\right)\right)=\left(x, f\left(g_{j i}^{1}\right) f\left(g_{1}\right)\right)=\left(x, g_{j i}^{2} f\left(g_{1}\right)\right)=\psi_{j}^{2} F_{i}\left(p_{1}\right),
$$

thus $F_{j}=F_{i}$ on $\pi^{-1}\left(U_{i j}\right)$ and therefore $\left\{F_{i}\right\}$ determine a homomorphism $F: P_{1} \rightarrow P_{2}$.

From the construction of the bundle $\widetilde{P}$ it follows that $P=\widetilde{P} / Z$ and the principal fiber bundle homomorphism constructed in Lemma 3.3.7 is exactly the projection $\widetilde{P} \rightarrow P=\widetilde{P} / Z$.

Let $Q$ be a projective bundle of rank $n, n$ is odd. Assume that $Q$ is orientable. Let $\left\{U_{i}\right\}$ be a good covering of $M, U_{i j}=U_{i} \cap U_{j}$, and $g_{i j}: U_{i j} \rightarrow P G L(n)$ the corresponding cocycle of transition functions. As $U_{i j}$ are contractible, we can choose the maps $\hat{g}_{i j}: U_{i j} \rightarrow S L(n+1)$ such that $p\left(\hat{g}_{i j}\right)=g_{i j}$, where $p: S L(n+1) \rightarrow P G L_{+}(n)$ is the canonical projection. Then $p\left(\hat{g}_{i j} \hat{g}_{j k} \hat{g}_{k i}\right)=g_{i j} g_{j k} g_{k i}=i d \in$ $P G L(n)$, therefore $e_{i j k}=\hat{g}_{i j} \hat{g}_{j k} \hat{g}_{k i}$ takes values in $\mathbb{Z}_{2}=\{ \pm I\}$. Note that $\mathbb{Z}_{2}=\{ \pm I\}$ is the center of the group $S L(n+1)$.

Corollary 3.3.3. If $Q \rightarrow M$ is a projective bundle of odd rank $n$ and is oriented, then the obstruction to existence of a vector bundle $E$ such that $Q=P(E)$ is an element in $H^{2}\left(M, \mathbb{Z}_{2}\right)$.

Definition 3.3.2. Let $Q \rightarrow M$ be a projective bundle of rank $n$. The principal bundle $P G L(Q)$ with the group $P G L(n)$ adjoint to $Q$ is called the bundle of projective frames.

Example 3.3.3. On the total space of a vector bundle $E \rightarrow M$ the group $\mathbb{R}^{*}=\mathbb{R} \backslash\{0\}$ acts freely by scaling, and $Q=P(E)$ is the quotient of $E$ with respect to this action. Therefore, we have the $\mathbb{R}^{*}$-principal bundle $\pi: E \rightarrow Q$. Also we have the induced action of $\mathbb{R}^{*}$ on the total space of the linear frame bundle $G L(E)$, and the quotient space is $P G L(Q)$. The quotient map $G L(E) \rightarrow P G L(Q)$ is $\mathbb{R}^{*}$-principal bundle. If $E$ is an orientable vector bundle of rank $n+1$, where $n$ is odd, then the structure group $G L(n+1)$ of $G L(E)$ reduces to $S L(n+1)$, and in this case we have the double covering $S L(E) \rightarrow P G L(Q)$. For an orientable vector bundle $E$ of rank $n+1$, one can take a metric $g$ on $E$, therefore the $G L(n+1)$-principal bundle $G L(E)$ of linear frames reduces to a $S O(n+1)$ principal bundle of positively oriented orthonormal frames $S O(E)$. Therefore, for $Q=P(E)$, the bundle $P G L(Q)$ reduces to the subgroup $S O(n+1) / \pm I \subset P G L(n)=S L(n+1) / \pm I$.

\section{Projective connections}

Let $Q \rightarrow M$ be a projective bundle. A projective connection is a connection in $P G L(Q)$. Any linear connection in $E$ induces a projective connection in $P(E)$. Indeed, the parallel transport 
preserves one-dimensional subspaces in fibers of $E$. At the same time the canonical projection $G L(E) \rightarrow P G L(P(E))$ is a principal bundle morphism corresponding to the morphism of groups $G L(n+1) \rightarrow P G L(n)$. Therefore, any connection in $G L(E)$ determines a unique connection in $P G L(E)[24]$ (Chapter II, Proposition 1).

Proposition 3.3.4. Two linear connections $\nabla$ and $\nabla^{\prime}$ in the vector bundle $E$ induce the same projective connection in $P(E)$ if and only if the deformation tensor $T=\nabla^{\prime}-\nabla$ has the form

$$
T_{i b}^{a}=\xi_{i} \delta_{b}^{a}
$$

If $Q=P(E)$, where $E$ is an oriented vector bundle, then for any projective connection in $Q$ we can find an equiaffine connection $\nabla$ in $E$ with respect to a given volume form $\theta \in \Omega^{n+1} E$, i. e. $\nabla \theta=0$, such that $\nabla$ induces the given connection in $Q$. Indeed in this case $S L(E, \theta)$ covers $P G L(Q)=S L(E, \theta) / \mathbb{Z}_{2}, \mathbb{Z}_{2}= \pm I$, and any connection in $P G L(P(E))$ lifts to a connection in $S L(E, \theta)$.

Proposition 3.3.5. Let $E$ be an oriented vector bundle, and $\theta \in \Omega^{n+1} E$ be a volume form in $E$. Then there is one-to-one correspondence between equiaffine connections on $E$ with respect to $\theta$ and the projective connections in $P(E)$.

Remark 3.3.3. In terms of parallel translation this correspondence can be explained as follows. Let $\Gamma$ be a projective connection in $Q=P(E)$ induced by a linear connection $\nabla$ in $E$. In terms of the parallel translation this means that a section $L$ of $Q$ along $\gamma$ is parallel with respect to the connection $\Gamma$ if there exists a section $s$ of $E$ along $\gamma$ which is parallel with respect to $\nabla$ and generates $L$. This can be expressed also in terms of covariant derivative: $L=[s]$ is parallel with respect to $\Gamma$ if and only if $\nabla_{\dot{\gamma}} s=\mu s$. Now any projective frame field $\mathcal{L}=\left\{L_{0}, L_{1}, \cdots, L_{n}\right\}$ in $Q=P(E)$ determines a frame field $\mathcal{E}=\left\{E_{1}, \cdots, E_{n}\right\}$ in $E$ such that $L_{i}=\left[E_{i}\right]$, and $L_{0}=\left[E_{0}\right]$, where $E_{0}=E_{1}+E_{2}+\cdots+E_{n}$, up to a scaling. If in addition we have a volume form $\theta$, the projective frame field determines the linear frame field $\left\{E_{i}\right\}$ up to a sign. Then $\nabla_{\dot{\gamma}} E_{i}=\mu_{i} E_{i}$ and $\nabla_{\dot{\gamma}} E_{0}=\mu_{0} E_{0}$, hence follows that $\mu_{0}=\mu_{1}=\cdots=\mu_{n}$. If we assume that the linear connection $\nabla$ is equiaffine with respect to a volume form $\theta$, then $\nabla \theta=0$ and the function $\mu_{0}=\frac{d}{d t} \log \theta\left(E_{1}, \cdots, E_{n}\right)$.

\subsubsection{Section of projective bundle and reduction of the bundle of pro- jective frames}

Proposition 3.3.6. Let $Q$ be a projective bundle of rank 1. Let Aff $(1) \subset P G L(1)$ be the subgroup of affine transformations of line. Then $Q \cong P G L(Q) / A f f(1)$ and a section of projective bundle $Q$ 
is equivalent to the reduction of the bundle $P G L(Q)$ to the subgroup Aff(1).

Proof. This follows from the fact that the group $P G L(1)$ acts transitively on $\mathbb{R} P^{1}$, and the isotropy subgroup of a point in $\mathbb{R} P^{1}$ is the group $\operatorname{Aff}(1)$.

\section{The structure equations of the form $\alpha$}

\section{The vertical form $\alpha$}

Let $E \rightarrow M$ be an oriented vector bundle and $\operatorname{rank}(E)=2$. Let $Q=P(E)$ be the corresponding projective bundle. Also let us take a projective connection $\Gamma$ in $Q$. The typical fiber of $Q$ is $\mathbb{R} P^{1}$. Let us consider the canonical projection $j: \mathbb{R}^{2} \backslash\{0\} \rightarrow \mathbb{R} P^{1}, j\left(v^{1}, v^{2}\right)=\left[v^{1}: v^{2}\right]$, and the corresponding double covering $\bar{\jmath}: \mathbb{S}^{1} \stackrel{\iota}{\hookrightarrow} \mathbb{R}^{2} \backslash\{0\} \stackrel{j}{\rightarrow} \mathbb{R} P^{1}$. Let us consider the form

$$
\theta=\frac{1}{2} \frac{1}{\left(v^{1}\right)^{2}+\left(v^{2}\right)^{2}}\left(-v^{2} d v^{1}+v^{1} d v^{2}\right) \in \Omega^{1}\left(\mathbb{R}^{2} \backslash\{0\}\right),
$$

Then $\iota^{*} \theta$ is the angular form on $\mathbb{S}^{1}$ and there exists $\bar{\alpha} \in \Omega^{1}\left(\mathbb{R} P^{1}\right)$ such that $j^{*} \bar{\alpha}=\theta$. Then, as $\int_{\mathbb{S}^{1}} \iota^{*} \theta=2 \pi$, we have that $\int_{\mathbb{R} P^{1}} \bar{\alpha}=\pi$. Therefore, $\bar{\alpha}$ is a generator of $H^{1}\left(\mathbb{R} P^{1}\right)$. Now let us take a projective connection $H$ is $Q$ which is determined by an equiaffine connection $\hat{H}$ in $E$. Now we can construct the form $\alpha$ in $\Omega^{1}(Q)$ such that $\left.\alpha\right|_{H}=0, d i_{x} \alpha=0$, and $i_{x}^{*} \alpha$ represents the class $[\bar{\alpha}] \in H^{1}\left(\mathbb{R} P^{1}\right)$. Let us take a metric $g$ in $E$, the volume form $\theta$ associated with $g$, and let $\omega$ be the connection form on $E$, that is the projection with kernel $\hat{H}$ onto the vertical subspace. Now let us consider the 1-form

$$
\hat{\alpha}_{v}(X)=\frac{1}{2 g(v, v)} \theta(\omega(X), v) \in \Omega^{1}\left(E_{0}\right),
$$

where $v \in E, E_{0}=E \backslash 0(M)$, and 0 is the zero section of $E$. This form $\hat{\alpha}$ vanishes on $H$, and the metric $g$ allows us to choose the trivialization of the bundle $E$ such that the maps $i_{x}$ are isomorphisms of the euclidean vector spaces $\left(E_{x}, g_{x}\right)$ and $\mathbb{R}^{n}$ with the standard metric. Therefore by $i_{x}$ the form $\hat{\alpha}$ restricted to the fibers is sent to the form (3.32). Now let us consider local coordinate system $\left(x^{i}, y^{a}\right)$ on $E$ such that $g=\left(y^{1}\right)^{2}+\left(y^{2}\right)^{2}$, then $\theta=-y^{2} d y^{1}+y^{1} d y^{2}$. Consider the local coframe $\left\{d x^{i}\right.$, $\left.D y^{a}=d y^{a}+\Gamma_{i b}^{a} y^{b} d x^{i}\right\}$, where $D$ is the differential operator given by (3.11)

\section{Lemma 3.3.7.}

$$
d\left(D y^{a}\right)=\Gamma_{i b}^{a} D y^{b} \wedge d x^{i}+R_{i j b}{ }^{a} y^{b} d x^{i} \wedge d x^{j},
$$

where $R_{i j b}^{a}=\partial_{i} \Gamma_{j b}^{a}-\partial_{j} \Gamma_{i b}^{a}+\Gamma_{i c}^{a} \Gamma_{j b}^{c}-\Gamma_{j c}^{a} \Gamma_{i b}^{c}$ is the curvature tensor of the linear connection in $E$. 
Proof. We have

$$
\begin{gathered}
d\left(D y^{a}\right)=d\left(d y^{a}+\Gamma_{i b}^{a} y^{b} d x^{i}\right)=d \Gamma_{i b}^{a} y^{b} \wedge d x^{i}+\Gamma_{i b}^{a} d y^{b} \wedge d x^{i}= \\
\partial_{j} \Gamma_{i b}^{a} y^{b} d x^{j} \wedge d x^{i}+\Gamma_{i b}^{a}\left(D y^{b}-\Gamma_{j c}^{b} y^{c} d x^{j}\right) \wedge d x^{i}= \\
\Gamma_{i b}^{a} D y^{b} \wedge d x^{i}+\left(\partial_{j} \Gamma_{i b}^{a} y^{b} d x^{j} \wedge d x^{i}-\Gamma_{i b}^{a} \Gamma_{j c}^{b} y^{c} d x^{j} \wedge d x^{i}\right)= \\
\Gamma_{i b}^{a} D y^{b} \wedge d x^{i}+\left(\partial_{j} \Gamma_{i b}^{a} y^{b} d x^{j} \wedge d x^{i}+\Gamma_{j b}^{a} \Gamma_{i c}^{b} y^{c} d x^{j} \wedge d x^{i}\right)= \\
\Gamma_{i b}^{a} D y^{b} \wedge d x^{i}+R_{i j b}^{a} y^{b} d x^{i} \wedge d x^{j}
\end{gathered}
$$

The form $\hat{\alpha}$ is written with respect to the coframe $\left\{d x^{i}, D y^{a}\right\}$ as follows:

$$
\hat{\alpha}=\frac{1}{2} \frac{1}{\left(y^{1}\right)^{2}+\left(y^{2}\right)^{2}}\left(-y^{2} D y^{1}+y^{1} D y^{2}\right) .
$$

Now let us assume that the connection is adapted to the metric $g$ in $E$, in this case $\Gamma_{i b}^{a}=-\Gamma_{i_{a}}^{b}$. Then we have

$$
d\left(\left(y^{1}\right)^{2}+\left(y^{2}\right)^{2}\right)=2\left(y^{1} D y^{1}+y^{2} D y^{2}\right)
$$

because

$$
\begin{aligned}
d\left(\left(y^{1}\right)^{2}+\left(y^{2}\right)^{2}\right)=2\left(y^{1} d y^{1}+y^{2} d y^{2}\right)= & \\
2\left(y^{1}\left(D y^{1}-\Gamma_{i 2}{ }^{1} y^{2} d x^{i}\right)+y^{2}\left(D y^{2}-\Gamma_{i 1}^{2} y^{1} d x^{i}\right)\right) & =2\left(y^{1} D y^{1}+y^{2} D y^{2}\right) .
\end{aligned}
$$

Also we have that

$$
\begin{aligned}
& d y^{1} \wedge D y^{2}=D y^{1} \wedge D y^{2}-\Gamma_{i 2}{ }^{1} y^{2} d x^{i} \wedge D y^{2} \\
& y^{1} d D y^{2}=\Gamma_{i 1}^{2} y^{1} D y^{1} \wedge d x^{i}+R_{i j}{ }_{1}^{2}\left(y^{1}\right)^{2} d x^{i} \wedge d x^{j} \\
& d y^{2} \wedge D y^{1}=D y^{2} \wedge D y^{1}-\Gamma_{i 1}^{2} y^{1} d x^{i} \wedge D y^{1} \\
& y^{2} d D y^{1}=\Gamma_{i 2}^{1} y^{2} D y^{2} \wedge d x^{i}+R_{i j 2}\left(y^{2}\right)^{2} d x^{i} \wedge d x^{j}
\end{aligned}
$$

Therefore,

$$
\begin{aligned}
& d\left(y^{1} D y^{2}-y^{2} D y^{1}\right)= \\
&\left(d y^{1} \wedge D y^{2}+y^{1} d D y^{2}-d y^{2} \wedge D y^{1}-y^{2} d D y^{1}\right)= \\
& 2 D y^{1} \wedge D y^{2}+R_{i j 1}^{2}\left(\left(y^{1}\right)^{2}+\left(y^{2}\right)^{2}\right) d x^{i} \wedge d x^{j} .
\end{aligned}
$$


Thus,

$$
\begin{gathered}
d \hat{\alpha}= \\
\frac{1}{2}\left[d\left(\frac{1}{\left(y^{1}\right)^{2}+\left(y^{2}\right)^{2}}\right) \wedge\left(-y^{2} D y^{1}+y^{1} D y^{2}\right)+\frac{1}{\left(y^{1}\right)^{2}+\left(y^{2}\right)^{2}} d\left(-y^{2} D y^{1}+y^{1} D y^{2}\right)\right]= \\
-\frac{1}{\left(\left(y^{1}\right)^{2}+\left(y^{2}\right)^{2}\right)^{2}}\left(y^{1} D y^{1}+y^{2} D y^{2}\right) \wedge\left(-y^{2} D y^{1}+y^{1} D y^{2}\right)+ \\
+\frac{1}{\left(y^{1}\right)^{2}+\left(y^{2}\right)^{2}} D y^{1} \wedge D y^{2}+\frac{1}{2} R_{i j 1}^{2} d x^{i} \wedge d x^{j} \\
\quad=\frac{1}{2} R_{i j 1}^{2} d x^{i} \wedge d x^{j} .
\end{gathered}
$$

Note that $\Omega=\pi^{*}\left(R_{i j}^{2} d x^{i} \wedge d x^{j}\right)$ is the curvature form of the connection $H$ restricted to $\pi^{-1}(U)$, where $U$ is a coordinate neighborhood and $\left.E\right|_{U}$ is trivial.

Theorem 3.3.8. Let $E \rightarrow M$ be a vector bundle, $Q=P(E)$, and $j: P(E) \rightarrow Q$.

1. There exist a 1-form $\alpha \in \Omega^{1}(Q)$ such that $\hat{\alpha}=j^{*} \alpha$.

2. $\left.\alpha\right|_{H}=0$

3. $d i_{x} \alpha=0$, and $i_{x}^{*} \alpha$ represents the class $[\bar{\alpha}] \in H^{1}\left(\mathbb{R} P^{1}\right)$.

4. $d \alpha=\frac{1}{2} \pi^{*}(\Omega)$.

Theorem 3.3.9. (Gauss-Bonnet-Hopf-Poincaré formula for projective bundles) Let E $\rightarrow$ $M$ be a vector bundle and $Q=P(E)$. Assume that $g$ is a metric in $E$. Let $\Omega$ be the curvature form of the connection in $Q$ induced by a connection in $E$ adapted to $g$. Let $a \in H^{1}\left(\mathbb{R} P^{1}\right)$ is represented by the form $\bar{\alpha}$. If $s: M \backslash \Sigma \rightarrow Q$ is a section with singularities,

$$
\operatorname{ind}(s ; a)=\frac{1}{2} \int_{M} \Omega .
$$

\subsection{Gauss-Bonnet-Hopf-Poincaré formula in $G$-bundles with Singularities}

In the Chapter 2 we introduced the principal bundles with singularities. In this section we give a version of the Gauss-Bonnet-hopf-Poincaré formula in the context of these geometrical structures. To do it we use a given connection on the principal bundle to construct a 1-form on the vertical subbundle of the tangent bundle of a particular fiber bundle associated to the principal bundle, which has the properties given in Proposition 3.2.1. 


\subsubsection{The vertical subbundle of an associated fiber bundle}

Let us recall that there is an important relation between $G$-reductions of a principal $\bar{G}$-bundle $\bar{P} \rightarrow M$ and sections of the fiber bundle $\bar{\pi}: \bar{P} / G \rightarrow M$ which is given by Proposition 1.3.6, and the canonical projection $\pi_{G}: \bar{P} \rightarrow E=\bar{P} / G$ is a principal $G$-bundle [24](Chapter I, Proposition 5.5), and $\pi_{G}$ is a fiber bundle morphism:

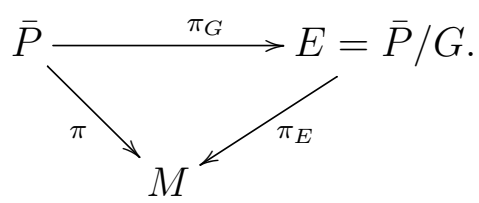

In what follows by $\overline{\mathfrak{g}}$ we denote the Lie algebra of $\bar{G}$, and by $\mathfrak{g}$ the Lie algebra of $G$. Moreover, we suppose that the Lie group $\bar{G}$ is compact and connected. Then $G$ is also compact and the quotient $\bar{G} / G$ is reductive, i.e, $\overline{\mathfrak{g}}$ has an $\operatorname{ad}(G)$-invariant decomposition $\overline{\mathfrak{g}}=\mathfrak{g} \oplus \mathfrak{m}$.

Assume that $\bar{P}$ is endowed with a connection form $\bar{\omega}: T \bar{P} \rightarrow \overline{\mathfrak{g}}$, and let $\bar{H}=\operatorname{ker} \bar{\omega}$ be the horizontal distribution of the connection, and $\bar{V}=\operatorname{ker} d \pi$ the vertical distribution on $\bar{P}$. Note that $\bar{H}$ and $\bar{V}$ are vector subbundles of $T \bar{P}$, and $T \bar{P}=\bar{H} \oplus \bar{V}$.

Define the operator field $\widetilde{\omega}: T \bar{P} \rightarrow \bar{V}$ as follows:

$$
\widetilde{\omega}_{\bar{p}}(\bar{X})=\sigma_{\bar{p}}\left(\bar{\omega}_{\bar{p}}(\bar{X})\right)
$$

where $\bar{X} \in T_{\bar{p}} \bar{P}$, and $\sigma_{\bar{p}}(\bar{a}), \bar{a} \in \bar{g}$, is the value of the fundamental vector field $\sigma(\bar{a})$ at the point $\bar{p}$, i.e.

$$
\sigma_{\bar{p}}(\bar{a})=\left.\frac{d}{d t}\right|_{t=0}[\bar{p} \cdot \exp (\bar{a} t)]
$$

The map $\widetilde{\omega}$ has the properties given in the following lemma.

Lemma 3.4.1. a) $\widetilde{\omega}$ is a field of projectors onto the vertical distribution $\bar{V}$ with kernel $\bar{H}$.

b) $\widetilde{\omega}_{\bar{p} \bar{g}} \circ d R_{\bar{g}}(\bar{X})=d R_{\bar{g}} \circ \widetilde{\omega}_{\bar{p}}(\bar{X})$, where $R_{\bar{g}}: \bar{G} \rightarrow \bar{G}, R_{\bar{g}} \bar{g}^{\prime}=\bar{g}^{\prime} \bar{g}$.

Proof. It is clear that $\widetilde{\omega}$ vanishes on $\bar{H}$. For $\bar{X} \in \bar{V}_{\bar{p}}, \bar{p} \in \bar{P}$, one can find $\bar{a} \in \overline{\mathfrak{g}}$ such that $\sigma_{\bar{p}}(\bar{a})=\bar{X}$. Then, $\bar{\omega}_{\bar{p}}(\bar{X})=\bar{a}$, hence follows that $\widetilde{\omega}_{\bar{p}}(\bar{X})=\sigma_{\bar{p}}(a)=X$. Thus we get a).

Now a) implies b) because $d R_{\bar{g}}: T_{\bar{p}} \bar{P} \rightarrow T_{\bar{p} \bar{g}} P$ is an isomorphism, which maps $\bar{H}_{\bar{p}}$ to $\bar{H}_{\bar{p} \bar{g}}$, and $\bar{V}_{\bar{p}}$ to $\bar{V}_{\bar{p} \bar{g}}$.

Proposition 3.4.2. a) There exists an operator field $\omega_{E}$ on $E$ such that, for each $\bar{p} \in \bar{P}$, the following diagram is commutative:

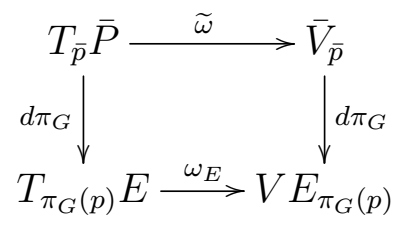

b) $\omega_{E}$ is a projector onto $V E$ with kernel $H E=\operatorname{ker} \omega_{E}=d \pi_{G}(\bar{H})$. 


\subsection{GAUSS-BONNET-HOPF-POINCARÉ FORMULA IN G-BUNDLES WITH SINGULARITIES53}

c) The decomposition $T \bar{P}=\bar{H} \oplus \bar{V}$ of $T \bar{P}$ projects via $d \pi_{G}$ onto the decomposition $T E=H E \oplus V E$ of $T E$

Proof. a) If $d \pi_{G}\left(\bar{X}_{\bar{p}}\right)=d \pi_{G}\left(\bar{X}_{\bar{p}^{\prime}}^{\prime}\right)$, then $\bar{p}^{\prime}=\bar{p} g$, and $\bar{X}^{\prime}=d R_{g} \bar{X}+\bar{Y}$, where $g \in G$ and $\bar{Y}=\sigma_{\bar{p} g}(a) \in$ $\operatorname{ker} d \pi_{G}$ with $a \in \mathfrak{g}$. By Lemma 3.4.1, we have

$$
\widetilde{\omega}_{\bar{p} g}\left(\bar{X}^{\prime}\right)=\widetilde{\omega}_{\bar{p} g}\left(d R_{g} \bar{X}+\sigma_{\bar{p} g}(a)\right)=\widetilde{\omega}_{\bar{p} g}\left(d R_{g} \bar{X}\right)+\sigma_{\bar{p} g}(a)=d R_{g}\left(\widetilde{\omega}_{\bar{p}}(X)+\sigma_{\bar{p} g}(a) .\right.
$$

Then,

$$
d \pi_{G}\left(\widetilde{\omega}_{\bar{p}}(\bar{X})\right)=d \pi_{G}\left(\widetilde{\omega}_{\bar{p}^{\prime}}\left(\bar{X}^{\prime}\right)\right)
$$

this proves that $\omega_{E}$ in the diagram (3.34) is well defined.

b) From the diagram (3.34) it follows that $d \pi_{G}(\bar{H}) \subset H E$. For any $X \in H E_{\pi_{G}(\bar{p})}$, take $\bar{X} \in$ $T_{\pi_{G}(\bar{p})} \bar{P}$ such that $d \pi_{G}(\bar{X})=X$. Then $d \pi_{G}(\widetilde{\omega}(\bar{X}))=0$, this means that $\widetilde{\omega}(\bar{X})=\sigma_{\bar{p}}(a)$, where $a \in \mathfrak{g}$. Then $\widetilde{\omega}\left(\bar{X}-\sigma_{\bar{p}}(a)\right)=0$, therefore $\bar{X}-\sigma_{\bar{p}}(a) \in \bar{H}_{\bar{p}}$ and $d \pi_{G}(\bar{X})-\sigma_{\bar{p}}(a) \in \bar{H}_{\bar{p}}=d \pi_{G}(\bar{X})=X$. Thus $H E \subset d \pi_{G}(\bar{H})$ and therefore $H E=d \pi_{G}(\bar{H})$.

For any $X \in V E_{\pi_{G}(\bar{p})}, X=d \pi_{G}(\bar{X})$, where $\bar{X} \in \bar{V}$. Then, by Lemma 3.4.1 and (3.34), we have that $\omega_{E}(X)=d \pi_{G}(\widetilde{\omega}(\bar{X}))=d \pi_{G}(\bar{X})=X$.

c) follows from $b)$.

Remark 3.4.1. The bundle $E \rightarrow M$ is the bundle associated to the principal $\bar{G}$-bundle $\bar{P} \rightarrow M$ with the fiber $\bar{G} / G$ with respect to the left action of $\bar{G}$ on $\bar{G} / G$. The distribution $H E$ is the connection induced on the associated bundle by the connection $\bar{H}$ on $\bar{P}$.

Lemma 3.4.3. The vertical subbundle $V E$ is isomorphic to the bundle associated to the principal $G$-bundle $\pi_{G}: \bar{P} \rightarrow E$ with the fiber $\mathfrak{m}$ with respect to the left action of $G$ on $\mathfrak{m}: L_{g}(a)=\operatorname{ad}\left(g^{-1}\right) a$.

Proof. The isomorphism $V E \cong \bar{P} \times_{\bar{G}} \bar{G} \times \overline{\mathfrak{g}} / \mathfrak{g} \cong \bar{P} \times_{G} \mathfrak{m}$ follows from the fact that $V E=\bar{P} \times_{\bar{G}}$ $T(\bar{G} / G)[22]($ Chapter IV, section 18.18) and that $T(\bar{G} / G)=\bar{G} \times \overline{\mathfrak{g}} / \mathfrak{g}[23]($ Chapter 4 , section 5). In the exact form the isomorphism is written as follows:

$$
\bar{P} \times_{G} \mathfrak{m} \rightarrow V E, \quad[\bar{p}, a] \mapsto d \pi_{G}\left(\sigma_{\bar{p}}(a)\right)
$$

\subsubsection{The Gauss-Bonnet-Hopf-Poincaré formula for principal $G$-bundle with singularities}

Let $M$ be a 2-dimensional compact connected oriented manifold. Let $\bar{P}(M, \bar{G}, \bar{\pi})$ be a principal $G$-bundle with singularities, where the set of singularities $\Sigma$ consists of isolated points, and $\bar{G}$ is a compact Lie group. Consider the fiber bundle $\pi_{E}: E=\bar{P} / G \rightarrow M$. Let us take a connection $\bar{H}$ in 
$\bar{P}$ with the corresponding connection form $\bar{\omega}$. Let $E=\bar{P} / G$ and $H$ be the connection in $E$ induced by $\bar{H}$ with the connection form $\omega_{E}$ (see Proposition 3.4.2 and Remark 3.4.1).

Given a 1-cohomology class $a \in H^{1}(\bar{G} / G)$, by Proposition 3.2.1 we can construct a 1-form $\alpha \in \Omega^{1}(E)$ on $E$ such that

1. $\left.\alpha\right|_{H}=0$;

2. for each $x \in M, d i_{x}^{*} \alpha=0$ and $\left[i_{x}^{*} \alpha\right]=H^{1}\left(\eta_{x}\right) a$.

In this case one can construct the form $\alpha$ explicitly. As $\bar{G} / G$ is a homogeneous space of a compact Lie group and $\overline{\mathfrak{g}}=\mathfrak{g} \oplus \mathfrak{m}$ is the $\operatorname{Ad}(G)$-invariant decomposition of $\overline{\mathfrak{g}}$, we can take an invariant form $\xi \in \Omega_{\text {inv }}^{1}(\bar{G} / G) \cong \Lambda^{1}(\mathfrak{m})$ such that $[\xi]=a$ (see, e.g., [3], Ch.1, 1 ). Note that $\xi$ is determined by its value $\xi_{0}: \mathfrak{m} \rightarrow \mathbb{R}$ at $[e] \in \bar{G} / G$. Then from Lemma 3.4.3 it follows that $\xi$ defines a form $\widetilde{\alpha} \in \Omega^{1}(V E)$ :

$$
\widetilde{\alpha}([\bar{p}, a])=\xi_{0}(a) .
$$

Theorem 3.4.4. Let $\omega_{E}: T E \rightarrow V E$ be a connection form induced by a connection in the principal $\bar{G}$-bundle $\bar{P} \rightarrow M$ (see Proposition 3.4.2). Then the form given by

$$
\alpha(X)=\tilde{\alpha}\left(\omega_{E}(X)\right)
$$

satisfies the properties (1) and (2) of Proposition 3.2.1.

Proof. For $X \in H, \omega_{E}(X)=0$, therefore $\alpha(X)=0$.

Recall that the trivializations of $E=P / G$ are constructed in the following way. For a good covering $U_{i}$ of $M$ we take sections $s_{i}: U_{i} \rightarrow P$, then we define the charts

$$
\psi_{i}: U_{i} \times \bar{G} / G \rightarrow \pi_{E}^{-1}\left(U_{i}\right), \quad(x,[\bar{g}]) \rightarrow\left[s_{i}(x) \bar{g}\right]=\pi_{G}\left(s_{i}(x) \bar{g}\right) .
$$

Therefore, for $x \in U_{i}, \eta_{x}^{-1}: \bar{G} / G \rightarrow E_{x}=\pi_{E}^{-1}(x), \eta_{x}^{-1}[\bar{g}]=\pi_{G}\left(s_{i}(x) \bar{g}\right)$.

Now let us fix $x \in U_{i} \subset M$ and let $\bar{p}=s_{i}(x)$. For any $y \in E_{x}$ and $Y \in V_{y} E$, we have that $y=\pi_{G}(\bar{p} \bar{g})=[\bar{p}, \bar{g}]=\eta_{x}^{-1}(\bar{g})$ and $Y=d \pi_{G}\left(\sigma_{\bar{p} \bar{g}}(a)\right)=[\bar{p} \bar{g}, a]=d \eta_{x}^{-1}\left(d L_{\bar{g}} a\right)$, where $a \in \mathfrak{m}$, and $L_{\bar{g}}: \bar{G} / G \rightarrow \bar{G} / G, L_{\bar{g}} \bar{g}^{\prime}=\bar{g} \bar{g}^{\prime}$. Note that here we use the isomorphism constructed in Lemma 3.4.3. Then

$$
\alpha(Y)=\widetilde{\alpha}\left(\omega_{E}(Y)\right)=\widetilde{\alpha}(Y)=\xi_{0}(a)=\xi\left(d L_{\bar{g}} a\right) .
$$

Therefore $\alpha\left(d \eta_{x}^{-1}\left(d L_{\bar{g}} a\right)\right)=\xi\left(d L_{\bar{g}} a\right)$, thus $\left.\left(\eta_{x}^{-1}\right)^{*} \alpha\right|_{E_{x}}=\xi$, then $\eta_{x}^{*} \xi=\left.\alpha\right|_{E_{x}}$.

Thus $d\left(\left.\alpha\right|_{E_{x}}\right)=0$, and $\left[\left.\alpha\right|_{E_{x}}\right]=H^{1}\left(\eta_{x}\right) a$.

The curvature form $\Omega_{E}=D \omega_{E}$ of the connection $\omega_{E}$ (see Section 3.2, equation (3.2.4)) can be represented by the following equality:

$$
\Omega_{E}=1 / 2\left[\omega_{E}, \omega_{E}\right],
$$




\subsection{GAUSS-BONNET-HOPF-POINCARÉ FORMULA IN G-BUNDLES WITH SINGULARITIES55}

where [, ] : $\Omega^{k}(E, T E) \times \Omega^{l}(E, T E) \rightarrow \Omega^{k+l}(E, T E)$ is the Frolicher-Nijenhuis bracket [22](Chapter IV, section 16.3). In particular, for 1 -forms $\omega_{1}$ and $\omega_{2}$ this bracket is given by the following equation

$$
\begin{aligned}
{\left[\omega_{1}, \omega_{2}\right](X, Y) } & =\left[\omega_{1}(X), \omega_{2}(Y)\right]-\left[\omega_{1}(Y), \omega_{2}(X)\right]-\omega_{2}\left(\left[\omega_{1}(X), Y\right]\right) \\
& \omega_{1}\left(\left[\omega_{2}(X), Y\right]\right)-\omega_{2}\left[\omega_{1}(Y), X\right]-\omega_{1}\left[\omega_{2}(Y), X\right]+\left(\omega_{1} \circ \omega_{2}+\omega_{2} \circ \omega_{1}\right)[X, Y] .
\end{aligned}
$$

We repeat the arguments of subsection 3.2.4 and get that

$$
d \alpha=L_{X} \alpha+1 / 2 \tilde{\alpha}\left(\left[\omega_{E}, \omega_{E}\right]\right) .
$$

Thus, by assuming the existence of the 2-dimensional oriented compact manifold $S$ and the map $q: S \rightarrow E$ satisfying the properties given in Section 3.5, by Theorem 3.2.7, we obtain the following equality

$$
\int_{S}\left(q^{*} L_{X} \alpha+\frac{1}{2} q^{*} \tilde{\alpha}\left(\left[\omega_{E}, \omega_{E}\right]\right)\right)=\sum_{i=\overline{1, k}} \operatorname{index}_{x_{i}}(s, a),
$$

where $s: M \backslash \Sigma \rightarrow E$ is the section corresponding to the reduction of $\left.\bar{P}\right|_{M \backslash \Sigma}$ to $G$. The result given by the equation (3.36) will be called the Hopf-Gauss-Bonnet-Hoph-Poincaré formula for principal G-bundle with singularities.

Remark 3.4.2. If $X$ is a vector field on $M$, and $\Sigma$ is the set of zeros of $X$, then $Y=\frac{X}{|X|}$ defines a section of the unit tangent vectors bundle $M \rightarrow T^{1} M$ over $M \backslash \Sigma$. Since $T^{1} M$ is the associated bundle to the bundle of orthonormal frames $M \rightarrow S O(M)$ with respect of standard action of $S O(2)$ on $\mathbb{S}^{1}$, the bundle $\left.S O(M)\right|_{M \backslash \Sigma}$ reduces to the trivial subgroup $G=\{e\}$ of $\bar{G}=S O(2)$. Now, $\bar{G} / G=S O(2)$, and $S O(2)$ is diffeomorphic to $\mathbb{S}^{1}$, then $H^{1}(\bar{G} / G)$ is 1-dimensional vector space generated by the angle form on $\mathbb{S}^{1}$. Therefore, we can choose this generator to construct the form $\alpha \in \Omega^{1}(S O(M))$, and the connection form on $S O(M)$ induced by the Levi-Civita connection. This connection form is given by equation (3.23). Thus, if $S$ and $q: S \rightarrow \mathbb{S}^{1} M$ are the manifold and the map, respectively, constructed in section 3.3, the equation (3.36) reduces to the equation (3.31). Hence, Gauss-BonnetHopf-Poincaré formula principal $G$-bundle with singularities is a generalization of the classical GaussBonnet theorem.

\subsubsection{Hopf-Gauss-Bonnet-Hopf-Poincaré formula for the case of the sum of Whitney of vector bundles}

Let $E_{1}, E_{2}, \cdots, E_{k}$ be $k$ vector bundles of rank 2 over a compact connected Riemannian twodimensional manifold $M$, and for each $i$, let $s_{i}^{0}: M \rightarrow E_{i}$ be the zero section of $E_{i}$. Let $\tilde{E}_{i}=$ $E_{i} \backslash s_{i}^{0}(M)$. 
For all $i$ let us take sections $s_{i}: M \rightarrow E_{i}$. Then $s_{i}$ is a section with singularities of $\tilde{E}_{i}$, whose set of singularities is the set $\Sigma_{i}$ of zeros of $s_{i}$. Thus, if we consider the product bundle $\tilde{E}=\tilde{E}_{1} \times \tilde{E}_{2} \times \cdots \times \tilde{E}_{k}$ over $M \backslash \Sigma$, where $\Sigma=\cup \Sigma_{i}$, then the map $s=\left(s_{1}, \cdots, s_{k}\right): M \backslash \Sigma \rightarrow \tilde{E}$ is a section with singularities of $\tilde{E}$. In addition, suppose that the section $s: M \backslash \Sigma \rightarrow \tilde{E}$ admits a resolution of the singularities (see section 3.5). This means that there exists a 2-dimensional oriented compact manifold $S$ with boundary $\partial S=\bigcup_{j=\overline{1, k}} S_{j}$, and a map $q: S \rightarrow \tilde{E}$ such that $q$ restricted to $\stackrel{\circ}{S}=S \backslash \partial S$ is a diffeomorphism between $S \backslash \partial S$ and $s(M \backslash \Sigma)$.

Let us take connection forms $\omega_{i}: T \tilde{E}_{i} \rightarrow V \tilde{E}_{i}$ on $\tilde{E}_{i}$, for $i=1, \cdots, k$, then $\omega=\left(\omega_{1}, \cdots, \omega_{k}\right)$ is a connection form on $\tilde{E}$. The curvature form of $\omega$ is $\Omega=\left(\Omega_{1}, \cdots, \Omega_{k}\right)$, where $\Omega_{i}$ is the curvature form of $\omega_{i}$.

Since the fiber of the bundle $\tilde{E}_{i}$ is $F_{i}=\mathbb{R}^{2} \backslash\{(0,0)\}$, and the cohomology class $a_{i}=\left[d \phi_{i}\right]$ represented by the angular form on $F_{i}$ is a generator of $H^{1}\left(F_{i}\right)$, the 1 -form $\left(d \phi_{1}, \cdots, d \phi_{k}\right)$ represents a nonzero cohomology class $a \in H^{1}(F)$, where $F=F_{1} \times \cdots \times F_{k}$ is the fiber of the bundle $\tilde{E}$. Note that $F$ retracts by deformation to the $k$-torus $\mathbb{T}^{k}$. With this in mind, we can choose the 1 -form $\alpha \in \Omega^{1}(E)$ satisfying properties (1) and (2) of Proposition 3.2.1 as follows:

$$
\alpha=\omega_{1}+\cdots+\omega_{k}
$$

where $\omega_{i}$ is the connection form of $\tilde{E}_{i}$.

Then $d \alpha=\pi^{*} d G$, where $G=\sum_{i=1}^{k} G_{i}$, and by applying Theorem 3.2.7 we obtain that

$$
\int_{S} q^{*} \pi^{*} d G=\sum_{x_{i} \in \Sigma} \operatorname{index}_{x_{i}}(s, a) .
$$

Let us fix an area form $\theta$ on $M$. Let $d G_{i}=K_{i} \theta$, and call the function $K_{i}$ the curvature of the connection $\omega_{i}$.

If we consider the points of $\Sigma \backslash \Sigma_{i}$ as singular points of the section $s_{i}$, then it is a section with singularities of the bundle $\tilde{E}_{i} \rightarrow M$. In this sense we have the following proposition.

Theorem 3.4.5. Let $M$ be a compact connected two-dimensional manifold, let $E_{i} \rightarrow M, i=1, \cdots, k$, be $k$ vector bundles of rank 2 over $M$, let $\tilde{E}_{i}=E_{i} \backslash s_{i}^{0}(M)$, where $s_{i}^{0}: M \rightarrow E_{i}$ is the zero section of $E_{i}$, and let $s_{i}: M \backslash \Sigma_{i} \rightarrow E_{i}$ be a section of $E_{i}$ for each $i$. If $\omega_{1}, \cdots, \omega_{k}$ are connections on $E_{1}, \cdots, E_{k}$ respectively, $K_{1}, \cdots, K_{k}$ are the curvatures of these connections, and $s=\left(s_{1}, \cdots, s_{k}\right): M \backslash \Sigma \rightarrow \tilde{E}$, then

$$
\sum_{j=\overline{1, k}} \int_{M} K_{i} \theta=\sum_{x_{i} \in \Sigma} \sum_{j=\overline{1, k}} i n d_{x_{i}}\left(s_{j},\left[d \phi_{j}\right]\right),
$$

where $d \phi_{i} \in \Omega^{1}\left(\mathbb{R}^{2}-\{(0,0)\}\right)$ is the angular form of $\mathbb{R}^{2}-\{(0,0)\}$, and $\theta$ is the area form on $M$. 
Proof. Let $S$ be an oriented 2-manifold with boundary, and let $q: S \rightarrow E$ be a map which satisfies the condition given in section 3.5, that is, $q_{i}$ restricted to $\stackrel{\circ}{S}=S \backslash \partial S$ is a diffeomorphism between $S \backslash \partial S$ and $s_{i}(M \backslash \Sigma)$. Now, because $E=\tilde{E}_{1} \times \cdots \tilde{E}_{k}, q$ has the form $q=\left(q_{1}, \cdots, q_{k}\right)$, and for each $i$, the map $q_{i}: S \rightarrow \tilde{E}_{i}$ also satisfies this condition. This fact follows from the commutative diagram

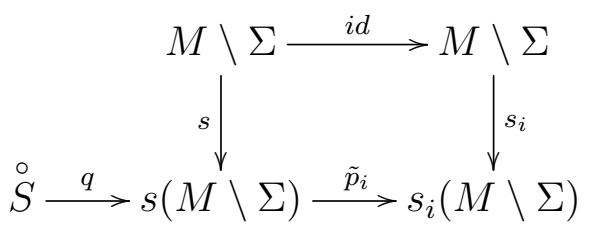

Since the vertical arrows are diffeomorphisms, we have that $\tilde{p}_{i}$ is a diffeomorphism. Therefore, for each $i=1, \cdots, k$, the map $q_{i}=\tilde{p}_{i} \circ q$ is also a diffeomorphism.

Let us take the cohomology class $a=\left[\left(d \phi_{1}, \cdots, d \phi_{k}\right)\right]$ of the fiber of $\tilde{E}$, where $d \phi_{i}$ is the angular form on the fiber $F_{i}$ of $E_{i}$, then $a=\left[d \phi_{1}\right]+\cdots+\left[d \phi_{k}\right]$. Thus, the expression at the right side of equation (3.37) can be written as follows

$$
\sum_{x_{i} \in \Sigma} \operatorname{index}_{x_{i}}(s, a)=\sum_{x_{i} \in \Sigma} \sum_{j=\overline{1, k}} \operatorname{ind}_{x_{i}}\left(s_{j},\left[d \phi_{j}\right]\right) .
$$

Furthermore, $d G_{i}=K_{i} \theta$, and $d \alpha=\sum_{j=1, k} \pi^{*}\left(K_{i} \theta\right)$. Therefore, the expression at the left hand side of equation (3.37) reduces to the following equality

$$
\int_{S} q^{*} \pi^{*} d G=\sum_{j=\overline{1, k}} \int_{M} K_{i} \theta
$$

Hence, we obtain the following equation

$$
\sum_{i=\overline{1, k}} \int_{M} K_{i} \theta=\sum_{x_{i} \in \Sigma} \sum_{j=\overline{1, k}} i n d_{x_{i}}\left(s_{j},\left[d \phi_{j}\right]\right)
$$

Remark 3.4.3. Note that if $\Sigma_{i} \cap \Sigma_{j}$ is non empty, and $x \in \Sigma_{i} \cap \Sigma_{j}$, then the indexes $\operatorname{ind}_{x}\left(s_{i}\right)$ and $\operatorname{ind}_{x}\left(s_{j}\right)$ could be different, still in the case where the vector bundles $E_{i}$ and $E_{j}$ are the same.

\subsection{The Gauss-Bonnet-Hopf-Poincaré formula to branched sections.}

In this section we define the index of a singular point of a branched section, and give examples of its calculation, in particular for branched sections of the projective tangent bundle of $M$ determined 
by binary differential equations. Also we define a resolution of singularities of a branched section, and prove an analog of Gauss-Bonnet-Hopf-Poincaré formula for the branched sections admitting a resolution.

\subsubsection{Branched sections}

Let $\xi=\{\pi: E \rightarrow M\}$ be a locally trivial fiber bundle, let $\Sigma$ be a closed subset of $M, M^{\prime}=M \backslash \Sigma$, and $E^{\prime}=\pi^{-1}\left(M^{\prime}\right)$.

Definition 3.5.1. A $k$-sheeted branched section of the bundle $\xi$ is a subset $Q \subset E$ such that $Q^{\prime}=Q \cap E^{\prime}$ is an embedded submanifold of $E$ and $\left.\pi\right|_{Q^{\prime}}: Q^{\prime} \rightarrow M^{\prime}$ is a $k$-sheeted covering. The set $\Sigma$ is called the singularity set of the branched section $Q$. The set $\pi(Q)$ is called the domain of the branched section $Q$.

Example 3.5.1. If $V$ is a vector field on a manifold $N$, that is a section of the tangent bundle $\pi_{T N}: T N \rightarrow N$, and $f: N \rightarrow M$ is a $k$-sheeted covering, then generally $V$ does not define a section $d f(V)$ of the bundle $\pi_{T M}: T M \rightarrow M$, however it defines a branched section of this bundle.

Indeed, let us consider the subset $Q=\left\{d f_{y}(V(y)) \mid y \in N\right\} \subset T M$. For each $x \in M$, let us set $\mathcal{V}(x)=\left\{d f_{y}(V(y)) \mid y \in f^{-1}(x)\right\} \subset T_{x} M$. Take the subset $\Sigma \subset M$ consisting of points $x \in M$ such that the number of elements of the set $\mathcal{V}(x)$ is less than $k$. Then $M^{\prime}=M \backslash \Sigma$ is open, $Q^{\prime}=Q \cap \pi_{T M}^{-1}\left(M^{\prime}\right)$ is a submanifold of $T M$ and $f$ induces a $k$-sheeted covering $f^{\prime}: Q^{\prime} \rightarrow M^{\prime}$. Indeed, for each $x \in M^{\prime}$ there exists a neighborhood $U$ of $x$ such that $f^{-1}(U)=\bigsqcup_{j=1}^{k} \widetilde{U}_{j}$ and for each $j$ the application $f_{j}=\left.f\right|_{\widetilde{U}_{j}}: \widetilde{U}_{j} \rightarrow U$ is a diffeomorphism, therefore $d f_{j}: T \widetilde{U}_{j} \subset T N \rightarrow T U \subset T M$ is a diffeomorphism, as well. At the same time, $V: \widetilde{U}_{j} \rightarrow V\left(\widetilde{U}_{j}\right) \subset T N$ is a diffeomorphism onto its image. Therefore the map $\theta_{j}=d f_{j} \circ V \circ f_{j}^{-1}: U \rightarrow d f_{j}\left(V\left(\widetilde{U}_{j}\right)\right) \subset Q \subset T M, j=\overline{1, k}$, is a diffeomorphism onto its image, and $\theta_{i}(U) \cap \theta_{j}(U)=\emptyset$, for $i \neq j$, by the definition of $M^{\prime}$. Thus $\pi_{T M}^{-1}(U) \cap Q^{\prime}=\bigsqcup_{j=1}^{k} \theta_{j}(U)$, this means that $U$ is simply covered in $Q^{\prime}$, and $Q^{\prime}$ is a $k$-sheeted covering of $M^{\prime}$.

Example 3.5.2. Let $M$ be a connected compact oriented manifold and let $\omega$ be a symmetric tensor of order $n$ over $M$. Recall that such a tensor can be written locally as follows

$$
\omega_{(x, y)}=a_{0}(x, y) d x^{n}+a_{1}(x, y) d x^{n-1} d y+\cdots+a_{n}(x, y) d y^{n}
$$

where $(x, y)$ are coordinate functions on an open set $U \subset M$, and $a_{i}: U \rightarrow \mathbb{R}$ are smooth functions defined in $U$. In what follows, we suppose that $\omega$ has the following properties: 
1. The function $\omega_{(x, y)}$ is identically zero if and only if $a_{i}(x, y)=0$ for $0 \leq i \leq n$. We set $\Sigma=\left\{p \in M: \omega_{p}=0\right\}$.

2. On $M \backslash \Sigma$, the tensor $\omega$ has the form $\omega=\lambda_{1} \lambda_{2} \cdots \lambda_{n}$, where $\lambda_{i} \in \Omega(M \backslash \Sigma)$ pairwise linearly independent.

Proposition 3.5.1. The $n$-form $\omega$ determines a branched section of the bundle $\pi:$ PTM $\rightarrow M$

Proof. Let $Q$ be the solution on PTM of the equation $\omega=0$, where $\omega$ is given by (3.38). We will prove that $Q$ is a branched section of $\pi$. Let $E^{\prime}=\pi^{-1}(M \backslash \Sigma)$ and $Q^{\prime}=Q \cap E^{\prime}$. It follows from the property (2) that the set $F_{p}=Q \cap \pi^{-1}(p), p \in M \backslash \Sigma$ has exactly $n$ elements, therefore each fiber of the surjective map $\pi^{\prime}:=\left.\pi\right|_{Q^{\prime}}: Q^{\prime} \rightarrow M \backslash \Sigma$ is finite with $n$ elements. On the other hand, if $\varphi: \pi^{-1}(U) \rightarrow U \times \mathbb{R} P^{1}$ is a trivialization of $P T M$ on $U$, then the restriction $\varphi^{\prime}:=\left.\varphi\right|_{\pi^{-1}(U) \cap Q^{\prime}}$ : $\pi^{-1}(U) \cap Q^{\prime} \rightarrow U \times \mathbb{R} P^{1}$ is a homeomorphism onto its image. Since $\left.\pi\right|_{\pi^{-1}(U) \cap Q^{\prime}}: \pi^{-1}(U) \cap Q^{\prime} \rightarrow$ $U \cap(M \backslash \Sigma)$ has finite fiber with $n$ elements over each point $p \in U \cap(M \backslash \Sigma)$, from the following commutative diagram

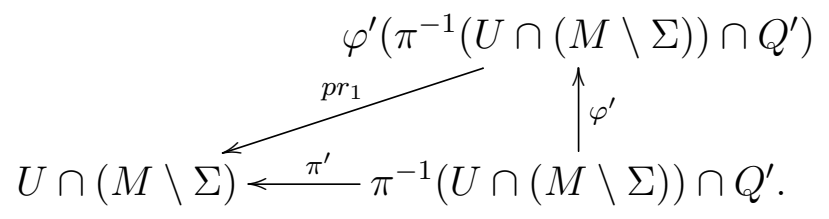

It follows that $\left.\pi\right|_{Q^{\prime}}: Q^{\prime} \rightarrow M \backslash \Sigma$ is a local diffeomorphism. Therefore, $\left.\pi\right|_{Q^{\prime}}: Q^{\prime} \rightarrow M \backslash \Sigma$ is a $n$-sheeted branched covering, and so $Q$ is a branched section of PTM.

Geometrically $Q$ determines an $n$-web at the points of $M \backslash \Sigma$.

Example 3.5.3. Let $\xi=\{\pi: \bar{P} \rightarrow M\}$ be a $\bar{G}$-principal bundle which reduces to a finite subgroup $G \subset \bar{G}$ over $M \backslash \Sigma$, where $\Sigma \subset M$ is a closed subset. Then the corresponding $G$-principal bundle $P \subset \bar{P}$ is a branched section of the bundle $\xi$ with singularity set $\Sigma$.

For example, let $M$ be a two-dimensional oriented Riemannian manifold, and $\bar{P}=S O(M)$ be the $S O(2)$-principal bundle of orthonormal positively oriented frames of $M$. Any finite subgroup $G \subset S O(2)$ is a cyclic group $G \cong \mathbb{Z}_{m}$ generated by the rotation $R_{2 \pi / m}$.

If $P \subset S O\left(M^{\prime}\right) \subset S O(M)$ is a reduction of $S O(M)$ to $G$ over $M^{\prime}=M \backslash \Sigma$, then at each point $x \in M^{\prime}$ we have the set $\mathcal{N}(x)=\left\{e \in T_{x} M \mid\left(e, R_{\pi / 2} e\right) \in P\right\}$, which consists of $m$ unit vectors such that the angle between any two of them is $2 \pi l / m$. The set $\mathcal{N}(x)$ defines a regular $m$-polygon $P_{m} \subset T_{x} M^{\prime}$ inscribed in a unit circle centered at $0 \in T_{x} M$.

It is clear that, vice versa, if at any point of $M^{\prime}=M \backslash \Sigma$, we are given a unitary $m$-polygon $P_{m} \subset T_{p} M^{\prime}$ and the field of these polygons is smooth (these means that locally we can choose $m$ 
unitary vector fields whose values are the vertices of the polygons $\left.P_{m}\right)$, then the bundle $S O(M)$ reduces to the subgroup $G \cong \mathbb{Z}_{m}$ of the Lie group $S O(2)$.

This situation occurs, for example, when $M$ is a surface in $\mathbb{R}^{3}$, and $\Sigma$ is the set of umbilic points of $M$. Then at each point of $M^{\prime}$ we have two orthogonal eigenspaces of the shape operator of the surface, which determine a square in $T_{p} M$ with vertices at points where these eigenspaces meet the unit circle centered at $0 \in T_{p} M$. Therefore, over $M^{\prime}=M \backslash \Sigma$ the bundle $S O(M)$ reduces to the subgroup $G \cong \mathbb{Z}_{4}$ generated by the rotation $R_{\pi / 2}$. The corresponding principal subbundle $P$, the branched section of the bundle $S O(M) \rightarrow M$, consists of oriented orthogonal frames such that the frame vectors span the eigenspaces.

Moreover, as the difference of the principal curvatures never vanish on $M^{\prime}$, we can order the principal curvatures in such a way that $k_{1}(p)>k_{2}(p)$ at any $p \in M^{\prime}$. Let $L_{a}(p), a=1,2$ be the eigenspace corresponding to the principal curvature $k_{a}(p), a=1,2$. Then we can choose the subbundle $P \subset S O(M)$ in such a way that, for $\left\{e_{1}, e_{2}\right\} \in P$, the vector $e_{a}$ spans $L_{a}, a=1,2$, therefore in this case the bundle $S O(M) \rightarrow M$ reduces to the group $G \cong \mathbb{Z}_{2}$.

Also, note that this example is related to Example 3.5.2. Indeed if we have the reduction of $P \subset S O(M)$ to the subgroup $G \cong \mathbb{Z}_{m}$ over $M^{\prime}$, then at each point $p \in M^{\prime}$ we have $m$ (or $\left.m / 2\right)$ subspaces spanned by the vector $e_{1}$ from the frame $\left\{e_{1}, e_{2}\right\} \in P$. Then we can take the binary differential equation (3.38) such that these subspaces are the roots of the corresponding algebraic equation.

\subsection{The index of a singular isolated point}

\subsubsection{Local monodromy group}

Let $M$ be a two-dimensional closed oriented manifold. Let $\xi=\left\{\pi_{E}: E \rightarrow M\right\}$ be a fiber bundle with oriented typical fiber $F$.

Let us consider a $k$-sheeted branched section $Q$ of $\xi$ (see Definition 3.5.1) with singularity set $\Sigma$, and let $\pi_{Q}=\left.\pi_{E}\right|_{Q}: Q \rightarrow M$. Recall that $M^{\prime}=M \backslash \Sigma, E^{\prime}=\pi^{-1}\left(M^{\prime}\right)$, and $Q^{\prime}=Q \cap E^{\prime}$.

Assume that $x \in \Sigma$ is an isolated point of $\Sigma$. Let us take a neighborhood $U(x)$ such that $U^{\prime}(x)=U(x) \backslash\{x\}$ is an open subset of $M^{\prime}$ and there exists a diffeomorphism $\varphi:(D, 0) \rightarrow(U(x), x)$, where $D \subset \mathbb{R}^{2}$ is the standard open 2-disk centered at the origin $0 \in \mathbb{R}^{2}$. We will call $U(x)$ a disk neighborhood of $x$ and assume that $\varphi$ sends the standard orientation of $D$ to the orientation of $U(x)$ induced by the orientation of $M$.

By Definition 3.5.1, the map $\left.\pi_{Q}\right|_{\pi_{Q}^{-1}\left(U^{\prime}(x)\right)}: \pi_{Q}^{-1}\left(U^{\prime}(x)\right) \rightarrow U^{\prime}(x)$ is a $k$-sheeted covering. 
If $U(x)$ is a disk neighborhood of an isolated point $x \in \Sigma$, then for each point $y \in U^{\prime}(x)$, the fundamental group $\Pi_{1}(y)=\pi_{1}\left(U^{\prime}(x), y\right)$ is isomorphic to $\mathbb{Z}$. There are two generators of $\Pi_{1}(y)$ : $\left[\gamma_{+}\right]$ and $\left[\gamma_{-}\right]$, where $\gamma_{ \pm}=\phi\left(C_{ \pm}\right)$and $C_{ \pm}$is a circle in $D$ passing through the point $\varphi^{-1}(y)$ and enclosing the origin, and having positive (negative, respectively) orientation. We will call the element $\left[\gamma_{ \pm}\right] \in \Pi_{1}(y)$ the positive (the negative, respectively) generator of $\Pi_{1}(y)$.

The group $\Pi_{1}(y)$ acts on the fiber $Q_{y}=\pi_{Q}^{-1}(y)$ in the following way: for an element $[\gamma] \in \Pi_{1}(y)$ and $q \in Q_{y}$ we set $[\gamma] \cdot q=\bar{q}$ if the lift $\widetilde{\gamma}$ of $\gamma$ starting at $q$ terminates in $\bar{q}$. This action is well defined, this means that if $\gamma_{1}$ and $\gamma_{2}$ represent the same element in $\Pi_{1}(y)$, then the lifts $\widetilde{\gamma}_{1}$ and $\widetilde{\gamma}_{2}$ starting at a same point $q$ terminate at a same point $\bar{q}$.

This action is a homomorphism of the group $\Pi_{1}(y)$ to the group of permutations of the fiber $Q_{y}$ and its image is called the local monodromy group of the branched section $Q$ at the point $y \in M^{\prime}$.

Proposition 3.6.1. The local monodromy group does not depend on a choice of the disk neighborhood $U(x)$.

Proof. Let $U(x)$ and $V(x)$ be two disk neighborhoods of $x$, and $y$ lies in $U(x) \cap V(x)$. Then $\Pi_{1}^{U}(y)=$ $\pi_{1}\left(U^{\prime}(x), y\right)=\Pi_{1}^{V}(y)=\pi_{1}\left(V^{\prime}(x), y\right)$ because for each class $[\gamma] \in \pi_{1}\left(V^{\prime}(x), y\right)$ or $[\gamma] \in \pi_{1}\left(U^{\prime}(x), y\right)$ one can find a representative $\gamma_{1} \in[\gamma]$ which takes values in $U^{\prime}(x) \cap V^{\prime}(x)$.

Proposition 3.6.2. Let $\gamma$ be a loop in $U^{\prime}(x)$ based at a point $y \in U^{\prime}(x)$ such that its homotopic class represents the positive generator of $\Pi_{1}(y)$. Then for each orbit $O$ of the local monodromy group action on $Q_{y}$ and each point $q \in O$, there exists a loop $\widetilde{\gamma}$ in $\pi_{Q}^{-1}\left(U^{\prime}(x)\right)$ based at $q$ which passes through each point of the orbit once and only once and such that $\pi_{1}\left(\pi_{E}\right)([\widetilde{\gamma}])=[\gamma]^{k}$, where $k$ is the number \#O of elements of the orbit $O$. Here $\pi_{1}\left(\pi_{E}\right): \pi_{1}\left(\pi_{Q}^{-1}\left(U^{\prime}(x)\right), q\right) \rightarrow \pi_{1}(U(x), y)$ is the homomorphism of the fundamental groups induced by the map $\pi_{E}$.

Proof. First of all note that if we have an action of the group $\mathbb{Z}$ on a finite set, then we can enumerate elements of each orbit in such a way that the action of the group generator 1 on this orbit is represented by the cycle $\sigma=(2,3, \cdots, 1)$. Indeed, let $O$ be an orbit of the action, and $q \in O$. The map $F: \mathbb{Z} / H_{q} \rightarrow O,[g] \rightarrow g \cdot q$, where $H_{q}$ is the isotropy subgroup of the action, is an equivariant bijection. The group $H_{q}$ is a cyclic group, this means that there exists $k \in \mathbb{Z}, k \geq 0$ such that $H_{q}=\{k m \mid m \in \mathbb{Z}\}$, therefore $\mathbb{Z} / H_{q}=\{[0],[1], \cdots,[k-1]\}$, and the action of the generator $1 \in \mathbb{Z}$ on $\mathbb{Z} / H_{p}$ is given exactly by the cycle $\sigma$.

Now, for a point $y \in U^{\prime}(x)$, let $[\gamma], \gamma:[0,1] \rightarrow U^{\prime}(x)$, be the positive generator of $\Pi_{1}(y)$. Let us take an orbit $O$ of the local monodromy group action on $Q_{y}$ and a point $q \in O$. Let $k$ be the number of elements of $O$. As we have seen, the action of $[\gamma]$ on $O$ is represented by the cycle $\sigma$, this means 
we can enumerate the points of the orbit $O$ in such a way that $q_{1}=q,[\gamma] q_{1}=q_{2}, \ldots,[\gamma] q_{k-1}=q_{k}$, and $[\gamma] q_{k}=q_{1}=q$. Therefore, by the construction of the action of $\Pi_{1}(y)$ on $Q_{y}$, for the lift $\widetilde{\gamma}_{1}$ of $\gamma$ to $Q^{\prime}$ such that $\widetilde{\gamma}_{1}(0)=q_{1}$ we have that $\widetilde{\gamma}_{1}(1)=q_{2}$, for the lift $\widetilde{\gamma}_{2}$ of $\gamma$ to $Q^{\prime}$ such that $\widetilde{\gamma}_{2}(0)=q_{2}$ we have that $\widetilde{\gamma}_{1}(1)=q_{3}, \ldots$, and finally for the lift $\widetilde{\gamma}_{k}$ of $\gamma$ to $Q^{\prime}$ such that $\widetilde{\gamma}_{k}(0)=q_{k}$ we have that $\widetilde{\gamma}_{k}(1)=q_{1}=q$.

What do we do in fact is that we take a point $q_{1}=q \in Q_{y}$, then construct the points $q_{2}=[\gamma] q_{1}$, $q_{3}=[\gamma] q_{2}, \ldots$, up to $[\gamma] q_{k}=q_{1}$. Then the set $\left\{q_{1}, q_{2}, \cdots, q_{k}\right\}$ is the orbit $O$ of the point $q$.

It is clear that $\widetilde{\gamma}=\widetilde{\gamma}_{k} \cdot \widetilde{\gamma}_{k-1} \cdots \cdots \widetilde{\gamma}_{1}$, where $\cdot$ is the path composition, is a loop in $\pi_{Q}^{-1}\left(U^{\prime}(x)\right)$ at the point $q_{1}=q, \widetilde{\gamma}$ passes once and only once through each point of $O$, and $\pi_{1}\left(\pi_{E}\right)([\widetilde{\gamma}])=[\gamma]^{k}$. Thus $\widetilde{\gamma}$ is the required loop.

\subsubsection{The index of isolated singular point}

Let $M$ be an oriented two-dimensional manifold. Let $\xi=\left\{\pi_{E}: E \rightarrow M\right\}$ be a locally trivial fiber bundle with standard fiber $F$ and a connected structure Lie group $G$.

Let $Q$ be a branched section of $\xi$ with singularity set $\Sigma$, and $x$ be an isolated point of $\Sigma$. Take a disk neighborhood $U(x)$, and for a point $y \in U^{\prime}(x)$, let $\mathcal{O}_{y}$ be the set of orbits of local monodromy group action on $Q_{y}$. Take an orbit $O \in \mathcal{O}(y)$ and a point $q \in O$. Let $[\gamma]$ be a positive generator of the group $\Pi_{1}(y)$, and $\widetilde{\gamma}$ the loop at $q$ constructed in Proposition 3.6.2.

Let $\psi: \pi_{E}^{-1}(U(x)) \rightarrow U(x) \times F$ be a trivialization of the bundle $\xi$, and $p: \pi_{E}^{-1}(U(x)) \rightarrow F$ be the corresponding projection. Then the element $[p \circ \widetilde{\gamma}] \in \pi_{1}(F)$ is called the index of the branched section $Q$ at the singular point $x$ corresponding to the orbit $O \in \mathcal{O}_{y}$, call it ind $(Q ; y, O)$.

Proposition 3.6.3. a) The index $\operatorname{ind}_{x}(Q ; y, O)$ does not depend on a choice of the loop $\gamma:[0,1] \rightarrow$ $U\left(x^{\prime}\right)$ representing the positive generator of the group $\Pi_{1}(y)$.

b) The index $\operatorname{ind}_{x}(Q ; y, O)$ does not depend on a trivialization.

c) The index $\operatorname{ind}_{x}(Q ; y, O)$ does not depend on a choice of the disk neighborhood $U(x)$, this means that, if $U(x)$ and $V(x)$ are two disk neighborhoods of $x$, and $y \in U(x) \cap V(x)$, then the constructions of $\operatorname{ind}_{x}(Q ; y, O)$ performed for $U(x)$ and for $V(x)$ result in the same element in $\pi_{1}(F)$.

Proof. a) If $\gamma$ and $\mu$ are two representatives of the positive generator of $\Pi_{1}(y)$, then $\gamma$ is homotopic to $\mu$, therefore $\gamma^{k}$ is homotopic to $\mu^{k}$, therefore the lift $\widetilde{\mu}$ of $\mu^{k}$ is homotopic to the lift $\widetilde{\gamma}$ of $\gamma^{k}$, hence $p \widetilde{\gamma}$ is homotopic to $p \widetilde{\mu}$.

b) This is because the gluing functions are homotopic to the identity as the structure group is connected. 
c) This follows directly from the fact that $\Pi_{1}^{U}(y)=\Pi_{1}^{V}(y)$ (see the proof of Proposition 3.6.2), and from a).

Example 3.6.1. Let us consider the trivial bundle $\xi=\left\{\pi_{E}: E=\mathbb{C} \times \mathbb{C}^{*} \rightarrow M=\mathbb{C}\right\}$, where $\mathbb{C}^{*}=$ $\mathbb{C} \backslash\{0\}$ and $\pi_{E}(z, w)=z$. Let us take the subset $Q=\left\{(z, w) \mid z^{2}=w^{3}\right\} \subset E$.

As $\left.\pi_{E}\right|_{Q}: Q \rightarrow M \backslash\{z=0\}$ is a 3 -sheeted covering, we see that $Q$ is a 3-sheeted branched section of the bundle $\xi$.

It is clear that the singularity set of $Q$ is $\Sigma=\{0\}$, so $Q$ has only one singular point $z=0$ and this point is isolated. For the disk neighborhood of the isolated singular point $z=0$ we take the entire $M=\mathbb{C}$.

Let us take $y=1$, then $Q_{y}=\left\{a=(1,1), b=(1, \varepsilon), c=\left(1, \varepsilon^{2}\right)\right\}$, where $\varepsilon=\exp (2 \pi i / 3)$. The loop $\gamma(t)=\exp (2 \pi i t), t \in[0,1]$, represents the positive generator of the group $\Pi(y=1)$, and the lift $\widetilde{\gamma}_{a}$ of $\gamma$ which starts at the point $a=(1,1)$ is given by $\widetilde{\gamma}_{a}(t)=\left(\exp (2 \pi i t), \exp \left(\frac{4}{3} \pi i t\right), t \in[0,1]\right.$. Therefore $[\gamma] a=c$. In the same manner one can prove that $[\gamma] b=a,[\gamma] c=b$.

Thus, the orbit $O$ of the point $a$ is $Q_{y=1}=\{a, b, c\}$, and for a representative of the class $[\widetilde{\gamma}]$ constructed in Proposition 3.2.2 we can take the loop $\widetilde{\gamma}=(\exp (6 \pi i t), \exp (4 \pi i t))$ for $t \in[0,1]$.

Therefore, the loop $p \widetilde{\gamma}:[0,1] \rightarrow \mathbb{C}^{*}$ is given by $\left.p \widetilde{\gamma}=\exp (4 \pi i t)\right)$ for $t \in[0,1]$. Hence

$$
\operatorname{ind}_{0}\left(Q ; y=1, Q_{y=1}\right)=2 \in \mathbb{Z}=\pi_{1}\left(\mathbb{C}^{*}\right) .
$$

Let us consider the finite set of elements of $\pi_{1}(F)$ :

$$
\operatorname{ind}_{x}(Q ; y)=\left\{\operatorname{ind}_{x}(Q ; y, O) \mid O \in \mathcal{O}(y)\right\}
$$

Proposition 3.6.4. The set $\operatorname{ind}_{x}(Q ; y)$ does not depend on $y \in U^{\prime}(x)$.

Proof. Let $y, \bar{y}$ be two points in $U^{\prime}(x)$. Take a curve $\delta:[0,1] \rightarrow U^{\prime}(x)$ such that $\delta(0)=y, \delta(1)=\bar{y}$.

The curve $\delta$ defines the group isomorphism $\psi_{\delta}: \Pi_{1}(y) \rightarrow \Pi_{1}(\bar{y}),[\gamma] \mapsto\left[\delta^{-1} \cdot \gamma \cdot \delta\right]$, where $\delta^{-1}(t)=\delta(1-t)$. Also, $\delta$ defines the bijection $\widetilde{\psi}_{\delta}: Q_{y} \rightarrow Q_{\bar{y}}, q \in Q_{y} \mapsto \bar{q} \in Q_{\bar{y}}$, such that for the lift $\widetilde{\delta}$ of $\delta$ to $Q$ with $\widetilde{\delta}(0)=q$ we have that $\widetilde{\delta}(1)=\bar{q}$. In addition, the bijection $\widetilde{\psi}_{\delta}$ is equivariant is sense that $\widetilde{\psi}_{\delta}([\gamma] q)=\psi_{\delta}([\gamma]) \widetilde{\psi}_{\delta}(q)$.

Therefore $\widetilde{\delta}$ induces a bijection $\alpha_{\delta}: \mathcal{O}(y) \rightarrow \mathcal{O}(\bar{y}), O_{q} \mapsto O_{\widetilde{\psi}_{\delta}(q)}$, where $O_{q}$ is the $\Pi_{1}(y)$-orbit of the point $q \in Q_{y}$ and $O_{\widetilde{\psi}_{\delta}(q)}$ is the $\Pi_{1}(\bar{y})$-orbit of the point $\widetilde{\psi}_{\delta}(q) \in Q_{\bar{y}}$.

Let us prove that the loop $\widetilde{\gamma}$ which passes through the points of the orbit $O_{q} \in \mathcal{O}(y)$ constructed in Proposition 3.6.2 is homotopic in $\pi^{-1}\left(U^{\prime}(x)\right)$ to the corresponding loop of the orbit $O_{\widetilde{\psi}(q)} \in \mathcal{O}(\bar{y})$. 
64 CHAPTER 3. A GENERALIZATION OF GAUSS-BONNET-HOPF-POINCARÉ FORMULA

Let $\gamma$ be a loop at $y \in U^{\prime}(x)$ which represents the positive generator of $\Pi_{1}(y)$. The loop $\widetilde{\gamma}$ constructed in Proposition 3.2.2 is homotopic to the lift of the loop $\gamma^{k}$ starting at a point $q \in Q_{y}$. As the loop $\gamma^{k}$ is freely homotopic to the loop $\bar{\gamma}^{k}$, where $\bar{\gamma}=\delta^{-1} \gamma \delta$, the lift $\widetilde{\gamma}$ is freely homotopic to the lift of $\bar{\gamma}^{k}$ starting at the point $\widetilde{\psi}_{\delta}(q)$, but this lift is in turn homotopic to the loop $\tilde{\bar{\gamma}}$.

Therefore the loops $p \widetilde{\gamma}$ and $p \widetilde{\bar{\gamma}}$ are freely homotopic in $F$, therefore define the same element in $\pi_{1}(F)$. Thus we have that $i n d_{x}(Q ; y, O)=i n d_{x}\left(Q ; \bar{y}, \alpha_{\delta}(O)\right)$ for all $O \in \mathcal{O}(y)$, hence $i n d_{x}(Q ; y)=$ $\operatorname{ind}_{x}(Q ; \bar{y})$.

Corollary 3.6.5. The set ind $_{x}(Q)$ does not depend on the disk neighborhood $U(x)$, this means if $U_{1}(x)$ and $U_{2}(x)$ are disk neighborhoods of an isolated singular point $x \in \Sigma$, and $y_{1} \in U_{1}^{\prime}(x)$ and $y_{2} \in U_{2}^{\prime}(x)$, then the set $\operatorname{ind}_{x}\left(Q ; y_{1}\right)$ constructed via $U_{1}(x)$ and the set $\operatorname{ind}_{x}\left(Q ; y_{2}\right)$ constructed via $U_{2}(x)$ coincide.

Proof. Follows from Proposition 3.7.1

From Proposition 3.6.4 it follows that we can give the following definition.

Definition 3.6.1. Let $Q$ be a branched section of the bundle $\xi$. The index of $Q$ at $x \in M$ is

$$
\operatorname{ind}_{x}(Q)=\operatorname{ind}_{x}(Q ; y)
$$

where $y$ is a point of $U^{\prime}(x)$, where $U(x)$ is a disk neighborhood of $x$.

Let us fix an element $a \in H^{1}(F)$. The index of $Q$ at a point $x$ with respect to $a$ is

$$
\operatorname{ind}_{x}(Q ; a)=\sum_{O \in O(y)} \frac{1}{\# O}\left\langle a, \operatorname{ind}_{x}(Q ; y, O)\right\rangle=\sum_{O \in O(y)} \frac{1}{\# O} \int_{\gamma(Q ; y, O)} \alpha,
$$

where $\alpha \in \Omega^{1}(F)$ represents $a \in H^{1}(F)$ and $\gamma(Q ; y, O)$ represents the class $i n d_{x}(Q ; y, O) \in \pi_{1}(F)$. Example 3.6.2. Let $M$ be a connected compact oriented manifold and let $\omega$ be a symmetric tensor of order $n$ over $M$. In Example 3.5.2 we have constructed a branched section $Q \subset P T M$ determined by the binary differential equation (3.38).

If we consider the covering $q: \mathbb{S}^{1} T M \rightarrow P T M$ given by $q((p, \vec{v}))=[\vec{v}]$, we see that $q \circ \pi: \mathbb{S}^{1} T M \rightarrow$ $M$ is a fiber bundle and $q^{-1}(Q)$ is a $2 n$-sheeted branched section of the bundle $\mathbb{S}^{1} T M \rightarrow M$. Let $p \in \Sigma$ be a singular point, $U^{\prime}(p)$ be a neighborhood disk of $p$, and $\mathcal{O}_{p}=\left\{O_{1}, \cdots, O_{r}\right\}$ the set of the orbits of the action of $\pi_{1}\left(U^{\prime}(p)\right)$ on $\pi^{-1}(p)$. From the equation (3.43) it follows that the index of $q^{-1}(Q)$ at the singular point $p \in \Sigma$ with respect the cohomology class $a=\left[\frac{1}{2 \pi} d \theta\right] \in H^{1}\left(\mathbb{S}^{1}\right)$, where $d \theta$ is the angular form on $\mathbb{S}^{1}$ is given by

$$
\operatorname{ind}_{p}(Q ; a)=\sum_{i=1}^{r} \frac{1}{2 \pi k_{i}} \int_{\gamma_{i}} d \theta,
$$


where $k_{i}$ is the number of elements of the orbit $O_{i}$, and $\gamma_{i}$ is the index of the point $p$ corresponding to the orbit $O_{i}$. Let us choose a frame $\left(e_{1}, e_{2}\right)$ along the curve $\gamma$, and consider a unit vector field $X(t), 0 \leq t \leq 1$ such that $\omega_{\gamma(t)}(X(t))$ around the curve $\gamma: I \rightarrow U^{\prime}(p)$. If $\tilde{\theta}$ is the angle between $e_{1}$ and $X(0)$, we obtain that the index of $Q$ at the point $p$ with respect to the form $a$ can be also calculated in terms of this angle by the formula

$$
\operatorname{ind}_{p}\left(Q, O_{i}, a\right)=\frac{\tilde{\theta}\left(2 k_{i}\right)-\tilde{\theta}(0)}{2 \pi k_{i}} .
$$

Note that if the action of $\pi_{1}\left(U^{\prime}(p)\right)$ on $\pi^{-1}(p)$ is transitive, then the equation (3.44) reduces to the following

$$
\operatorname{ind}_{p}(Q ; a)=\frac{1}{4 \pi n} \int_{\gamma} d \theta
$$

where $\gamma$ is the index of $p$ in $\pi^{-1}(p)$, and it is also true that

$$
i n d_{p}\left(Q, \pi^{-1}(p), a\right)=\frac{\tilde{\theta}\left(2 k_{i}\right)-\tilde{\theta}(0)}{4 n \pi} .
$$

The equation (3.47) coincides with the index of a binary differential $n$-form given in [28].

Now, we note that the index of $Q$ at a singular point $x$ seen as a singularity of the bundle $\pi$ : $P T M \rightarrow M$ is twice the index of the same point as a singular point of the bundle $\pi \circ q: \mathbb{S}^{1} T M \rightarrow M$.

Remark 3.6.1. This construction can be used to calculate an index of singular points of singular distributions over a two dimensional manifold $M$. In [20], (pages 218-223), the author gives another constructions of indexes of singular points of 1-dimensional singular distributions and branched sections of two sheets defined by such a distributions.

\subsection{Resolution of a branched section}

Let $M$ be a two-dimensional oriented manifold, and $\xi=\left\{\pi_{E}: E \rightarrow M\right\}$ be a fiber bundle. Let $\Sigma$ be a discrete subset of the manifold $M$.

Definition 3.7.1. Let $Q$ be an $n$-sheeted branched section of the bundle $\xi$ with singularity set $\Sigma$, $M^{\prime}=M \backslash \Sigma, E^{\prime}=\pi^{-1}\left(M^{\prime}\right)$, and $Q^{\prime}=Q \cap E^{\prime}$. A resolution of $Q$ is a map $\iota: S \rightarrow E$, where $S$ is an oriented two-dimensional manifold with boundary, such that

1. $\iota(S)=Q$;

2. $\pi=\pi_{E} \circ \iota: S \rightarrow M$ is surjective; 
3. the map $\iota$ is a diffeomorphism of $S^{\prime}=S \backslash \partial S$ onto $Q^{\prime}$.

In case $M$ is compact, we assume $S$ to be compact, too.

Remark 3.7.1. From Definition 3.7.1 it follows that $\pi_{E}(Q)=M$ and $\pi_{E}(\partial S)=\Sigma$.

Example 3.7.1. Let $M=\mathbb{R}^{2}, E=\mathbb{P} T \mathbb{R}^{2}$ and a branched section is the solution of the differential equation $x y d x^{2}-\left(x^{2}-y^{2}\right) d x d y-x y d y^{2}=0$. As the discriminant of this equation is $\left(x^{2}-y^{2}\right)^{2}-$ $4(x y)^{2}=\left(x^{2}+y^{2}\right)^{2}$, this differential equation is a binary differential equation (see Example 3.5.2). This differential equation is represented in the form $(x d x+y d y)(y d x-x d y)=0$, therefore its solution $Q$ consists of two 1-dimensional distributions $L_{1}$ and $L_{2}$ on $\mathbb{R}^{2}$ given respectively by the equations $x d x+y d y=0$ and $y d x-x d y=0$. One can easily see that these equations determine sections with singularities $s_{1}$ and $s_{2}$ of the bundle $E$, which admit resolutions (see 3.1), call them $S_{1}$ and $S_{2}$, so the manifold $S_{1} \sqcup S_{2}$ is a resolution of the branched section $Q$.

Example 3.7.2. Let $M=\mathbb{R}^{2}, E=P T \mathbb{R}^{2}$ and the branched section $Q$ is the solution of the binary differential equation

$$
y d x^{2}-2 x d x d y-y d y^{2}=0 .
$$

The discriminant of equation (3.48) is $4\left(x^{2}+y^{2}\right)$, therefore this equation has two real roots for all $(x, y)$ different from the origin, and at the origin all the coefficients vanish. That is why, equation (3.48) is a binary differential equation (see Example 3.5.2).

The standard coordinates $(x, y)$ on $\mathbb{R}^{2}$ induce a trivialization of the bundle $\pi_{E}=E=P T \mathbb{R}^{2} \rightarrow$ $M=\mathbb{R}^{2}$, namely for the one-dimensional subspace $l \in P T_{(x, y)} \mathbb{R}^{2}$ spanned by a vector $p \partial_{x}+q \partial_{y}$, we assign the point $(x, y,[p: q]) \in \mathbb{R}^{2} \times \mathbb{R} P^{1}$. Thus, $P T \mathbb{R}^{2} \cong \mathbb{R}^{2} \times \mathbb{R} P^{1}$, and

$$
Q=\left\{(x, y,[p: q]) \in \mathbb{R}^{2} \times \mathbb{R} P^{1} \mid y p^{2}-2 x p q-y q^{2}=0\right\}
$$

In this case

$$
\Sigma=(0,0), \quad Q^{\prime}=\left\{(x, y,[p: q]) \in Q \mid x^{2}+y^{2}>0\right\}, \quad M^{\prime}=\mathbb{R}^{2} \backslash\{(0,0)\}
$$

The projection $\pi_{P T \mathbb{R}^{2}}: P T \mathbb{R}^{2} \rightarrow \mathbb{R}^{2}$ restricted to $Q^{\prime}$ is a trivial (as a fiber bundle) double covering of $M^{\prime}$. Indeed, take the following open sets $U_{1}$ and $U_{2}$ :

$$
U_{1}=M^{\prime} \backslash(-\infty, 0) \times\{0\} \text { and } U_{2}=M^{\prime} \backslash(0, \infty) \times\{0\}
$$

It is clear that $M^{\prime}=U_{1} \cup U_{2}$. Also, at the points of $U_{1}$ we have $x+\sqrt{x^{2}+y^{2}}>0$, and at the points of $U_{2}$ we have $x-\sqrt{x^{2}+y^{2}}>0$. 
Now let us take two sections of the bundle $\pi_{P T \mathbb{R}^{2}}: P T \mathbb{R}^{2} \rightarrow \mathbb{R}^{2}$ defined on $M^{\prime}$ :

$$
s_{1}:(x, y) \mapsto \begin{cases}\left(x, y,\left[x+\sqrt{x^{2}+y^{2}}: y\right]\right), & (x, y) \in U_{1}, \\ \left(x, y,\left[-y: x-\sqrt{x^{2}+y^{2}}\right]\right), & (x, y) \in U_{2},\end{cases}
$$

and

$$
s_{2}:(x, y) \mapsto \begin{cases}\left(x, y,\left[-y: x+\sqrt{x^{2}+y^{2}}\right]\right), & (x, y) \in U_{1}, \\ \left(x, y,\left[x-\sqrt{x^{2}+y^{2}}: y\right]\right), & (x, y) \in U_{2},\end{cases}
$$

Note that over $U_{1} \cap U_{2}$ there holds

$$
\left[x+\sqrt{x^{2}+y^{2}}: y\right]=\left[-y: x-\sqrt{x^{2}+y^{2}}\right] \text { and }\left[-y: x+\sqrt{x^{2}+y^{2}}\right]=\left[x-\sqrt{x^{2}+y^{2}}: y\right],
$$

therefore the sections $s_{1}$ and $s_{2}$ are well defined. One can easily prove that $s_{i}\left(M^{\prime}\right) \subset Q^{\prime}, i=1,2$, and $s_{1}\left(M^{\prime}\right) \cap s_{2}\left(M^{\prime}\right)=\emptyset$. Therefore $Q^{\prime}$ is a trivial double covering of $M^{\prime}$.

Now let us construct a resolution of the branched section $Q$. Recall that $\mathbb{S}^{1}=\left\{(u, v) \mid u^{2}+v^{2}=1\right\}$. Let us take the diffeomorphism

$$
f: \mathbb{S}^{1} \rightarrow \mathbb{R} P^{1},(u, v) \mapsto \begin{cases}{[u+1: v],} & u>-1, \\ {[-v: u-1],} & u<1,\end{cases}
$$

then the diffeomorphism $f$ "rotated" at the angle $\pi / 2$ gives the diffeomorphism,

$$
g: \mathbb{S}^{1} \rightarrow \mathbb{R} P^{1},(u, v) \mapsto\left\{\begin{array}{l}
{[-v: u+1], \quad u>-1,} \\
{[u-1: v], \quad u<1 .}
\end{array}\right.
$$

We take $S_{1}=S_{2}=\mathbb{R}_{+} \times \mathbb{S}^{1}=[0, \infty) \times \mathbb{S}^{1}$, and $S_{1}^{\prime}=S_{2}^{\prime}=(0, \infty) \times \mathbb{S}^{1}$. We set $S=S_{1} \sqcup S_{2}$, then $S^{\prime}=S_{1}^{\prime} \sqcup S_{2}^{\prime}$. Then $\iota: S \rightarrow \mathbb{R}^{2} \times \mathbb{R} P^{1}$ is given by

$$
\iota\left|S_{1}(r,(u, v))=(r u, r v, f(u, v)), \quad \iota\right|_{S_{2}}(r,(u, v))=(r u, r v, g(u, v)) .
$$

One can easy see that $\iota_{S_{i}^{\prime}}: S_{i}^{\prime} \rightarrow Q_{i}^{\prime}, i=1,2$ is a diffeomorphism. For example, any point $(x, y,[p: q]) \in V_{11}$, is the image of the point $(r,(u, v))$ under the map $\left.\iota\right|_{S_{1}}$, where

$$
u=\frac{x}{\sqrt{x^{2}+y^{2}}}, \quad v=\frac{y}{\sqrt{x^{2}+y^{2}}}, \quad r=\sqrt{x^{2}+y^{2}} .
$$

Example 3.7.3. As a generalization of Examples 3.3.2 and 3.3.3 one can take $n$ sections with singularities (see 3.1) of a bundle $\xi=\pi_{E}: E \rightarrow M$ call them $s_{i}, i=\overline{1, n}$ which have the same set of singularities $\Sigma$. These sections define a branched section $Q$ of the bundle $\xi: Q=\left\{s_{i}(x) \mid x \in M \backslash \Sigma\right\}$. If $S_{i}$ is a resolution of $s_{i}$, then $S=\sqcup S_{i}$ is a resolution of $Q$. 
Example 3.7.4. Let us present an example of branched section, where the covering $\left.\pi_{Q}\right|_{Q^{\prime}}: Q^{\prime} \rightarrow M^{\prime}$ is not trivial. Take $M=\mathbb{R}^{2}=\mathbb{C}, E=\mathbb{S}^{1}(\mathbb{C})=\mathbb{C} \times \mathbb{S}^{1}$, the bundle of unit vectors over $M$, and let

$$
Q=\left\{(z, w) \in \mathbb{C} \times \mathbb{S}^{1}|| z \mid w^{2}=z\right\} .
$$

Then $M^{\prime}=\mathbb{C} \backslash\{0\}, Q^{\prime}=\left\{(z, w)\left|w^{2}=z /\right| z \mid\right\}$, and it is well known that $\left.\pi_{Q}\right|_{Q^{\prime}}: Q^{\prime} \rightarrow M^{\prime}$ is a non trivial double covering. Now let us take

$$
S=[0, \infty) \times \mathbb{S}^{1}, \text { and } \iota: S \rightarrow E, \quad\left(r, e^{i \varphi}\right) \mapsto\left(r e^{2 i \varphi}, e^{i \varphi}\right)
$$

Then $S^{\prime}=(0, \infty) \times \mathbb{S}^{1}$, and it is clear that the properties (1)-(3) of Definition 3.7.1 hold true for $\iota$. Example 3.7.5. Let us present another example of branched section, where the covering $\left.\pi_{Q}\right|_{Q^{\prime}}: Q^{\prime} \rightarrow$ $M^{\prime}$ is not trivial. Take $M=\mathbb{R}^{2}=\mathbb{C}, E=P T \mathbb{R}^{2}=\mathbb{R}^{2} \times \mathbb{R} P^{1}=\mathbb{C} \times \mathbb{R} P^{1}$, and let

$$
Q=\left\{\left(z,[w]|| w \mid=1 \text { and }|z|^{2} w^{4}=z^{2}\right\}\right.
$$

Then $M^{\prime}=\mathbb{C} \backslash\{0\}, Q^{\prime}=\left\{\left.(z, w)\left|w^{4}=z^{2} /\right| z\right|^{2}\right\}$, and it is clear that $\left.\pi_{Q}\right|_{Q^{\prime}}: Q^{\prime} \rightarrow M^{\prime}$ is a non trivial double covering. Now let us take

$$
S=[0, \infty) \times \mathbb{R} P^{1}, \text { and } \iota: S \rightarrow E, \quad(r,[w]) \mapsto\left(r w^{2},[w]\right),
$$

where $|w|=1$. Then $S^{\prime}=(0, \infty)$, and it is clear that the properties (1)-(3) of Definition 3.3.1 hold true for $\iota$.

Remark 3.7.2. In Examples 3.7.4-3.7.5, for each $x \in M$, the set $S_{x}$ is a discrete set if $x \in M \backslash \Sigma$, or is diffeomorphic to the circle $\mathbb{S}^{1}$ if $x \in \Sigma$.

Now let us consider a point $x \in \Sigma$. Then, according to Definition 3.3.1, $S_{x}=\pi^{-1}(x)$ consists of the connected components of the boundary $\partial S$. Let us denote by $C\left(S_{x}\right)$ the set of connected components of $S_{x}$. As $S_{x}$ is compact, the set $C\left(S_{x}\right)$ is finite, and each element of this set is diffeomorphic to the circle $\mathbb{S}^{1}$.

Proposition 3.7.1. Let $C$ be a connected component of the boundary of $S$. Then there exists a neighborhood $N(C)$ of $C$ and a diffeomorphism $f_{C}: N(C) \rightarrow \mathbb{S}^{1} \times[0,1]$ such that $f_{C}(C)=\mathbb{S}^{1} \times\{0\}$ and $U(x)=\pi\left(N(C)\right.$ is a disk neighborhood of $x$. For each $y \in U^{\prime}(x)$, the set of orbits $\mathcal{O}_{y}$ consists of only one element. In this cases the curve $\widehat{\gamma}$ corresponding to the orbit by Proposition 3.2.2 is a generator of the group $\pi_{1}(N(C)) \cong \mathbb{Z}$.

Proof. Indeed, $N(C) \backslash C$ is homeomorphic to a ring and $U^{\prime}(x)$ is homeomorphic to a ring as well. The map $N(C) \backslash C \rightarrow U^{\prime}(x)$ induced by $\pi$ is a $n$-fold covering therefore $\pi_{*}: \pi_{1}(N(C)) \cong \mathbb{Z} \rightarrow \pi_{1}\left(U^{\prime}(x)\right)$ has the form $m \rightarrow k m$. At the same time $\pi_{*}([\widetilde{\gamma}])=\gamma^{k}$, thus $[\widetilde{\gamma}]$ is a generator of the group $\pi_{1}(N(C))$. 
Corollary 3.7.2. The curve $\widetilde{\gamma}$ is homotopic in $N(C) \subset S$ to the curve $C \subset E_{x}$. Therefore the curve $C$ represents $\operatorname{ind}_{x}(Q, O)$.

\subsection{Connection and the Gauss-Bonnet theorem}

Let $\xi=\left(\pi_{E}: E \rightarrow M\right)$ be a locally trivial fiber bundle with standard fiber $F$ and structure group $G$. Assume that $G$ is a connected Lie group.

Let $\left(U, \psi: \pi^{-1}(U) \rightarrow U \times F\right)$ be a chart of the atlas of $\xi$. Let

$$
\eta=p_{F} \circ \psi: \pi^{-1}(U) \rightarrow F
$$

where $p_{F}: U \times F \rightarrow F$ is the canonical projection onto $F$. For each $x \in U$ the map $\eta$ restricted to $F_{x}=\pi^{-1}(x)$ induces a diffeomorphism $\eta_{x}: F_{x} \rightarrow F$, and let $i_{x}: F \rightarrow F_{x}$ be the inverse of $\eta_{x}$. Note that if we take another chart $\left(U^{\prime}, \psi^{\prime}: \pi^{-1}\left(U^{\prime}\right) \rightarrow U^{\prime} \times F\right)$, and $\eta^{\prime}: \pi^{-1}\left(U^{\prime}\right) \rightarrow F$ is the corresponding map, then on $\pi^{-1}\left(U \cap U^{\prime}\right)$ we have that

$$
\psi^{\prime} \circ \psi^{-1}:\left(U \cap U^{\prime}\right) \times F \rightarrow\left(U \cap U^{\prime}\right) \times F, \quad(x, y) \mapsto(x, g(x) y)
$$

where $g: U \cap U^{\prime} \rightarrow G$ is the gluing map of the charts. Now, for any $x \in U \cap U^{\prime}$, we have $\eta_{x}^{\prime} \circ \eta_{x}^{-1}(y)=g(x) y$, and, as $G$ is connected, $\eta_{x}^{\prime} \circ \eta_{x}^{-1}: F \rightarrow F$ is homotopic to the identity map. This means that for any $x \in M$ we have well defined isomorphisms of the homotopy and (co)homology groups:

$$
\begin{aligned}
& \pi_{*}\left(\eta_{x}\right): \pi_{*}\left(F_{x}\right) \rightarrow \pi_{*}(F), \\
& H_{*}\left(\eta_{x}\right): H_{*}\left(F_{x}\right) \rightarrow H_{*}(F), \quad H^{*}\left(\eta_{x}\right): H^{*}(F) \rightarrow H^{*}\left(F_{x}\right),
\end{aligned}
$$

which do not depend on the chart.

In Section 3.2, for a locally trivial bundle with standard fiber $F$ and structure Lie group $G$, we have proved the following statement ( see Theorem 3.2.7)

Proposition 3.8.1. Let $a \in H^{1}(F)$ and $H$ be a connection in E. There exists a 1 -form $\alpha \in \Omega^{1}(E)$ such that

1. $\left.\alpha\right|_{H}=0$;

2. for each $x \in M, d i_{x}^{*} \alpha=0$ and $\left[i_{x}^{*} \alpha\right]=H^{1}\left(\eta_{x}\right) a$. 
The decomposition $T E=H \oplus V$ gives a bicomplex representation of the complex $\Omega(E)$, then the form $\alpha$ lies in $\Omega^{(0,1)}(E)$ and $d \alpha=\theta_{(1,1)}+\theta_{(2,0)}$, where $\theta_{(1,1)} \in \Omega^{(1,1)}(E)$ and $\theta_{(2,0)} \in \Omega^{(2,0)}(E)$, and

$$
\theta_{(1,1)}(X, Y)=\left(L_{X} \alpha\right)(Y), \quad \theta_{(2,0)}=\widetilde{\alpha}(\Omega)
$$

where $L_{X}$ is the Lie derivative with respect to the vector field $X$, and $\Omega$ is the curvature form of the connection $H$ (for details see 3.2.4).

Now let $Q$ be a branched section of the bundle $\xi$ which admits a resolution $\iota: S \rightarrow E$ (see Definition 3.7.1). Let us fix an element $a \in H^{1}(F)$, and let $\alpha \in \Omega^{1}(E)$ be the corresponding 1-form (see Proposition 3.8.1). Then, by the Stokes theorem we have

$$
\int_{\partial S} \iota^{*} \alpha=\int_{S} \iota^{*} d \alpha
$$

By Remark 3.7.1 we have that $\pi_{E}(\partial S)=\Sigma$. For $x \in \Sigma$, let $C\left(S_{x}\right)$ be the set of connected components of $\pi_{E}^{-1}(x)$.

From Corollary 3.7.2, it follows that, for $C \in C\left(S_{x}\right)$, we have

$$
\int_{C} \alpha=\int_{\gamma(Q ; y, O(C)} i_{x}^{*} \alpha
$$

where $\gamma(Q ; y, O(C))$ represents the class $\operatorname{ind}_{x}(Q ; y, O(C)) \in \pi_{1}(F)$, and $O(C)$ is the orbit of the local monodromy group corresponding to $C$. Therefore, from (3.43) we have that

$$
\operatorname{ind}_{x}(Q ; a)=\sum_{C \in C\left(S_{x}\right)} \frac{1}{\# O(C)} \int_{C} \alpha .
$$

If all the orbits of the local monodromy group corresponding to the components $C \in C\left(S_{x}\right)$ have the same number of elements $N(x)$, then

$$
\int_{\partial S} \iota^{*} \alpha=\sum_{x \in \Sigma} \sum_{C \in C\left(S_{x}\right)} \int_{C} \alpha=\sum_{x \in \Sigma} N(x) \operatorname{ind}_{x}(Q ; a)
$$

Thus we get the following theorem

Theorem 3.8.2 (Gauss-Bonnet-Hopf-Poincaré formula for branched sections). If, for any $x \in \Sigma$, all the orbits of the local monodromy group corresponding to the components $C \in C\left(S_{x}\right)$ have the same number of elements $N(x)$, then

$$
\int_{S} \iota^{*} \theta_{(1,1)}+\iota^{*} \theta_{(2,0)}=\sum_{x \in \pi(\partial S)} N(x) i n d_{x}(Q) .
$$




\section{Bibliography}

[1] A. Hatcher Algebraic Topology, Cambridge University Press, 2002.

[2] A. Mukherjee, Differential Topology, Birkauser Basel, 2015.

[3] B. Dubrovin, A. Fomenko., S. Novikov. Modern Geometry, Introduction to Homology Theory, part III, Springer, 1990.

[4] B. Dubrovin, A. Fomenko., S. Novikov. Modern Geometry-Methods and Applications, part II, Springer.

[5] B. N. Shapukov, Projective bundles and projective connections, Russian Mathematics, Izvestia VUZ Mathematika, 1995.

[6] C. H. Taubes, Differential Geometry: Bundles, Connections, Metrics and Curvature, Oxford Graduate Texts in Mathematics 2011

[7] C.J, Isham Modern Differential Geometry for Physicists, second edition, Word Scientific, 1998.

[8] D. Bell, The Gauss-Bonnet Theorem for Vector Bundles, J. Geom. 85, 2006, 15-21.

[9] D. V. Alekseevivskij, A.M. Vinogradov, V.V. Lichagyn Geometry I: Basic ideas and concepts of differential geometry, Springer, 1988.

[10] F. A. Arias. and M. A. Malakhaltsev, A generalización of the Gauss-Bonnet and Hopf-Poincaré theorems, ArXiv:1510.01395 [MathDG] 5 Oct 2015, 2015.

[11] F. A. Arias. and M. A. Malakhaltsev, A generalización of the Gauss-Bonnet and Hopf-Poincaré theorems. Part II.

[12] F. A. Arias, J. Arteaga, and M. A. Malakhaltsev 3-webs with singularities, Lobachevskii Journal of Mathematics, 2016. 
[13] G. E. Bredon, Topology and Geometry, Springer, 1997.

[14] I. Kolar, P. Michor. P, J. Slovak Natural operations in differential geometry. Berlin: SpringerVerlag, 1993.

[15] I. Madsen, J. Tornehave, From calculus to cohomology, Cmbridge University press, 1997

[16] J. F, Davis J. P, Kirk, Lecture Notes in Algebraic Topology, Department of Mathematics, Indiana University, 1991.

[17] J, Munkres, Topology, Prentice Hall, 2000

[18] J. Rotman, An Introduction to Algebraic Topology, Springer, 1988.

[19] J W Bruce and F Tari, emphOn binary differential equations, http://dx.doi.org/10.1088/09517715/8/2/008, Nonlinearity, 1995.

[20] M. Spivak, A comprehensive introduction to differential geometry. Vol. 1-5. 3rd ed. with corrections, 3rd ed. with corrections, Houston, TX: Publish or Perish, 1999.

[21] N, Steenrod, The Topology of Fiber Bundles, Princeton University Press, 1952.

[22] P. W. Michor., Topics in differential geometry, Springer-Verlag 2000

[23] R. W Sharpe, Differential geometry, Springer 2000.

[24] S. Kobayashi and K. Nomizu, Foundations of differential Geometry, Volume I, Interscience publishers, 1963.

[25] S. Kobayashi and K. Nomizu, Foundations of differential Geometry, Volume I, Interscience publishers, 1969.

[26] S. Helgason Differential Geometry, Lie Groups, Symmetric Spaces, Department of Mathematics Massachusetts Institute of Technology Cambridge, Massachusetts.

[27] S.S. Chern, A Simple Intrinsic Proof of the Gauss-Bonnet Formula for Closed Riemannian Manifolds, Ann. Math. 45 (4), 1944, 747-752.

[28] T. Fufuki, J. J. Nuño-Ballesteros, Isolated points of binary differential equations of degree $n$, Mathematics subject classification, 2010. 